\title{
An alternative to dark matter?
}

\section{A cosmological model of galaxy rotation using cosmological gravity \\ force, $F_{\Lambda}(\mathrm{A} \Lambda \Omega)$}

Jean Perron

Department of applied sciences, UQAC, 555 boul Université, Chicoutimi, Qc, Canada, G7H2B1

Correspondence should be addressed to Jean Perron; jean_perron@uqac.ca 


\begin{abstract}
A cosmological model was developed using the equation of state of photon gas, as well as cosmic time. The primary objective of this model is to see if determining the observed rotation speed of galactic matter is possible, without using dark matter (halo) as a parameter. To do so, a numerical application of the evolution of variables in accordance with cosmic time was developed to determine precise, realistic values for a number of cosmological parameters, such as energy, cosmological constant $\Lambda$, curvature of space $\mathrm{k}$, energy density $\rho_{\Lambda e}$, etc. Several starting assumptions were put forth in order to solve these equations. The current version of the model partially explains several of the observed phenomena that raise questions. Numerical application of the model has yielded the following results, among others: Initially, during the Planck era, at the very beginning of Planck time, $t_{p}$, the universe contained a single photon at Planck temperature and Planck energy in the Planck volume. During the photon inflation phase (before characteristic time $\sim 10^{-9}[s]$ ), the number of photons increased at each unit of Planck time and geometrical progression $\sim \mathrm{n}^{3}$, where $\mathrm{n}$ is the quotient of cosmic time over Planck time $\left(\mathrm{t} / \mathrm{t}_{\mathrm{p}}\right)$. Then, the primordial number of photons reached a maximum of $\sim 10^{89}$, where it remained constant. Such geometric growth in the number of photons can bring a solution to the horizon problem through photonphoton exchange and a photon energy volume that is in phase with that of the universe. The age of the universe in cosmic time that is in line with positive energy conservation (in terms of conventional thermodynamics) and the creation of proton, neutron, electron, and neutrino masses, is $\sim 76$ [Gy]. The predicted total mass ( $\mathrm{p}, \mathrm{n}$, e and $v$ ), based on the Maxwell-Juttner relativistic statistical distribution, is $\sim 7 \times 10^{50}[\mathrm{~kg}]$. The predicted cosmic neutrino mass is $\leq 8.69 \times 10^{-32}[\mathrm{~kg}]$ $\left(\leq 48.7\left[\mathrm{keVc}^{-2}\right]\right)$ if based on observations of SN1987A. The temperature variation of the cosmic microwave background (CMB), as measured by Planck, can be said to be partially due to energy
\end{abstract}


variations in the universe $(\Delta \mathrm{E} / \mathrm{E})$ during the primordial baryon synthesis (energy jump from the creation of protons and neutrons), through a process called baryon asymmetry and the MaxwellJuttner relativistic distribution. In this model, what is usually referred to as dark energy actually corresponds to the energy of the universe that has not been converted to mass, and which acts on the mass created by the mass-energy equivalence principle and the cosmological gravity field, $\mathrm{F}_{\Lambda}$, associated with the cosmological constant, which is high during primordial formation of the galaxies $(<1[\mathrm{~Gy}])$. A look at the Casimir effect makes it possible to estimate a minimum Casimir pressure and thus determine our possible relative position in the universe at cosmic time 0,1813 $\left(\mathrm{t}_{0} / \mathrm{t}_{\Omega}=13,8[G y] / 76,1[G y]\right)$. Therefore, from the observed age of $13,8[\mathrm{~Gy}]$, we can derive a possible cosmic age of $\sim 76,1$ [Gy]. That energy of the universe, when taken into consideration during the formation of the first galaxies $(<1[\mathrm{~Gy}])$, provides a relatively adequate explanation of the non-Keplerian rotation of galactic masses. Indeed, such residual, non-baryonic energy, when considered in Newton's gravity equation, adds the term $\mathrm{F}_{\Lambda}(\mathrm{r})$, which can partially explain, without recourse to dark matter, the rotations of some galaxies, such as M33, UGC12591, UGC2885, NGC3198, NGC253, DDO161, UDG44, the MW and the Coma cluster. Today, that cosmological gravity force is in the order of $10^{26}$ times smaller than the conventional gravity force. The model predicts an acceleration of the mass in the universe $(q \sim-0,986)$; the energy associated with curvature $E_{k}$ is the driving force behind the expansion of the universe, rather than the energy associated with the cosmological constant $E_{\Lambda}$. An equation to determine expansion is obtained using the energy form of the Friedmann equation relative to Planck power and cosmic time or Planck force acting at the frontier of the universe. Finally, the model partly explains the value a 0 of the MOND theory. Indeed, $\mathrm{a}_{0}$ is not a true constant, but depends on the cosmological constant at the time the great structures were formed ( 1 [Gy]), as well as an adjustment of the typical mass 
and dimension of those great structures, such as galaxies. The constant $\mathrm{a}_{0}$ is a different expression of the cosmological gravity force as expressed by the cosmological constant, $\Lambda$, acting through the mass-energy equivalent during the formation of the structures. It does not put in question the value of $\mathrm{G}$.

Keywords: cosmological parameters numerical values, cosmology early universe, galaxies kinematic and dynamic, galaxies Coma cluster, galaxies evolution. 
Table of contents

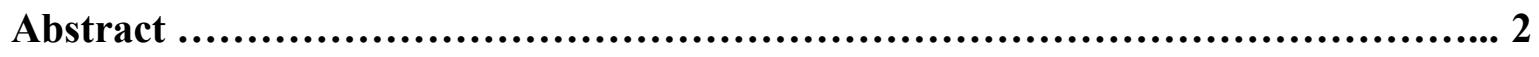

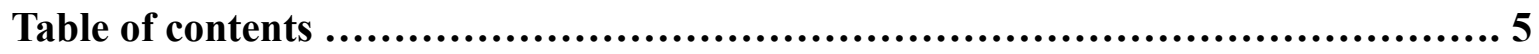

1- Introduction: Formulation of the model, initial concept $\ldots . \ldots \ldots \ldots \ldots \ldots \ldots \ldots \ldots . . . . .7$

2- Equation of state for the temperature, pressure, volume ................... 8

3- State equation, evolution of photon gas, temperature, volume and pressure .. 10

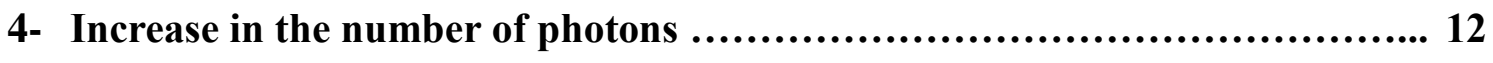

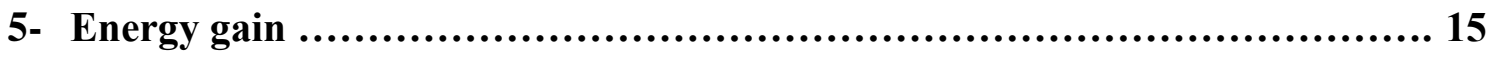

6- A possible solution to the horizon problem? .................................. 20

7- Early baryogenesis (protons, neutrons) and leptons (electrons, neutrinos) .... 24

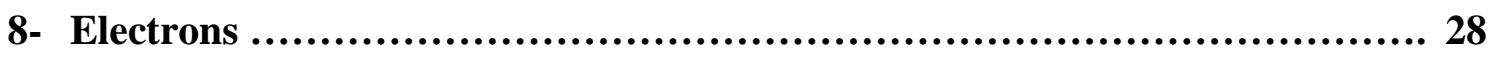

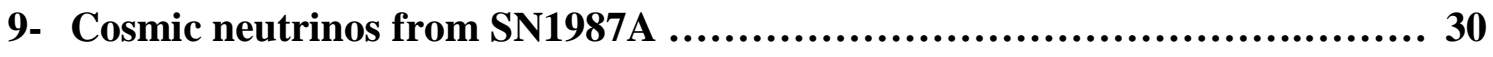

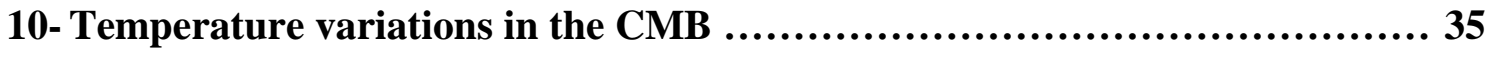

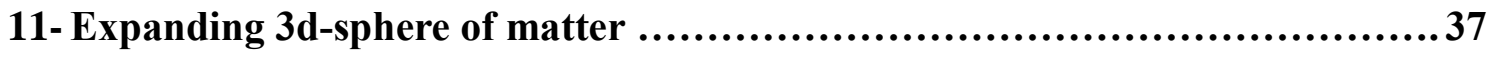

12- Pressure in the CMB and the Casimir effect: A possible age of the universe .. 39

13- A possible baryonic matter-free zone caused by proton and electron time lags 52

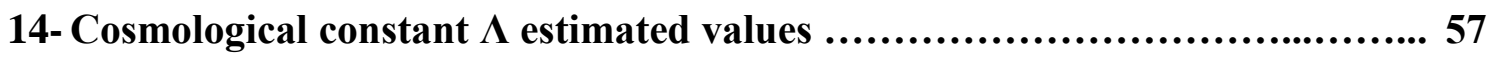

15- The energy form of the Friedmann equation $. . \ldots \ldots \ldots \ldots \ldots \ldots \ldots \ldots \ldots \ldots \ldots \ldots . . . . \ldots 1$

16- Some comparison with data from the $\Lambda$ CDM model $\ldots \ldots \ldots \ldots \ldots \ldots \ldots \ldots \ldots \ldots . . \ldots 3$

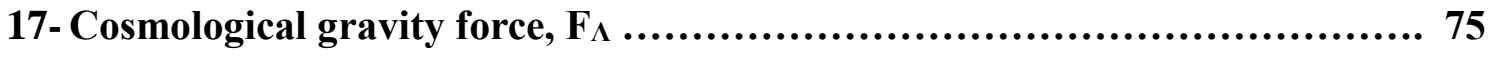

18- Attractive cosmological gravity, $F_{\Lambda}$, and galaxy rotation (simplified model) .. 83

19- Mass rotation equation and tangential velocity $\ldots \ldots \ldots \ldots \ldots \ldots \ldots \ldots \ldots \ldots \ldots \ldots \ldots . . . .68$ 


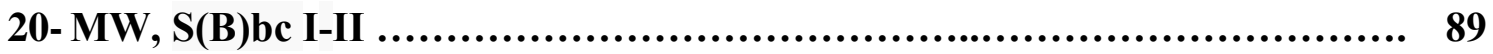

21- M33 (SA(s)cd) (of the triangle) ..................................... 93

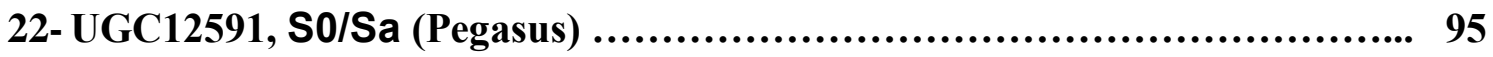

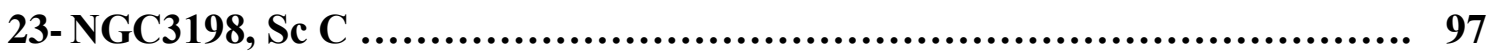

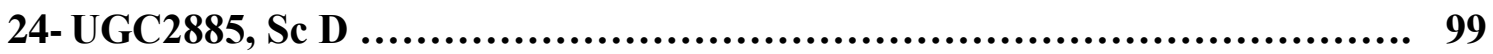

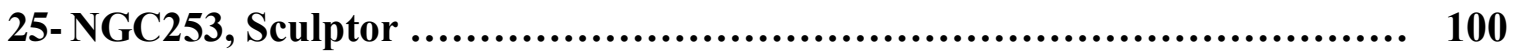

26- Irregular dwarf galaxy DDO161 ....................................... 102

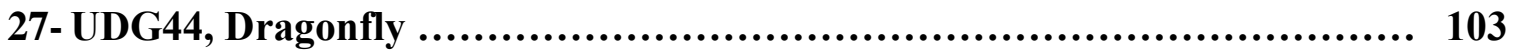

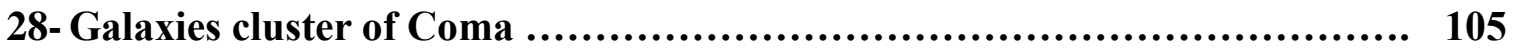

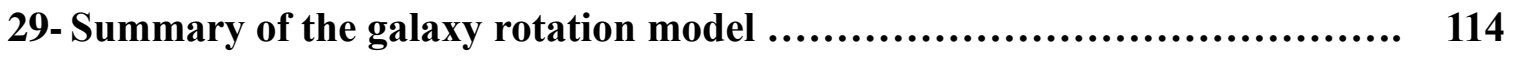

30- Relative position of galaxies .............................................. 116

31- MOND theory and cosmological constant ................................. 118

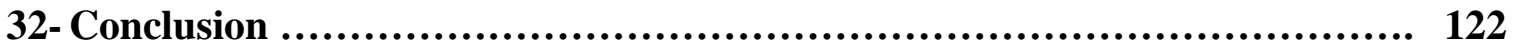

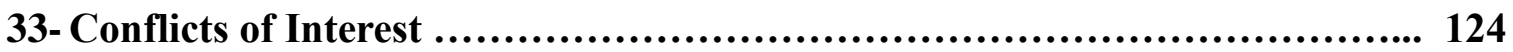

34- Funding Statement .................................................... 124

35- Acknowledgement ........................................................ 124

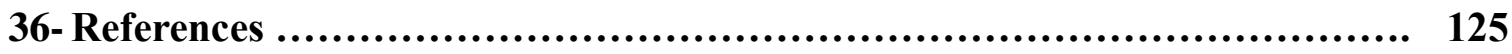




\section{Introduction: Formulation of the model, initial concept}

Cosmology fascinates. Sky-watching has forever been an integral part of human experience. Unfortunately, we do not have all the data we need to fully understand the distant past, what we call the beginning of all things, until today, or even until the so-called end. Nevertheless, we do have numerous findings that allow us to reconstruct, to a greater or lesser extent, the sequence of events from the very beginning, if at all possible, using the laws of physics. The model herein is based on the following key premises, some of which are tested, while others are purely speculative.

The following are the key premises of the model:

- The macroscopic laws of physics applied after the Planck era;

- At the beginning $\left(1 t_{p}\right)$, all of the energy in the universe was electromagnetic (photons); the conventional photon gas equation of state applies;

- Variations in the entropy of the universe, towards what would be considered outside the universe, is zero;

- All infinitesimal variations of $\mathrm{dr}, \mathrm{dT}, \mathrm{dP}, \mathrm{dV}$ and similar variables are to be considered and maintained in the elaboration of differentials equations given the large and small quantities involved in the equation terms (e.g. $\mathrm{t}_{\mathrm{p}} \sim 10^{-43}[\mathrm{~s}], \mathrm{T}_{\mathrm{p}} \sim 10^{32}[\mathrm{~K}]$ );

- The law of conservation of energy applies to universe-size scales;

- The cosmological principle is not necessarily adhered to;

- The Hubble constant of the Hubble-Lemaître law is used to solve the Friedmann equations and find values for $\Lambda(t)$ and $k(t)$. 


\section{Equation of state for the temperature, pressure, volume}

The photon gas equation that applies when photon numbers are high enough to be considered a gas $(\mathrm{N}>>1)$ is written as:

$$
\mathrm{PV}=\frac{\zeta(4)}{\zeta(3)} \mathrm{k}_{\mathrm{b}} \mathrm{N} \mathrm{T}=\mathrm{f}(\mathrm{t})
$$

where $f(t)$ represents a function of cosmic time. Observations show that the universe is expanding with time $\mathrm{r}(\mathrm{t})$. Expansion of the universe is isotropic ( $\dot{r}$ isotropic) and in accordance with the Hubble-Lemaître law. The volume V of space (photon propagation) thus generated is isotropic (large-scale isotropic, $\dot{V}$ ). The mechanism behind the evolution pattern for $\mathrm{V}$ is unknown but, as we will see later, it is represented by the evolution of energy associated with curvature k. It starts with the initial Planck time $t_{p}$, and time evolves freely as $t+t_{p}$. At every step, $t_{p}, V, T$ and P evolve, but the triggering mechanism for this evolution is unknown. $\mathrm{V}, \mathrm{T}$ and $\mathrm{P}$ evolve in some sort of sequence, which is probably as follows: $t+t_{p}, V+d V, N+d N, T-d T, P-d P, E-d E$. The expanding volume (spacetime) is a sphere whose radius evolves in line with cosmic time. The HubbleLemaître law takes the following simple form:

$$
\dot{r}=\mathrm{Hr}=1 / \mathrm{t}
$$

In this version, $\mathrm{H}$ varies according to cosmic time. We can observe $\mathrm{H}$ at $\mathrm{t}_{0}$, written as $\mathrm{H}_{0}$ ( 67,8[km s $\left.\left.{ }^{-1} M p c^{-1}\right]\right)$ (Planck Collaboration, Aghanim, Akrami et al., 2018). This yields $\mathrm{r}=\mathrm{ct}$ $+r_{\alpha}$ as the mean evolution of $r$ over time. The radius can undergo local, spontaneous variations that are different than ct, but the average is still equal to ct.

Let us write the equation of state for photon gas in the form of the variation, freely choosing the negative form of the variations, which allows to denote the possible existence of a singularity at the beginning of the evolution of the universe. Moreover, CMB observations reveal a decay of T: 


$$
\frac{P V}{T}=\frac{(P-d P)(V-d V)}{T-d T}=\mathrm{f}(\mathrm{t})
$$

Developing the right-hand side yields:

$$
\frac{d T}{T}=\frac{d V}{V}+\frac{d P}{P}-\frac{d P d V}{P V}
$$

The final term on the right is retained as it contains the potential existence of a singularity at the beginning of the evolution of the universe.

Let us develop $\mathrm{V}, \mathrm{dV}, \mathrm{P}$ and $\mathrm{dP}$ :

$$
\begin{gathered}
\mathrm{V}=\frac{4 \pi}{3} \mathrm{r}^{3}=\frac{4 \pi}{3}\left(\mathrm{ct}+\mathrm{r}_{\alpha}\right)^{3} \\
\dot{V}=\frac{d V}{d r} \dot{r}=4 \pi \mathrm{r}^{2} \mathrm{H} \mathrm{r}=3 \mathrm{H} \mathrm{V} \\
\mathrm{dV}=3 \mathrm{HV} \mathrm{dt} \\
\frac{d V}{V}=3 \mathrm{H} \mathrm{dt}
\end{gathered}
$$

For a photon gas associated with a blackbody considered in a state of equilibrium $(\mathrm{N}>>1)$, radiation pressure is expressed as:

$$
\begin{gathered}
\mathrm{P}=\frac{4 \sigma}{3 c} \mathrm{~T}^{4} \\
\frac{d P}{d T}=\frac{16 \sigma}{3 c} \mathrm{~T}^{3} \\
\frac{d P}{P}=4 \frac{d T}{T} \\
\frac{d P d V}{P V}=12 \mathrm{H} \mathrm{dt} \frac{d T}{T}
\end{gathered}
$$

Finally, we derive the following specific equation for the evolution of photon gas temperature in a context of expansion of the universe $(\mathrm{N}>>1)$ :

$$
\frac{d T}{T}=\frac{H d t}{-1+4 H \widetilde{d t}}
$$

The equation for temperature variations in line with the Hubble constant yields different scenarios of evolution for $\mathrm{T}(\mathrm{t})$. First, integration creates a problem since $\mathrm{dt}$ appears in both the numerator 
and denominator. The presence of $\widetilde{d t}$ in the denominator is caused by the term $\mathrm{dVdP} / \mathrm{VP}$. If this term is left aside, we get a conventional form of $-\mathrm{H}$ dt. Integration can be done by considering the process as a summation along cosmic time $\mathrm{t}$ for the numerator $\mathrm{dt}$, with $\mathrm{H} /(-1+4 \mathrm{H} \widetilde{d t})$. Then, the term $4 \mathrm{H} \widetilde{d t}$ can be processed in various ways. Moreover, the value of $\mathrm{H}$ can vary according to different expansion scenarios. In this version of the model, we assume that the Hubble constant decreases monotonically with time. Let us assume that this term remains constant for the main integration of $\mathrm{dt}$, therefore:

$$
\mathrm{T}(\mathrm{t})=\frac{a_{4}}{-t+4 \widetilde{d t}}
$$

where $\mathrm{H}=1 / \mathrm{t}$, or $r \ddot{\gamma} r=H^{2}+\dot{H}=0$, or still $\mathrm{q}=0$ (for the boundary of the universe).

Note that the acceleration factor q of the boundary of the universe is zero, but we will see later that it is not zero for the mass of the universe.

The equation for $\mathrm{T}$ in relation to cosmic time yields interesting characteristics. First, two constants, or unknowns, a4 and $\widetilde{d t}$, are required to determine the evolution process of $\mathrm{T}$. Second, $\widetilde{d t}$ is normally positive, because time is positive and so is $\widetilde{d t}$. Third, $\widetilde{d t}$ can be considered a time limit in the flow of time $\mathrm{t}$, which is causal. The smallest $\widetilde{d t}$ time limit could be a unit of Planck time, $\mathrm{t}_{\mathrm{p}}$.

\section{State equation, evolution of photon gas, temperature, volume and pressure}

No data is available on the evolution of temperature in the universe due to the limited time since the beginning of $\mathrm{T}$ measurements. $\mathrm{CMB}$ temperature has been measured, as well as spatial variation $\Delta T$. We also know Planck temperature, $T_{p}$, which is normally considered the maximum temperature of any element. If we take $T(0)=T_{p}$, (Lima and Trodden, 1996), which denotes the maximum energy in the universe at positive temperature, we get:

$$
\mathrm{T}(0)=\mathrm{T}_{\alpha}=\mathrm{T}_{\mathrm{p}}=\frac{a_{4}}{-4 \widetilde{d t}}
$$


And then

$$
\mathrm{a}_{4}=-4 \widetilde{d t} \mathrm{~T}_{\mathrm{p}}
$$

If we assume that the temperature must remain positive at the beginning and all along the cosmic timeline, then the constant $\mathrm{a}_{4}$ is also positive. This choice of positive temperature is debatable, and a negative temperature at the beginning of the universe leads to a positive temperature after a time delay of $4 \widetilde{d t}$. However, the use of a negative temperature requires the support of an extra element, which is not included in this model.

Let us define the age of the universe as $\mathrm{t}_{\Omega}$, and $\mathrm{CMB}$ temperature as $\mathrm{T}_{\Omega}$, or that of the universe as we see it today. Therefore:

$$
\mathrm{T}\left(\mathrm{t}_{\Omega}\right)=\mathrm{T}_{\Omega}=\frac{-4 d t T_{p}}{-t_{\Omega}+4 \widetilde{d t}}
$$

The value of $\widetilde{d t}$ for this condition is:

$$
\widetilde{d t}=\frac{T_{p} t_{\Omega}}{4\left(T_{\Omega}+T_{p}\right)}=\mathrm{b} / 4
$$

To develop an equation for $\mathrm{T}$, we can start with:

$$
\mathrm{T}(\mathrm{t})=\frac{C_{1}}{-t+b}=\frac{\frac{T_{\Omega} T_{p} t_{\Omega}}{\left(T_{\Omega}-T_{p}\right)}}{-t+\frac{T_{\Omega} t_{\Omega}}{\left(T_{\Omega}-T_{p}\right)}}
$$

Finally, we can assume $\left(\mathrm{T}_{\Omega}-\mathrm{T}_{\mathrm{p}}\right) \sim-\mathrm{T}_{\mathrm{p}}$, then the final expression for $\mathrm{T}$ is:

$$
\mathrm{T}(\mathrm{t})=\frac{-t_{\Omega} T_{\Omega}}{-t-\frac{t_{\Omega} T_{\Omega}}{T_{p}}}
$$

The equation for $\mathrm{T}$ includes a potential singularity for negative $\mathrm{T}_{\mathrm{p}}$, as:

$$
\mathrm{t}_{\mathrm{s}}=\frac{t_{\Omega} T_{\Omega}}{T_{p}} \sim \frac{2.7 \mathrm{~K}}{1.4 \times 10^{32} \mathrm{~K}} \mathrm{t}_{\Omega} \sim 1.93 \times 10^{-32} \mathrm{t} \Omega
$$

For example, for $\mathrm{t}_{\Omega \min }=4.351 \times 10^{17}(13.8[\mathrm{~Gy}])$, a singularity is obtained around:

$$
\mathrm{t}_{\mathrm{s}}=8.4 \times 10^{-15}[\mathrm{~s}]
$$


The result is far too removed from the normally accepted value where the inflation of space occurs $\left(\sim 10^{15}\right.$ removed from the value $\sim 10^{-30}[s]$ (Guth and Steinhardt, 1984)).

The most important point to note about this timespan or delay, expressed as $b=-t_{\Omega} T_{\Omega} T_{P}^{-1}$, is the fact that it allows to slow the decrease in temperature down to a characteristic value of $\sim 10^{-14}$ [s]. We will see that during that delay, the number of photons increases at a quasi-constant temperature and pressure, which allows finding a possible explanation for the event horizon problem.

Photon gas pressure is expressed as $(\mathrm{N}>>1)$ :

$$
\mathrm{P}=\frac{4 \sigma}{3 c} \mathrm{~T}^{4}=\frac{4 \sigma}{3 c}\left[\frac{C_{1}}{-t+b}\right]^{4}=\frac{4 \sigma}{3 c} T_{\Omega}^{4}\left[\frac{t_{\Omega}}{-t-\frac{t_{\Omega} T_{\Omega}}{T_{p}}}\right]^{4}
$$

Volume is expressed as:

$$
\mathrm{V}=\frac{\frac{\zeta(4)}{\zeta(3)} k_{b} \mathrm{~N} \mathrm{~T}}{P}=\frac{4 \pi}{3}\left(\mathrm{ct}+\mathrm{r}_{\alpha}\right)^{3}
$$

At the beginning, the volume is:

$$
\mathrm{V}(0)=\frac{4 \pi}{3}\left(\mathrm{ct}+r_{\alpha}\right)^{3}=\frac{4}{3} \pi l_{p}^{3}
$$

For the number of photons in line with temperature:

$$
\mathrm{N}=\mathrm{V} \mathrm{n}=\mathrm{V} \frac{2 \zeta(3)}{\pi^{2}}\left(\frac{2 \pi k_{b} T}{h c}\right)^{3}=\frac{4 \pi}{3}\left(\mathrm{ct}+\mathrm{r}_{\alpha}\right)^{3} \frac{2 \zeta(3)}{\pi^{2}}\left(\frac{2 \pi k_{b} T}{h c}\right)^{3}
$$

With $\mathrm{r}_{\alpha}=l_{p}$ (Planck length).

\section{Increase in the number of photons}

If the expressions $l_{p}$ and $T_{p}$ at $t=0$ are used, the number of photons at the beginning of the universe, $(\mathrm{t}=0)$, is:

$$
\mathrm{N}(0)=\frac{4 \pi}{3} l_{p}^{3} \frac{2 \zeta(3)}{\pi^{2}}\left(\frac{2 \pi k_{b} T_{p}}{h c}\right)^{3}=\frac{4 \pi}{3} \frac{2 \zeta(3)}{\pi^{2}}\left(\frac{h G}{2 \pi c^{3}}\right)^{3 / 2}\left(\frac{2 \pi k_{b}\left(\frac{h c^{5}}{2 \pi G k_{b}^{2}}\right)^{1 / 2}}{h c}\right)^{3}=\frac{64 \zeta(3)}{24 \pi}=\frac{8 \zeta(3)}{3 \pi}=1,02 !
$$


The result is not exactly equal to one, and the reason for this is unknown. Of course, the reason behind the existence of the first photon is also unknown! We see that at the beginning, only one photon is present in the original Planck volume. The expression of the number of photons making up the most part of the energy relative to the age of the universe is, $t_{\Omega}$. Expression of the number of photons in relation to cosmic time is:

$$
\mathrm{N}(\mathrm{t})==\frac{8 \zeta(3)}{3 \pi}\left(\frac{2 \pi k_{b} T_{\Omega}}{h c}\right)^{3}\left[\frac{\left(-c T_{p} t_{\Omega}\right) t-\left(l_{p} T_{p} t_{\Omega}\right)}{\left(-T_{p}\right) t-\left(t_{\Omega} T_{\Omega}\right)}\right]^{3}
$$

The cosmic time expression can be used as a progression of $n$ Planck time units, which then yields the following expression of the number of photons in relation to the number of Planck time units:

$$
\mathrm{N}\left(\mathrm{nt}_{\mathrm{p}}\right)=\frac{8 \zeta(3)}{3 \pi}\left(\frac{2 \pi k_{b} T_{\Omega}}{h c}\right)^{3}\left[\frac{\left(-c T_{p} t_{\Omega}\right) n t_{p}-\left(l_{p} T_{p} t_{\Omega}\right)}{\left(-T_{p}\right) n t_{p}-\left(t_{\Omega} T_{\Omega}\right)}\right]^{3}
$$

The above expression of the number of photons relative to time is unusual. Indeed, we find that the number of photons increases according to a geometrical progression of $\sim \mathrm{n}^{3}$ over a characteristic time of $\sim 10^{-9}[\mathrm{~s}]$ for an age of $76.1[\mathrm{~Gy}]$, up to a maximum where it remains constant. However, the energy necessary to expand the number of photons is not known or at least it is not in the electromagnetic form (photonic). This energy of expanding the number of photons could be identified as the one often mentioned void energy. We will see how this progression in the number of photons relative to time will make it possible to solve the complex horizon problem.

The expression trends towards a constant number of photons, $\sim 10^{-9}[\mathrm{~s}](\mathrm{dN} / \mathrm{dt}=0)$. For $t_{\Omega}=76,1$ [Gy] $\left(2,39 \times 10^{18}[\mathrm{~s}]\right)$, we get a constant number of photons:

$$
\mathrm{N}(\infty)=\frac{64 \zeta(3) \pi^{2}}{3}\left(\frac{k_{b} T_{\Omega} t_{\Omega}}{h}\right)^{3} \sim 6,42 \times 10^{89}(\text { constant })
$$

The time period when the number of photons increases geometrically is called the photon epoch (Figs. $1 \& 2$ ). The process leading to photon inflation is unknown but at every time increment, the number of photons increases. However, the increase in energy is caused by photon inflation 
because photon energy remains slightly below Planck energy, $\mathrm{E}_{\mathrm{p}},\left(1.76 \times 10^{9}[\mathrm{~J}]\right)$ until time $\sim 10^{-9}$ $[s]$ (Fig 3).

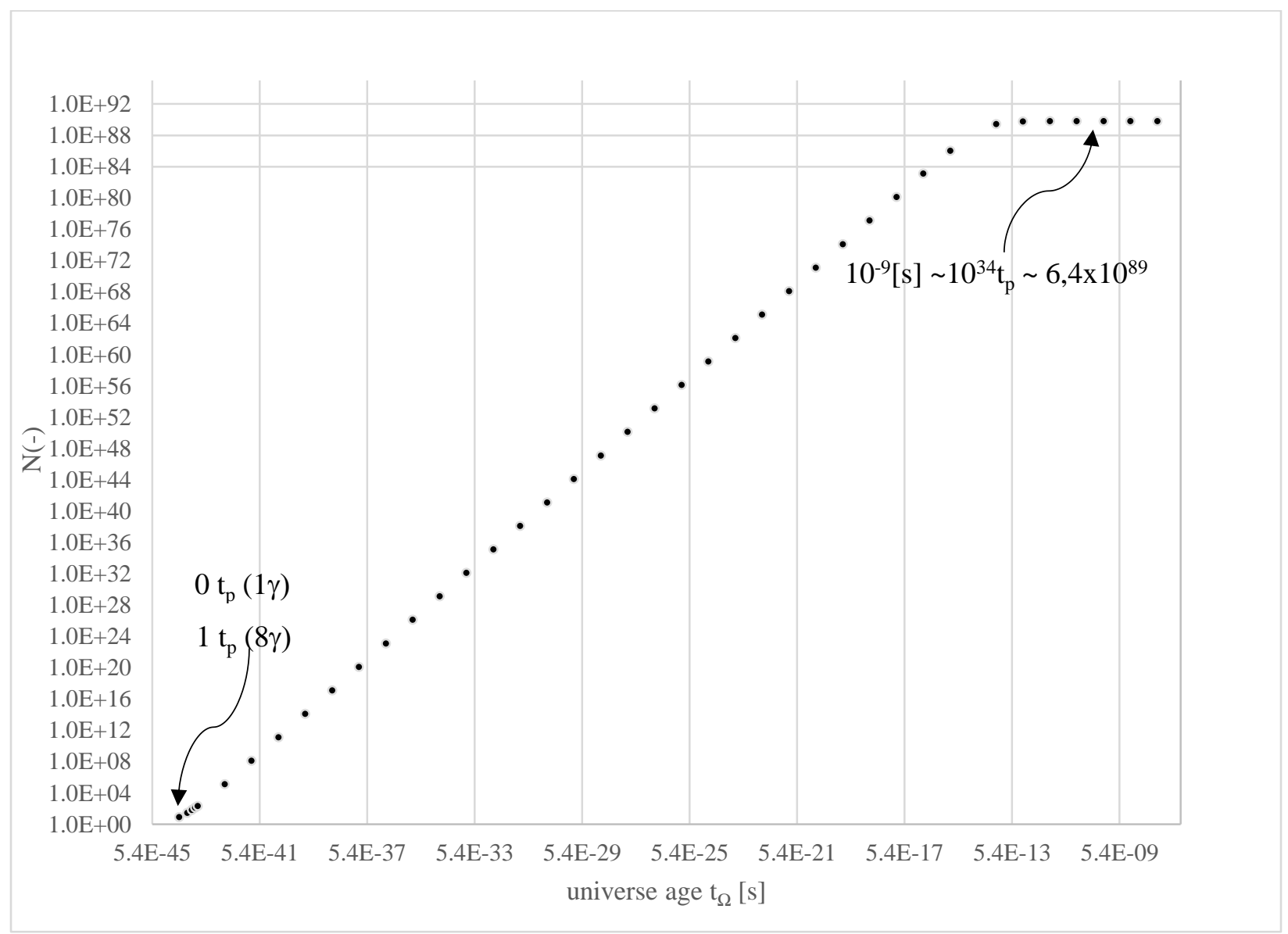

Figure 1: Inflation of photons number from $1 \mathrm{t}_{\mathrm{p}}$ to $1 \times 10^{-6}[\mathrm{~s}]$ 


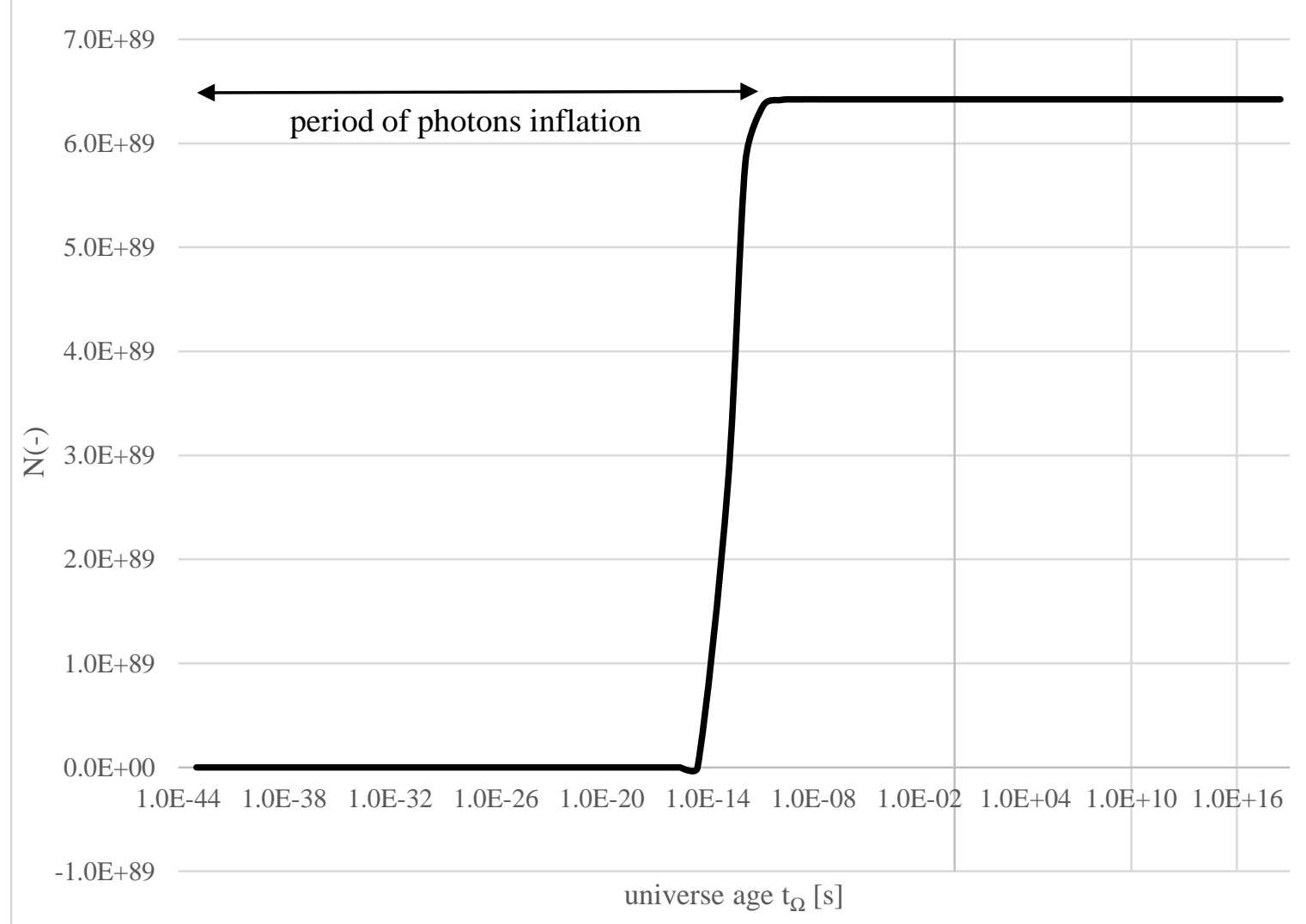

Figure 2: Number of Photons from $1 t_{p}$ to 76,1 [Gy]

\section{Energy gain}

Energy at the beginning of the universe is expressed as the energy of a single photon, the value of which is slightly lower than Planck energy, $\mathrm{E}_{\mathrm{p}}$. For $\mathrm{N}=1$ :

$$
U(0)=0,9 \mathrm{Nk}_{\mathrm{b}} \mathrm{T}_{\mathrm{p}}=0,9 \mathrm{k}_{\mathrm{b}} \mathrm{T}_{\mathrm{p}}=0,9 \mathrm{E}_{\mathrm{p}}=0,9 \mathrm{c}^{2} \sqrt{\frac{c h}{2 \pi G}}=1,76 \times 10^{9}[\mathrm{~J}]
$$

From a macroscopic standpoint, we assume that the universe does not undergo energy transfers with other universes. Also, conventional energy is preserved in relation to time.

Photon gas energy in relation to time can be expressed in several equivalent ways for $\mathrm{N} \gg 1$ :

$$
\mathrm{U}(\mathrm{t})=3 \mathrm{PV}=\frac{4 \sigma}{c} \mathrm{~T}^{4} \mathrm{~V}=3 \frac{\zeta(4)}{\zeta(3)} \mathrm{N} \mathrm{kb}_{\mathrm{b}} \mathrm{T} \sim 2,7 \mathrm{~N} \mathrm{~kb}_{\mathrm{b}} \mathrm{T}
$$


With the expression for $\mathrm{N}(\mathrm{t})$ obtained earlier:

$$
\mathrm{U}(\mathrm{t})=2,7 \mathrm{~N} \mathrm{~kb} \mathrm{~T}=64 \pi^{2} \zeta(4)\left(\frac{c t+r_{\alpha}}{h c}\right)^{3}\left(k_{b} T\right)^{4}
$$

It can be written as:

$$
\mathrm{U}(\mathrm{t})=\left(\frac{64 \zeta(4) \pi^{2} k_{b}^{4}}{h^{3} c^{3}}\right)\left(\frac{\left(c t+l_{p}\right)^{3}}{(-t+b)^{4}}\right)\left(t_{\Omega} T_{\Omega}\right)^{4}=U_{0}\left(\frac{\left(c t+l_{p}\right)^{3}}{(-t+b)^{4}}\right)\left(t_{\Omega} T_{\Omega}\right)^{4}
$$

Or still as:

$$
\mathrm{U}(\mathrm{n})=\left(\frac{64 \zeta(4) \pi^{2}}{h^{3} t_{p}}\right)\left(\frac{(n+1)^{3}}{\left(n+k^{\prime}\right)^{4}}\right)\left(k_{b} t_{\Omega} T_{\Omega}\right)^{4}
$$

Where $\mathrm{n}$ is the whole number of Planck time units, $\mathrm{t}_{\mathrm{p}}$, and $\mathrm{k}$ ' is a constant of universe (CMB temperature is considered constant, as well as the age of the universe, $76,1[\mathrm{~Gy}]$ ), therefore:

$$
\begin{gathered}
\mathrm{n}=\mathrm{t} / \mathrm{t}_{\mathrm{p}} \\
k^{\prime}=\left(\frac{t_{\Omega}}{t_{p}}\right)\left(\frac{T_{\Omega}}{T_{p}}\right)=3,57 \times 10^{11} t_{\Omega}=8,57 \times 10^{29}[s]=2,7 \times 10^{13}[G y]
\end{gathered}
$$

For $\mathrm{n}=0,(\mathrm{~N}=1)$, we get:

$$
\mathrm{U}(0)=0,9 \mathrm{k}_{\mathrm{b}} \mathrm{T}_{\mathrm{p}}=\left(\frac{64 \zeta(4) \pi^{2}}{3 h^{3} t_{p}}\right)\left(\frac{(1)^{3}}{\left(k^{\prime}\right)^{4}}\right)\left(k_{b} t_{\Omega} T_{\Omega}\right)^{4}=\left(\frac{64 \zeta(4) \pi^{2} k_{b}^{4} t_{p}^{4}}{3 h^{3}}\right) T_{p}^{4}=0,9 \mathrm{E}_{\mathrm{p}}
$$

For $\mathrm{t}=t_{\Omega},(\mathrm{N}>>1)$ we get:

$$
\mathrm{U}\left(\mathrm{t}_{\Omega}\right)=\left(\frac{64 \zeta(4) \pi^{2} k_{b}^{4} t_{p}^{4} k^{\prime}}{h^{3}}\right) \frac{T_{p}^{4}}{t_{\Omega}}=\left(\frac{64 \zeta(4) \pi^{2} k_{b}^{4} T_{\Omega}^{4}}{h^{3}}\right) t_{\Omega}^{3}
$$

Maximum energy is reached for $\dot{U}\left(t_{\max }\right)=0$, or:

$$
\frac{3 T}{h}+4 \dot{T}\left(\frac{t}{h}+\frac{l_{p}}{h c}\right)=0
$$

We get:

$$
t_{\max }=3 t_{s}-4 t_{p}=3 \frac{t_{\Omega} T_{\Omega}}{T_{p}}-4 t_{p}=1,93 \times 10^{-32} t_{\Omega}-4 t_{p}
$$

For $\mathrm{t}_{\Omega}=76.1[\mathrm{~Gy}]$, we get:

$$
t_{\max }=1,38 \times 10^{-13}[s]=2,57 \times 10^{30} \mathrm{t}_{\mathrm{p}}
$$


And for $\left(\mathrm{t}_{\Omega}=76,1[\mathrm{~Gy}]\right)$ :

$$
U_{\max }\left(t_{\max }\right)=3,57 \times 10^{98}[\mathrm{~J}]
$$

Mass has not yet been created at this time because the temperature is in the order of $3,5 \times 10^{31}[\mathrm{~K}]$. To get an idea of the sheer magnitude of energy, assuming that the entire mass created is in the order of $10^{52}[\mathrm{~kg}]$, with relativistic energy-mass equivalence $(\beta=0,9)$, this corresponds to $2 \times 10^{69}$ $[\mathrm{J}]$; still an infinitissimal fraction of the energy in the universe.

Figure 4 below shows a graph for $\mathrm{U}(\mathrm{t})$ at $\mathrm{t}_{\Omega}=76,1[\mathrm{~Gy}]\left(2,39 \times 10^{18}[\mathrm{~s}]\right)$.

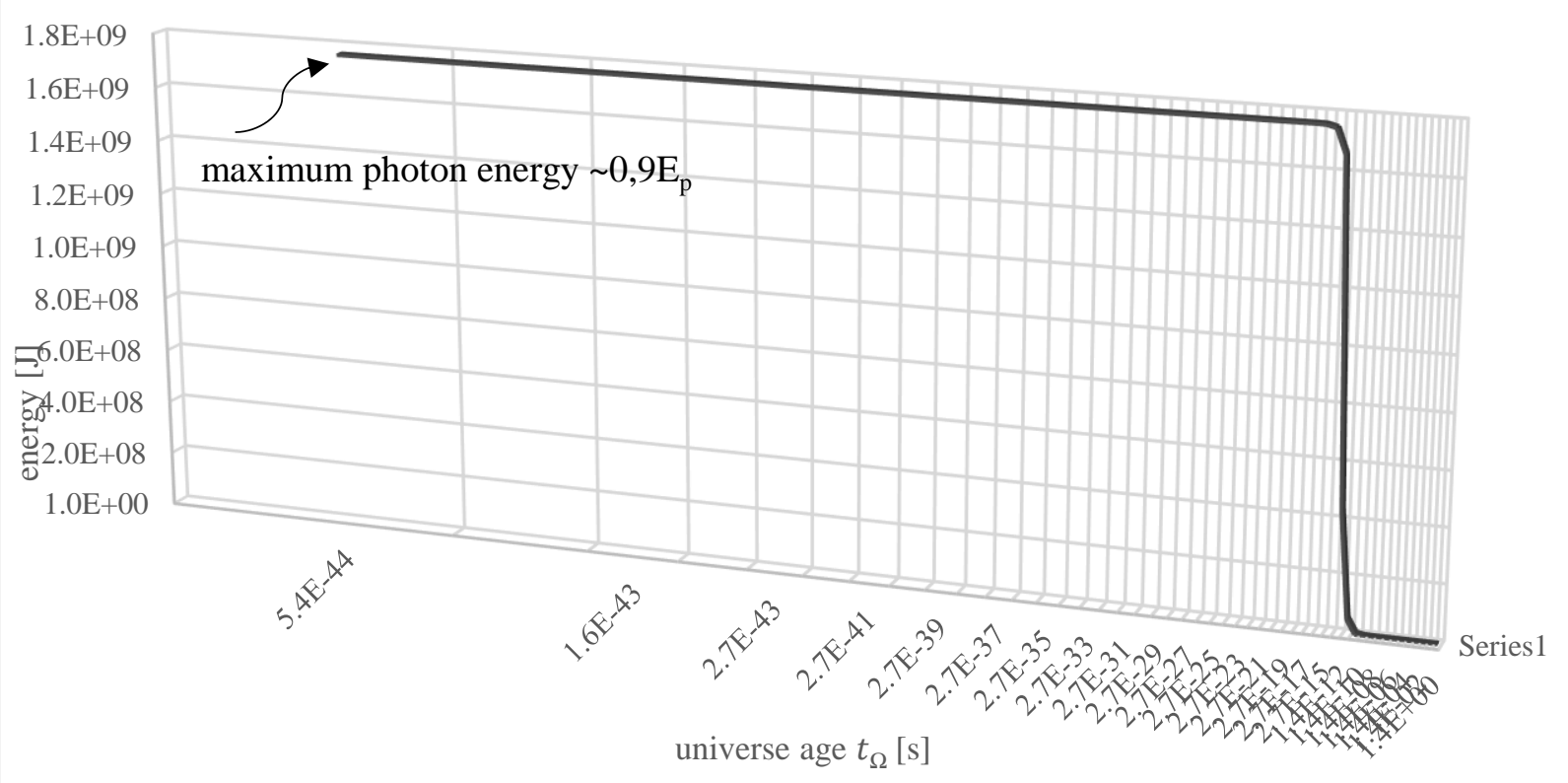

Figure 3: Photon mean energy from $1 \mathrm{t}_{\mathrm{p}}$ to $1,4[\mathrm{~s}]$ 


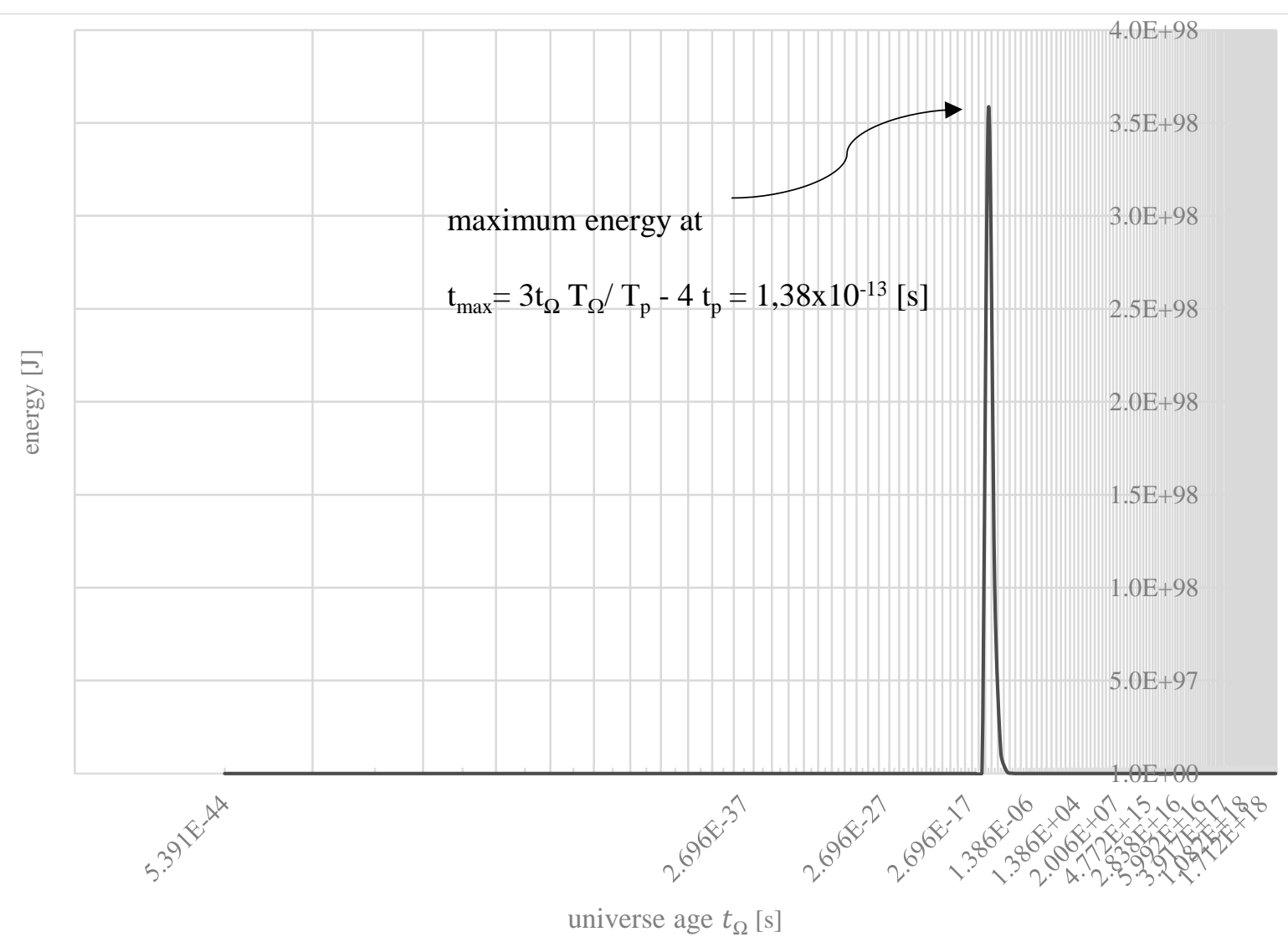

Figure 4: Universe total energy from $1 \mathrm{t}_{\mathrm{p}}$ to $76,1[\mathrm{~Gy}]$

The energy gain, by a factor of $10^{89}$, can be explained by the increase in the number of photons, also by a factor of $10^{89}$, during time period named photon inflation period, or $1.38 \times 10^{-13}$ [s]. During that photon inflation period, the number of photons increases, but the energy of each photon remains approximately the same as the Planck energy, $\mathrm{E}_{\mathrm{p}}$ (Fig 3). Moreover, during that timespan, the temperature, as well as the pressure, remain practically stable at $\mathrm{T}_{\mathrm{p}}$ and $\sim 0.2 \mathrm{P}_{\mathrm{p}}$ (Figs. $5 \& 6$ ). Over that time, volume increases by a factor of $10^{86}$. Photons are created and this remains unexplained, but this is due to the expansion of the universe volume, $\mathrm{V}$ and the availability of an unknown energy. Such colossal energy comes from an existing potential which enchances the 
proliferation of photons, since nothing other than the above equations can predict energy levels. The number of photons increases in a geometrical progression of nearly $\mathrm{n}^{3}$, where $\mathrm{n}$ is the number of Planck time units, $t_{p}$.

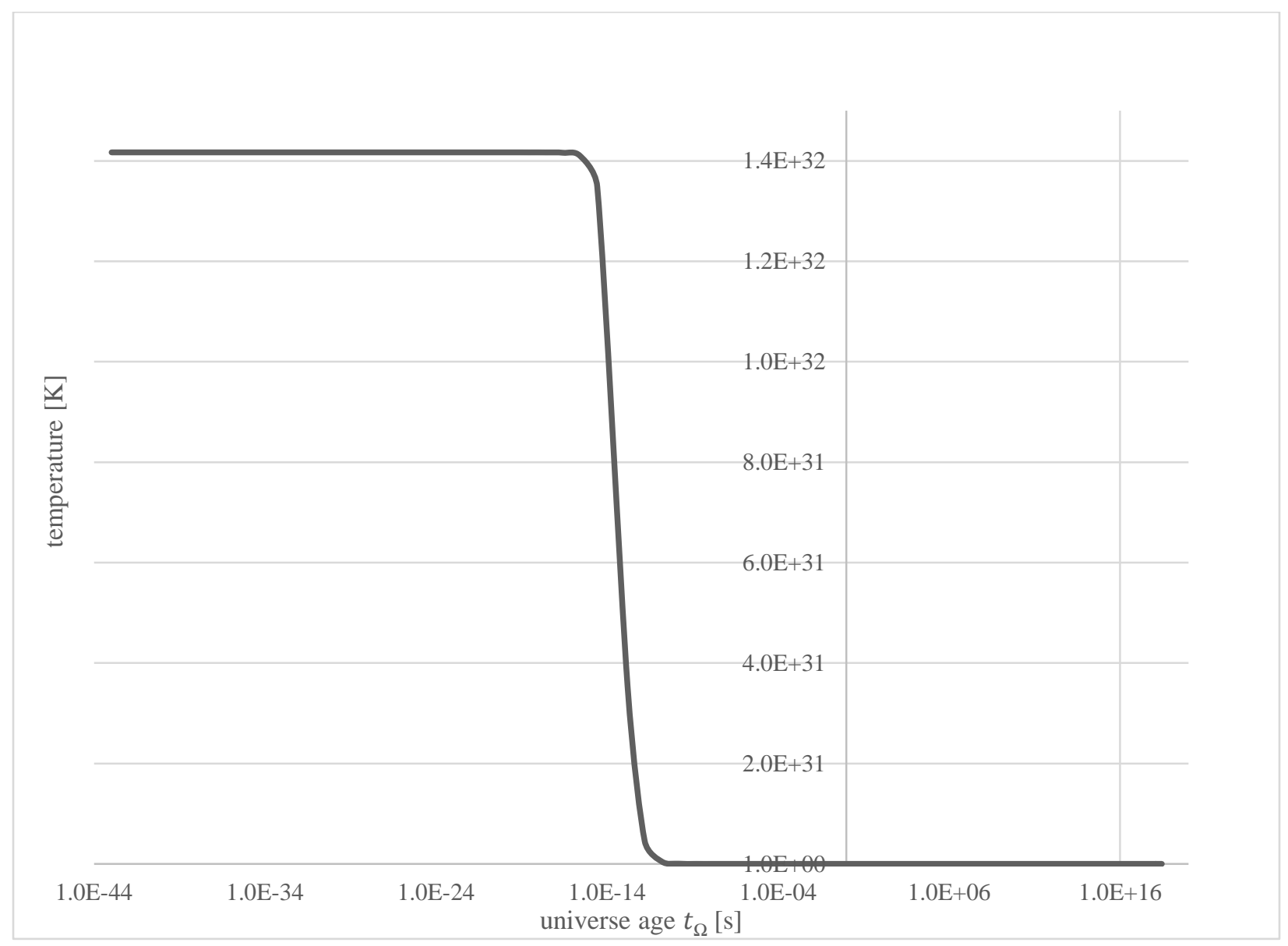

Figure 5: Temperature from $1 \mathrm{t}_{\mathrm{p}}$ to $76,1[G y]$ 


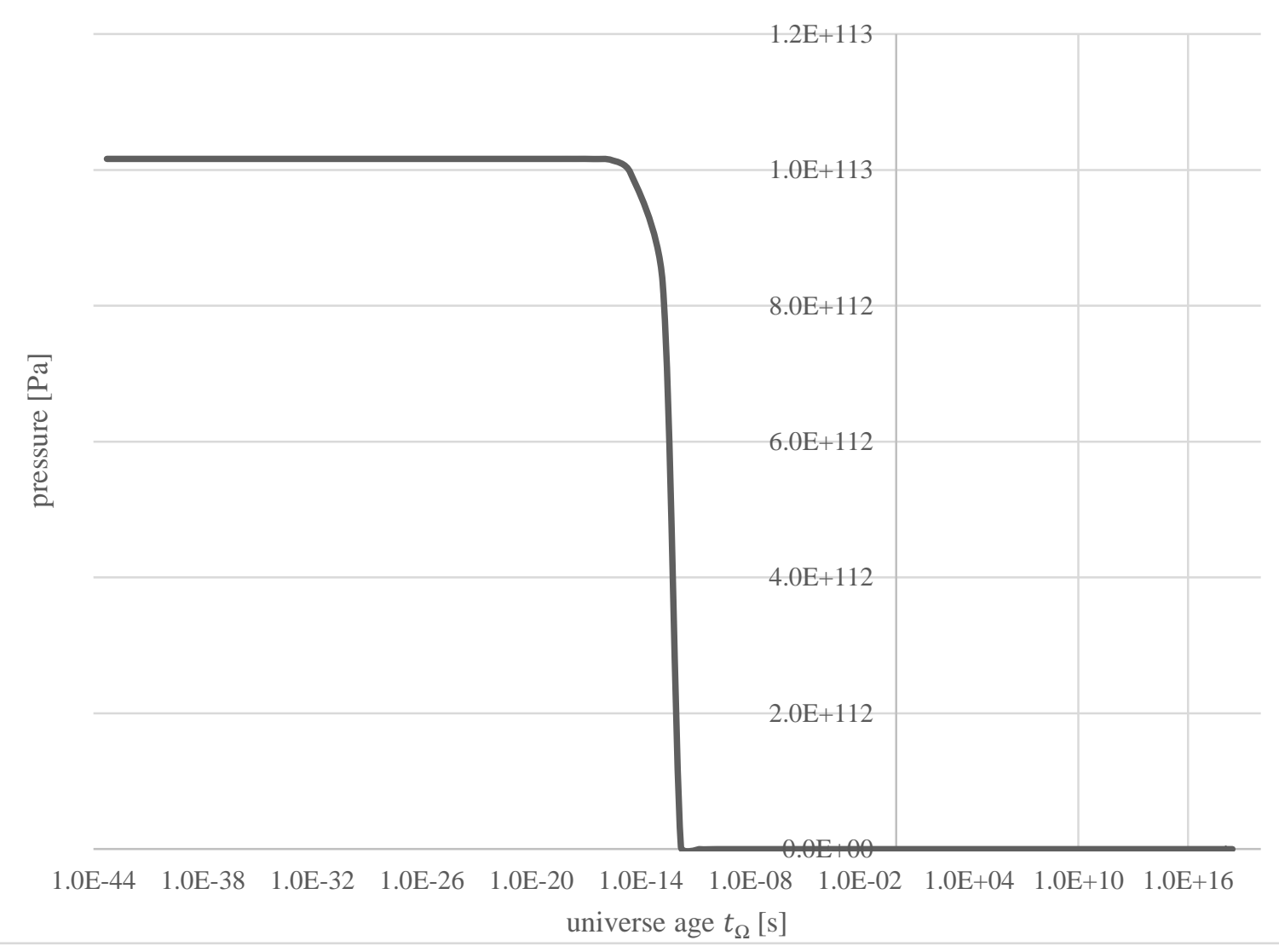

Figure 6: Pressure from $1 \mathrm{t}_{\mathrm{p}}$ to $76,1[G y]$

\section{A possible solution to the horizon problem?}

We have seen that the number of photons increases in geometric progression of $\sim n^{3}$, where $n$ is the number of Planck time units, $\mathrm{t}_{\mathrm{p}}$. Let us find an expression for the volume of the universe in relation to the number of Planck time units, $n$, the boundary is moving at the speed of light:

$$
V\left(n t_{p}\right)=\frac{4 \pi}{3}\left(c(n+1) t_{p}\right)^{3}
$$

During the photon inflation period, the volume occupied by photons in relation to their number, $\mathrm{N}$, the Wien's law, and the number of Planck time units can be estimated as:

$$
V_{\gamma}\left(n t_{p}\right) \sim N \frac{4 \pi}{3}(\lambda)^{3}=N \frac{4 \pi}{3}\left(\frac{\sigma_{w}}{T}\right)^{3}
$$

With the equation found for temperature $\mathrm{T}$ : 


$$
V_{\gamma}\left(n t_{p}\right)=N \frac{4 \pi}{3}\left(\frac{\sigma_{w}}{T}\right)^{3}=N \frac{4 \pi}{3}\left(\frac{\sigma_{w}}{\frac{-t_{\Omega} T_{\Omega}}{-n t_{p}-\frac{t_{\Omega} T_{\Omega}}{T p}}}\right)^{3}
$$

Let us express the photon volume quotient to the volume of the universe relative to the number of Planck time units, $\mathrm{n}$, and the number of photons $\mathrm{N}$.

$$
\frac{V_{\gamma}\left(n t_{p}\right)}{V\left(n t_{p}\right)}=\frac{N\left(\frac{\sigma_{w}}{\frac{-t_{\Omega} T_{\Omega}}{-n t_{p}-\frac{t_{\Omega} T_{\Omega}}{T_{p}}}}\right)^{3}}{c^{3}(n+1)^{3} t_{p}^{3}}
$$

After manipulation, the expression can be written as:

$$
\frac{V_{\gamma}\left(n t_{p}\right)}{V\left(n t_{p}\right)}=\left(\frac{N}{(n+1)^{3}}\right)\left(\frac{\sigma_{w}}{c t_{p} t_{\Omega} T_{\Omega} T_{p}}\right)^{3}\left(n t_{p} T_{p}-t_{\Omega} T_{\Omega}\right)^{3}
$$

In the above expression, the only variables that evolve are the number of Planck time units, n, and number of photons, $\mathrm{N}$. The value of the quotient found for the entire age of the universe is:

$$
\frac{V_{\gamma}\left(n t_{p}\right)}{V\left(n t_{p}\right)} \sim 2,06 \text { (constant) }
$$

What does this result mean? We have found that the volume occupied by photons, which increases in geometric progression, is always slightly higher than the volume of the universe, and its boundary is moving at the speed of light. Obviously, the value 2 is not accurate because the photons are contained within the volume of the universe. The important value here is the constant. Now, we can imagine the process occurring at every unit of Planck time. The number of photons potentially increases around the volume created (at the boundary?) at every unit of Planck time; the new photons exchange through high-energy photon-photon interactions. 
Moreover, at every unit of Planck time, the already existing photons also undergo $\gamma \gamma$ exchange. This $\gamma \gamma$ exchange process is made possible by the quotient between the number of new photons around the boundary of the existing photons at Planck time, prior to the progression from the maximum 8 to the minimum 1, or 8 at the first unit of Planck time, down to 1 when the number of photons no longer increases, or:

$$
\frac{N\left((n+1) t_{p}\right)}{N\left(n t_{p}\right)} \sim \frac{8}{1}(n=0) \rightarrow \frac{10^{89}}{10^{89}}\left(n>10^{34}\left(10^{-9}[\mathrm{~s}]\right)\right)=1
$$

This is a very important result, the $\gamma \gamma$ exchange are made possible when the number of photons increases further (ratio $\rightarrow 1$ ). This occurs around $10^{-9}[s]$ after the beginning. Therefore, after that time, the $\gamma \gamma$ exchange remain causal. Moreover, during the photon inflation period, when the $\gamma \gamma$ exchange are not entirely causal, we note that the temperature is steady at Planck temperature (Fig. 6). Hence, even during the photon inflation period, the information exchange between photons cannot be entirely causal, that information is not necessary from a thermodynamic standpoint because the states of $\mathrm{T}$ and $\mathrm{P}$ remain more or less constant (Fig. 7). 


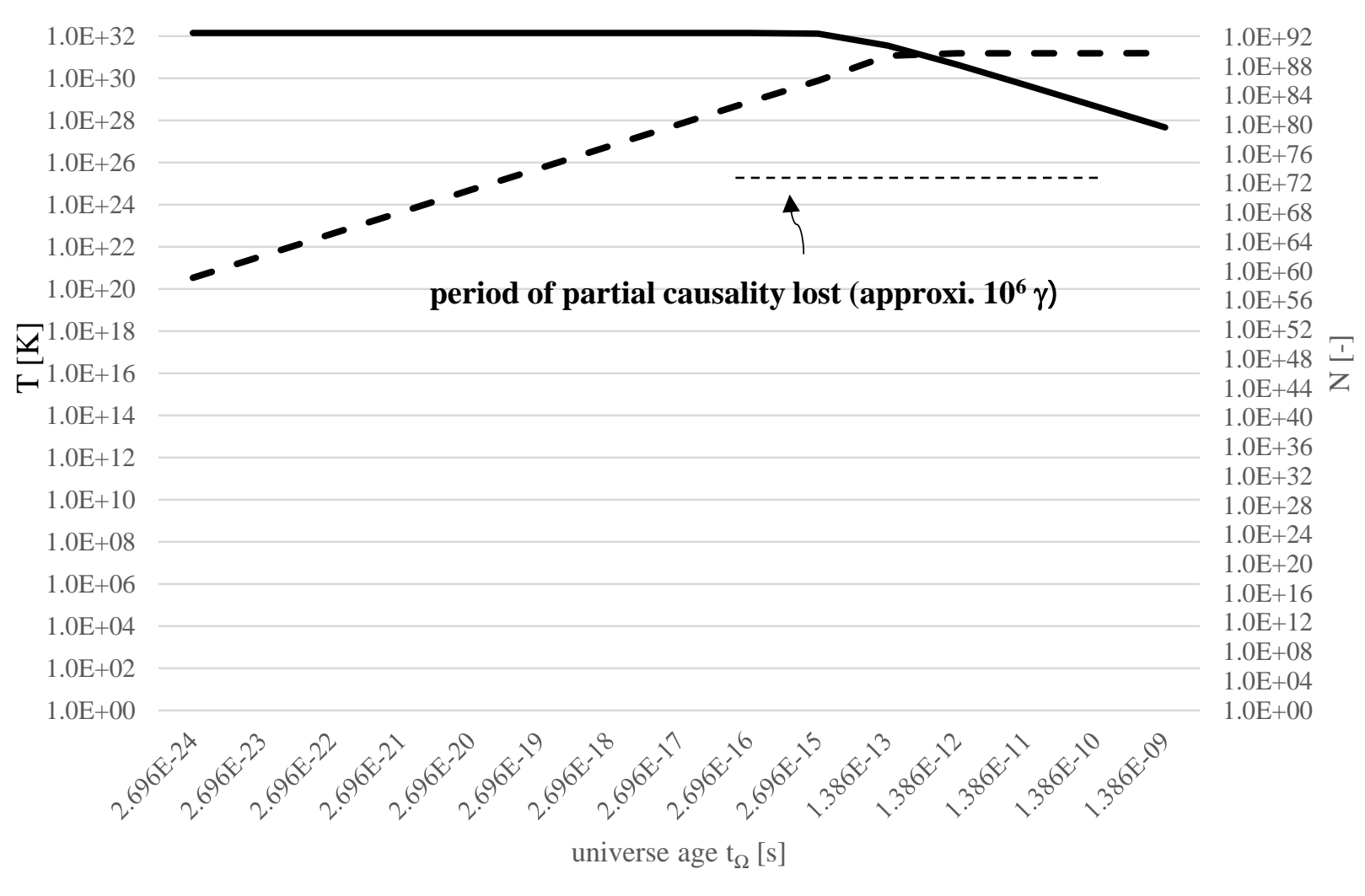

Figure 7: Number of photons and temperature function of cosmic time from $10^{-24}[\mathrm{~s}]$ to $10^{-9}[\mathrm{~s}]$

This mechanism makes it possible to solve the horizon problem for the photon inflation period, or energy creation period, if the high-energy $\gamma \gamma$ exchange principle is accepted. Photon-photon exchange are a fact that has been confirmed at CERN (Kłusek-Gawenda, Lebiedowicz and Szczurek, 2016). Photon exchange energy, $\gamma \gamma$, for that experiment was an estimated $\sim 15-20$ [GeV], while the energy of photons at the beginning was $\sim 0.9 \mathrm{Ep}$, or $\sim 10^{19}[\mathrm{GeV}]$. Of course, this goes beyond the purpose of this paper since $\gamma \gamma$ exchange will require much more study. However, the process makes it possible to solve the event horizon problem, as the photon energy volume is always in phase with that of the volume of the universe. In brief, these periods are:

$$
0<t<\sim 10^{-16}[\mathrm{~s}]\left(\mathrm{z} \sim 10^{33}\right) \text { (causality, T constant) }
$$




$$
\begin{gathered}
\sim 10^{-16}[\mathrm{~s}]<t<\sim 10^{-9}[\mathrm{~s}]\left(\mathrm{z} \sim 10^{26}\right) \text { (partial causality) } \\
\sim 10^{-9}[\mathrm{~s}]\left(\mathrm{z} \sim 10^{26}\right)<t \text { (causality, N constant) }
\end{gathered}
$$

The CMB is at $\mathrm{z} \sim 1100$, or well after the start of the causality recovery period. We will see that the last scattering surface of the model is $\sim 69[\mathrm{My}]$ after the beginning. This leaves $\sim 10^{58}$ Planck time units to restore causality. It can be reasonably assumed that at recombination time the universe had enough time to recover all of the causality, and that is why we can observe isotropy in the CMB (McCoy, 2014).

\section{Early baryogenesis (protons, neutrons) and leptons (electrons, neutrinos)}

Interactions between photons and matter are complex and beyond the scope of this paper. Moreover, relativistic effects have to be considered as particle speeds approach the speed of light upon creation. In this paper, we describe a creation mechanism for the main particles ( $\mathrm{p}, \mathrm{n}$, e and v) to demonstrate the coherence of the model. During early baryogenesis, at very high temperature $\left(\mathrm{mc}^{2}<<\mathrm{kT}\right)$, the Maxwell-Juttner M-J (relativist) statistical law is used to predict particle properties (fermions and letpons). Moreover, the presence of antiparticles must be considered, along with the creation-annihilation process. In this paper, we want to estimate the total barionic mass produced at the end of baryogenesis. We are able to estimate the full potential of mass creation in the universe using the mass-energy equivalence, since we are estimating total energy. The following expression is used to find the mass creation potential. Note that here, we assume that the energy in the universe is conventional:

$$
M_{p o t}=\frac{\sqrt{1-\beta^{2}} U\left(t_{\Omega}\right)}{c^{2}}=\left(\frac{\sqrt{1-\beta^{2}} 64 \zeta(4) \pi^{2} k_{b}^{4} T_{\Omega}^{4}}{c^{2} h^{3}}\right) t_{\Omega}^{3}
$$

We can see that the mass creation potential is relative to the cube of the age of the universe. For comparison purposes, for a universe aged 13,8 [Gy] $(\beta=0)$, the maximum total mass that can be 
produced is $4,81 \times 10^{48}[\mathrm{~kg}]$, which is $\sim 10^{4}$ smaller than the approximate estimated mass of the universe $\left(10^{52}\right.$ à 53 [kg] $)$ (Carvalho, 1995). This clearly shows that to maintain this estimated mass, the existence of a source of non-conventional energy, or dark energy, has to be considered. Another possibility is to extend the age of the universe. Evidently, the precise mass of the universe is unknown. Supposing an estimated mass variation factor of $10^{2}$ and conventional energy, we have to assume, based on the above equation, that the universe is much older than 13,8 [Gy] (visible universe $\sim 13,8[\mathrm{~Gy}])$. Typically, for a mass potential in the order of $10^{50}$ to $10^{53}[\mathrm{~kg}]$, the age of the universe must be somewhere between 37,9 [Gy] and 379 [Gy]. To estimate the volumic quantity of protons and neutrons created, the Maxwell-Juttner statistical distribution is used, as follows (Cercignani and Medeiros Kremer, 2002):

$$
n_{p, n}=\frac{4 \pi c m_{p, n}^{2} k_{b} T K_{2}(\mu)}{h^{3}} e^{\frac{-m_{p, n} c^{2}}{k_{b} T \sqrt{1-\beta^{2}}}}
$$

With: $\mu=\frac{m_{p, n} c^{2}}{\sqrt{1-\beta^{2}} k_{b} T}$ and $K_{2}(\mu)$ the modified Bessel function of the second kind. In this distribution, the stop temperature for the definitive creation of protons and neutrons must be specified, as well as the relativistic speed of created fermions. The value of $\beta$ poses a problem, in fact, a lower value allows to create more mass and conversely also. We will see further from the energetic form of the Friedmann equation that an average value of $\beta$ can be estimated at $\beta \sim 0,866$. However, the global energy equation imposes a maximum value for beta to $\beta \sim 0,998$ in order to maintain the positive energy balance at the scale of the universe (for the entire cosmic time):

$$
\Delta U=U_{\gamma}-U_{M}=2,7 N k_{b} T-\frac{M_{t o t} c^{2}}{\sqrt{1-\beta^{2}}}>0
$$

The temperature can be estimated based on the total energy of a proton or neutron at $\beta$ : 


$$
\bar{T}_{p r, n e}=\frac{\frac{m_{p r, n e} c^{2}}{\sqrt{1-\beta^{2}}}}{k_{b}}=\frac{C_{1}}{\left(-t_{p r, n e}+b\right)}
$$

This mean photon energy appears at proton and neutron temperature and time, or $t_{p r, n e}$, after the beginning of expansion:

Therefore:

$$
\mathrm{t}_{\mathrm{pr}, \mathrm{ne}}=\mathrm{b}-\frac{k_{b} c_{1}}{\frac{m_{p, n c^{2}}}{\sqrt{1-\beta^{2}}}}=\frac{t_{\Omega} T_{\Omega}}{T_{p}}+\frac{k_{b} T_{\Omega} t_{\Omega}}{\frac{m_{p, n} c^{2}}{\sqrt{1-\beta^{2}}}}=\left[\frac{T_{\Omega}}{T_{p}}+\frac{k_{b} T_{\Omega}}{\frac{m_{p, n} c^{2}}{\sqrt{1-\beta^{2}}}}\right] t_{\Omega}
$$

For $t_{\Omega}=76,1[\mathrm{~Gy}]=2,39 \times 10^{18}[\mathrm{~s}]$ and $\beta=0,9986$, we find:

$$
\begin{gathered}
\mathrm{t}_{\mathrm{pr}} \sim 31345[s] \sim 0,3627[\mathrm{~d}] \text { after the beginning of expansion } \\
\bar{T}_{p r}=2,08 \times 10^{14}[\mathrm{~K}] \\
\mathrm{t}_{\mathrm{ne}} \sim 31303[\mathrm{~s}] \sim 0,3623[\mathrm{~d}] \text { after the beginning of expansion } \\
\bar{T}_{n e}=2,09 \times 10^{14}[\mathrm{~K}]
\end{gathered}
$$

The creation potential (without annihilation, $p \bar{p}$, or disintegration, $n$ ) for protons and neutrons at this time is:

$$
n_{p}=\frac{V 4 \pi c m_{p}^{2} k_{b} T_{p r} K_{2}(\mu)}{e h^{3}}=\frac{16 \pi^{2} c^{4} t_{p r}^{3} m_{p}^{2} k_{b} T_{p r} K_{2}(\mu)}{3 e h^{3}}=2,1700 \times 10^{86}
$$

and neutrons:

$$
n_{n}=\frac{V 4 \pi c m_{n}^{2} k_{b} T_{n e} K_{2}(\mu)}{e h^{3}}=2,1689 \times 10^{86}
$$

Where $\mu=\frac{m c^{2}}{\sqrt{1-\beta^{2}} k_{b} T}$

The creation of neutrons occurs $43[s]$ prior to proton fixation, allowing to capture $p+n$ before complete disintegration of the neutrons $(881[\mathrm{~s}])$. To estimate the final number of protons and neutrons, the respective creation and annihilation of antiparticles must be considered. To do so, 
we assume that baryonic asymmetry prevails according to a normally accepted proportion of one stable baryon created for every $10^{9} p \bar{p}$ and $n \bar{n}$ annihilations (Dolgov, 1998). Moreover, neutrons are captured and disintegrate in an accepted proportion of one neutron captured for every four neutrons disintegrated (the calculated ratio is 0,188 for $43[s]$ of disintegration time). Then, in a universe aged 76,1 [Gy], we estimate the stable masses to be:

$$
\begin{gathered}
\mathrm{M}_{\mathrm{p}} \sim 10^{-9}\left(n_{p} m_{p}+0,8 n_{n} m_{n}\right) \sim 6,53 \times 10^{50}[\mathrm{~kg}] \\
\mathrm{M}_{\mathrm{n}} \sim 0,2 \times 10^{-9} n_{n} m_{n} \sim 7,25 \times 10^{49}[\mathrm{~kg}]
\end{gathered}
$$

However, with the exponential disintegration of neutrons, $\sim 95 \%$ of them will still be available for capture (formation of deuterium at $\beta$ ) after $43[s]$ before the creation of protons.

Also, an equation can be found for the baryon-photon ratio, $\eta_{B}$. Initially assuming that the baryonphoton ratio can be expressed as the proton and neutron creation potential after annihilation and disintegration, expressed in a number of protons (at $\beta$ ) only, after manipulation, we get the following equation and a maximum value for the ratio:

$$
\eta_{B}=\frac{n_{b}\left(t_{p r}\right)}{n_{\gamma}\left(t_{p r}\right)} \sim \frac{2 n_{p}\left(t_{p r}\right)}{N\left(t_{p r}\right)}=10^{-9} \frac{\frac{2 V 4 \pi c m_{p}^{2} k_{b} T_{p r} K_{2}(\mu)}{e h^{3}}}{64 \frac{\zeta(3) \pi^{2}}{3}\left(\frac{k_{b} T p r t p r}{h}\right)^{3}}=10^{-9} \frac{\left(1-\beta^{2}\right) K_{2}(\mu)}{2 e \zeta(3)}=10^{-9} \frac{\left(1-\beta^{2}\right) K_{2}(\mu)}{6,53}
$$

The above constant ratio solely depends on $\beta$ associated with protons during (relativistic) creation, and the modified Bessel function of the second kind, $K_{2}(\mu)$ (Maxwell-Juttner distribution), as well as the numbers, e, and Riemann constant, $\zeta(3)$. The value $10^{-9}$ is the oft-used matter-antimatter annihilation factor, $p \bar{p}$. The maximum value is for $\beta=0$, or $\mu=1$ and $K_{2}(1)=1,62$. Therefore:

$$
\eta_{B}=10^{-9} \frac{\left(1-\beta^{2}\right) K_{2}(\mu)}{6,53}=10^{-9} \frac{1,62}{6,53}=2,48 \times 10^{-10}
$$


The resulting value of $2,48 \times 10^{-10}$ is lower than the results of the estimates yielded by the $\Lambda \mathrm{CDM}$ model (Kirilova and Panayotova, 2015), based on Planck measurements. Indeed, the estimated quotient is not a direct measurement, but rather an estimate that is partly based on $\Lambda \mathrm{CDM}$ model assumptions and observations, or:

$$
\eta_{B}=\frac{n_{b}}{n_{\gamma}}=6,108 \pm 0,038 \times 10^{-10}
$$

However, a small change in the oft-stated $\sim 10^{-9}$ particle-antiparticle annihilation factor and $\beta$ can proportionally change the result.

\section{Electrons}

The Maxwell-Juttner statistical distribution for electrons:

$$
n_{e l}=\frac{4 \pi c m_{e}^{2} k_{b} T K_{2}(\mu)}{h^{3}} e^{\frac{-m_{e} c^{2}}{k_{b} T \sqrt{1-\beta^{2}}}}
$$

With: $\mu=\frac{m_{e} c^{2}}{\sqrt{1-\beta^{2}} k_{b} T}$

This mean energy of photons occurs at stop temperature and electron time, expressed as $t_{\mathrm{el}}$, after the beginning of expansion $(\beta=0,9986)$ :

$$
\bar{T}_{e l}=\frac{C_{1}}{\left(-t_{e l}+b\right)}=\frac{\frac{m_{e} c^{2}}{\sqrt{1-\beta^{2}}}}{k_{b}}=1,13 \times 10^{11}[\mathrm{~K}]
$$

Therefore:

$$
\mathrm{t}_{\mathrm{el}}=\mathrm{b}-\frac{k_{b} C_{1}}{\frac{m_{e} c^{2}}{\sqrt{1-\beta^{2}}}}=\frac{t_{\Omega} T_{\Omega}}{T_{p}}+\frac{k_{b} T_{\Omega} t_{\Omega}}{\frac{m_{e} c^{2}}{\sqrt{1-\beta^{2}}}}=\left[\frac{T_{\Omega}}{T_{p}}+\frac{k_{b} T_{\Omega}}{\frac{m_{e} c^{2}}{\sqrt{1-\beta^{2}}}}\right] t_{\Omega}
$$

For $t_{\Omega}=76,1[\mathrm{~Gy}]=2,39 \times 10^{18}[\mathrm{~s}]$ and $\beta=0,9986$, we get:

$$
\mathrm{t}_{\mathrm{el}} \sim 5,755 \times 10^{7}[s] \sim 666[d] \text { after the beginning of expansion. }
$$


The electron creation potential (without $e \bar{e}$ annihilation) at this time is:

$$
n_{e}=\frac{V 4 \pi c m_{e}^{2} k_{b} T_{e} K_{2}(\mu)}{e h^{3}}=\frac{16 \pi^{2} c^{4} t_{e l}^{3} m_{e}^{2} k_{b} T_{e} K_{2}(\mu)}{3 e h^{3}}=2,1700 \times 10^{86}
$$

To estimate the final number of electrons, the respective antiparticle creation and annihilation must be considered. To do so, let us assume that lepton asymmetry prevails according to a proportion of one stable electron created for every $10^{9} e \bar{e}$ annihilations.

For $t_{\Omega}=76,1[\mathrm{~Gy}]:$

$$
\mathrm{M}_{\mathrm{e}}=10^{-9}\left(n_{e} m_{e}+0,8 n_{n} m_{e}\right) \sim 3,55 \times 10^{47}[\mathrm{~kg}]
$$

Finally, the following total mass for the creation of electrons, protons and neutrons is achieved:

$$
\mathrm{M}_{\mathrm{t}}=\mathrm{M}_{\mathrm{p}}+\mathrm{M}_{\mathrm{n}}+\mathrm{M}_{\mathrm{e}}=7,26 \times 10^{50}[\mathrm{~kg}]
$$

The ratio of positive (p) to negative (e) charges is strictly equal to one, since the beta disintegration of a neutron produces one proton and one electron. Therefore, the Maxwell-Juttner relativistic distribution predicts an electrically neutral universe in terms of protons, neutrons and electrons. Based on this relativistic distribution and for a specific cosmological model, the following dynamic temperature-time relation must be met during the proton-electron production process. Indeed, the exact mass ratio is known:

$$
\frac{m_{p(t)}}{m_{e}(t)}=\left[\frac{t_{e l}^{3} T_{e l}}{t_{p r}^{3} T_{p r}}\right]^{1 / 2}=1836,15
$$

Using the above model and equations, along with the Maxwell-Juttner distribution, the dynamic evolution of the model's variables yields a very realistic ratio:

$$
\frac{m_{p(\infty)}}{m_{e}(\infty)}=1837,37
$$




\section{Cosmic neutrinos from SN1987A}

Cosmic neutrino mass can be estimated using the above relation. Indeed, cosmic neutrino mass can be expressed according to proton or electron mass, as:

$$
\frac{m_{v(t)}}{m_{e}(t)}=\left[\frac{t_{e l}^{3} T_{e l}}{t_{v}^{3} T_{v}}\right]^{1 / 2}
$$

The above equation can be developed with the electron temperature equation along with electron creation time. After some manipulations, we get the following expression for cosmic neutrino mass:

$$
m_{v} \sim\left(\frac{k_{b} \sqrt{1-\beta^{2}} m_{e l}^{2} T_{e l} T_{p}}{c^{2} T_{\Omega}}\right)^{1 / 3} \frac{t_{e l}}{t_{\Omega}}
$$

The only undetermined variable in the above equation is the mean $\beta$ of cosmic neutrinos during their creation. The use of $\beta$ is not an easy choice since this particle is still relatively unknown and has three known states (oscillations). Using $\beta^{S N 1987 A}$, estimated from Stodolsky's observations of SN1987A in (Stodolsky, 1988), $(\beta \leq 0,999999998)$, the maximum neutrino mass can be expressed as:

$$
m_{v}^{S N 1987 A} \leq 8,69 \times 10^{-32}[k g]=48,7\left[\mathrm{keVc}^{-2}\right]
$$

While this is too high a mass for electron neutrinos $\left(<2,5\left[\mathrm{eVc}^{-2}\right]\right)$, it fits well for muon neutrinos $\left(\leq 170\left[\mathrm{keV}^{-2}\right]\right)$

In addition, this found value is within the estimated limit of Benes et al, (2005) for the sterile neutrino mass of SN1987A (10-100 [ $\left.\left.\mathrm{keVc}^{-2}\right]\right)$. Also, Bezrukov (2018), from a detailed analysis of the possibilities for the mass of the sterile neutrino, find a value $\sim 3,3\left[\mathrm{keVc}^{-2}\right]$ that it identifies as a possibility that dark matter is made of sterile neutrinos. However, we will see that the amount of neutrino generated cannot explain the abundance of dark matter predicted by the $\Lambda \mathrm{CDM}$ model ( 26\%). 
This maximum mass is situated between that of the electron neutrino and muon neutrino, or:

$$
\begin{gathered}
m_{v_{e}}<m_{v}^{S N 1987 A}<m_{v_{\mu}} \\
2,5 \times 10^{-3}\left[\mathrm{keV}^{-2}\right]<48,7\left[\mathrm{keV}^{-2}\right]<170\left[\mathrm{keV}^{-2}\right]
\end{gathered}
$$

The resulting mass for cosmic neutrinos is $\sim 10$ times lower than that of electrons, and their speed is practically the speed of light c. Of course, cosmic neutrinos can be found to have different masses depending on the assumptions made for $\beta$. The goal here is not to derive precise neutrino mass, which is beyond the scope of this paper. Using the neutrino mass obtained above, the time, temperature, quantity, and total mass of cosmic neutrinos can be achieved using the MaxwellJuttner distribution:

$$
\bar{T}_{v}=\frac{C_{1}}{\left(-t_{v}+b\right)}=\frac{\frac{m_{v} c^{2}}{\sqrt{1-\beta^{2}}}}{k_{b}}=8,9 \times 10^{12}[\mathrm{~K}]
$$

Therefore:

$$
\mathrm{t}_{v}=\mathrm{b}-\frac{k_{b} C_{1}}{\frac{m_{v} c^{2}}{\sqrt{1-\beta^{2}}}}=\frac{t_{\Omega} T_{\Omega}}{T_{p}}+\frac{k_{b} T_{\Omega} t_{\Omega}}{\frac{m_{\nu} c^{2}}{\sqrt{1-\beta^{2}}}}=\left[\frac{T_{\Omega}}{T_{p}}+\frac{k_{b} T_{\Omega}}{\frac{m_{v} c^{2}}{\sqrt{1-\beta^{2}}}}\right] t_{\Omega}
$$

For $t_{\Omega}=76,1[\mathrm{~Gy}]=2,39 \times 10^{18}[\mathrm{~s}]$ and $\beta=0,999999998$, we get:

$$
\mathrm{t}_{v} \sim 7,315 \times 10^{5}[s] \sim 8,4[d] \text { after the beginning }
$$

The neutrino creation potential (without $v \bar{v}$ annihilation) at this time is:

$$
n_{v}=\frac{V 4 \pi c m_{v}^{2} k_{b} T_{v} K_{2}(\mu)}{e h^{3}}=\frac{16 \pi^{2} c^{4} t_{v}^{3} m_{v}^{2} k_{b} T_{v} K_{2}(\mu)}{3 e h^{3}}=3,19 \times 10^{80}
$$

Maximum mass of neutrinos (without annihilation), after a few manipulations for $t_{\Omega}=76,1$ [Gy], is acheived by:

$$
\mathrm{M}_{v}=n_{v} m_{v} \sim 2,77 \times 10^{49}[\mathrm{~kg}]
$$


A conclusion can be made here, neutrino mass (without annihilation) represents a maximum $\sim 4,2 \%$ of proton mass. Based on the model, cosmic neutrino mass cannot explain the origin of the missing mass. Furthermore, based on the Maxwell-Juttner distribution, cosmic neutrinos appeared before electrons, but after baryons. Another way to proceed involves using the known neutrino mass and look at the creation period and predicted mass, but we still get a predicted neutrino mass that is much smaller than that of baryons.

Let us revisit the total predicted mass of $\sim 7 \times 10^{50}$, which is relatively lower (17 to 350 times) than the oft-mentioned total mass of the universe $\left(1,25 \times 10^{52}\right.$ to $\left.2,5 \times 10^{53}\right)$. However, total mass is relative to the age of the universe. Hence, baryon mass could be increased by increasing the age of the universe or by reducing the particle-antiparticle annihilation factor. However, we will see that the so-called missing mass is not that essential to explain galaxy rotation. The mass can be increased, but we will see that the data from the Planck probe give us the mass vs. energy ratio, which allows us to calculate an approximate age of the universe that partly meets the proportions. We will come back to this argument later. With the energy-mass equivalence, when the ratio of total created mass energy to total universe energy at the time of electron production (around the end of the main leptogenesis) is obtained, we get $\beta=0.001$, or a low non-relativistic speed of the baryonic mass, but still within the range of velocity for the MW:

$$
\frac{E_{\text {mass }}}{E_{\text {total }}}=\frac{\frac{M_{t} c^{2}}{\sqrt{1-\beta^{2}}}}{U\left(t_{e l}\right)}=\frac{6,43 \times 10^{67}}{2,72 \times 10^{78}}=2,3 \times 10^{-11}
$$

This energy ratio confirms that the universe, during early leptogenesis, or at the end of the creation of the particles that make up most of the mass, was vastly influenced by radiation (radiation universe) and that the effects associated with mass, such as gravity, were negligible compared to the electromagnetic impact of photon gas. 
Mean total energy of the universe $13,8[\mathrm{~Gy}]$ after the beginning is:

$$
U_{\text {total }} \sim 2,05 \times 10^{69}[\mathrm{~J}]
$$

That energy, when converted to energy-mass equivalence, yields the following mass $(\beta=0,001)$ :

$$
M_{\text {equi-energy }}=\frac{2,0 \times 10^{69} \sqrt{1-\beta^{2}}}{c^{2}}=2,23 \times 10^{52}[\mathrm{~kg}]
$$

The ratio between the baryonic mass and potential energy-mass for the time period $\sim 1$ to 13,8 [Gy], which can be observed by instruments like the Planck probe, would be:

$$
\left[\frac{M_{t}}{M_{\text {equi-energy }}}\right]_{\text {observable }}=\frac{7,26 \times 10^{50} \mathrm{~kg}}{2,23 \times 10^{52} \mathrm{~kg}} \sim 0,032
$$

That energy-matter ratio is smaller than the estimate made from Planck measurements, an estimated $\sim 0,31$ (regular and dark matter). Howerver, that ratio was calculated using the $\Lambda \mathrm{CDM}$ model, which includes dark matter and dark energy as parameters. If dark energy is removed from the equation and only the $\Lambda \mathrm{CDM}$-estimated baryonic mass is considered, the result is closer, or 0,048 .

Let us calculate the mean volumic mass of the universe at the end of proton production:

$$
\rho_{p r}=\frac{M_{p r}}{V}=\frac{7,26 \times 10^{50} \mathrm{~kg}}{\frac{4 \pi}{3} r^{3}}=\frac{7,26 \times 10^{50} \mathrm{~kg}}{\frac{4 \pi}{3}\left(9,39 \times 10^{12}\right)^{3}}=2 \times 10^{11}\left[\mathrm{~kg} \mathrm{~m}{ }^{-3}\right]
$$

Such density is much lower than the approximate density of a proton $\left(\sim 6,7 \times 10^{17}\left[\mathrm{~kg} \mathrm{~m} \mathrm{~m}^{-3}\right]\right)$, showing that the universe could have contained that amount of mass at that time.

We have not yet considered the electrostatic energy associated with protons and electrons. Let us assume that the Coulomb charge was attributed to protons and electrons at the time of baryogenesis and leptogenesis. Indeed, the electrostatic energy of protons and electrons contained in the sphere with a radius of $r_{p r}$ and $r_{e l}$ at the time of protons and electrons is quite significant or, respectively: 


$$
\begin{aligned}
& E_{p r}^{e l}=\frac{3}{5} k_{e} \frac{\left(n_{p r} q_{p r}\right)^{2}}{r_{p r}}=\frac{3}{5} 8,987 \times 10^{9} \frac{\left(3,9 \times 10^{77} \times 1,6021 \times 10^{-19}\right)^{2}}{9,39 \times 10^{12}}=2,24 \times 10^{114}[\mathrm{~J}] \\
& E_{e l}^{e l}=\frac{3}{5} k_{e} \frac{\left(n_{e l} q_{e l}\right)^{2}}{r_{e l}}=\frac{3}{5} 8,987 \times 10^{9} \frac{\left(3,9 \times 10^{77} \times 1,6021 \times 10^{-19}\right)^{2}}{1,72 \times 10^{16}}=1,22 \times 10^{111}[\mathrm{~J}]
\end{aligned}
$$

However, because the quantity of protons, $\mathrm{n}_{\mathrm{pr}}$, and electrons, $\mathrm{n}_{\mathrm{el}}$, created is identical, we get (including neutron disintegration):

$$
\mathrm{n}_{\mathrm{pr}}=\mathrm{n}_{\mathrm{el}}=3,9 \times 10^{77}
$$

Therefore, the total charge becomes neutral, and the potential energy disappears in the aftermath of electron production. However, the electrostatic potential remains active for $\sim 666$ days, which corresponds to the time difference from the appearance of protons and electrons. We will see that the time difference or delay is the cause of a major so-called baryon-free (empty) zone, except for cosmic neutrinos and others neutral particules.

Thus, the actual baryon-photon ratio for the entire universe $(\beta \sim 0,001)$ can be estimated:

$$
\eta_{\mathrm{B}}=\frac{n_{B}}{n_{\gamma}}=\frac{n_{p r}+n_{n}}{6,42 \times 10^{89}}=\frac{4,33 \times 10^{77}}{6,42 \times 10^{89}} \sim 6,7 \times 10^{-13}
$$

A constant value for the age of the universe after baryogenesis, assuming conventional proton and electron half-lives.

This baryon-photon ratio is $\sim 1000$ times smaller that the Bernreuther estimate (2002). This is due to the calculated baryon mass, which is 500 to 1000 times smaller, $\sim 10^{50}[\mathrm{~kg}]$, than the oftsuggested $\sim 10^{53}[\mathrm{~kg}]$. 


\section{Temperature variations in the CMB}

A possible way to address partially the temperature variations in the CMB is found in variations in the energy of the universe during baryogenesis and leptogenesis. Indeed, when protons, neutrons, and electrons were created, a considerable amount of energy was drawn from the photons for the creation of the particles. That one-time energy shift in the early expansion of the universe (0,362 day for the protons and 666 days for the electrons) surely caused a disruption in the photon gas. Moreover, the creation of matter was likely uniform in the volume, but the energy demand may have caused a local disruption over time for the neutrons, and later for the protons and electrons. Let us calculate that energy disruption for the baryons during baryogenesis, relative to the energy of the universe in the pre-baryon era, and for the electrons, relative to the energy of the universe at that time, or ( $\beta$ of protons, electrons $=0,986$ and $\beta$ neutrinos $=$ 0,999999998) :

$$
\begin{aligned}
& \frac{\Delta E_{\text {baryon }}}{\boldsymbol{E}}=\frac{\Delta E_{M_{t}}}{E_{\text {total }}}=\frac{\left(M_{p}+M_{n}\right) c^{2}}{\sqrt{1-\beta^{2}} U\left(t_{p r}\right)}=\frac{1,25 \times 10^{69} \mathrm{~J}}{9.05 \times 10^{81} \mathrm{~J}}=1,38 \times 10^{-13} \\
& \frac{\Delta \boldsymbol{E}_{\text {electron }}}{\boldsymbol{E}}=\frac{\Delta E_{M_{t}}}{E_{\text {total }}}=\frac{M_{e} c^{2}}{\sqrt{1-\beta^{2}} U\left(t_{e l}\right)}=\frac{6,12 \times 10^{65} \mathrm{~J}}{7,81 \times 10^{78} \mathrm{~J}}=9,15 \times 10^{-14} \\
& \frac{\Delta \boldsymbol{E}_{\text {neutrino }}}{\boldsymbol{E}}=\frac{\Delta E_{M_{t}}}{E_{\text {total }}}=\frac{M_{v} c^{2}}{\sqrt{1-\beta^{2}} U\left(t_{v}\right)}=\frac{3,94 \times 10^{70} \mathrm{~J}}{5,0 \times 10^{80} \mathrm{~J}}=5,88 \times 10^{-11}
\end{aligned}
$$

When that energy is put in relation with that of the blackbody, the energy ratio can be expressed in terms of temperature as:

$$
\begin{gathered}
\frac{\Delta T_{\text {baryon }}}{T}=\left[\frac{\Delta E_{\text {baryon }}}{E}\right]^{1 / 4}=\left(1,38 \times 10^{-13}\right)^{1 / 4} \sim 6,1 \times 10^{-4} \\
\frac{\Delta T_{\text {electron }}}{T}=\left[\frac{\Delta E_{\text {electron }}}{E}\right]^{1 / 4}=\left(9,15 \times 10^{-14}\right)^{1 / 4} \sim 5,5 \times 10^{-4}
\end{gathered}
$$




$$
\frac{\Delta T_{\text {neutrino }}}{T}=\left[\frac{\Delta E_{\text {neutrino }}}{E}\right]^{1 / 4}=\left(5,88 \times 10^{-11}\right)^{1 / 4} \sim 3 \times 10^{-3}
$$

Following measurements made by Planck, the analysis and explanation of temperature variations in the CMB became priorities. Ever since the initial analyses and Fixsen's synthesis (Fixsen, 2009), assessments of temperature variations in the CMB continually varied as new interpretations were made and instruments were perfected. Variations sit within a range of values put forth by separate authors. Without going into finer detail, the range of values is as follows:

Planck, $2015 \quad$ Fixsen, 2009

$$
\begin{gathered}
{\left[\frac{ \pm 27 m K}{2,722 K}\right]<\left[\frac{\Delta T}{T}\right]_{\text {exp }}<\left[\frac{ \pm 570 \mu K}{2,72548 K}\right]} \\
\pm 9,9 \times 10^{-3}<\left[\frac{\Delta T}{T}\right]_{\text {exp }}< \pm 2,1 \times 10^{-4}
\end{gathered}
$$

This shows that baryogenesis and leptogenesis, or variation of energy for the creation of protons, electrons and neutrinos, is in the order of magnitude of the overall temperature variations in the CMB (energy disruption or negative energy jump of the photons during the creation of matter). Could those temperature variations in the CMB be partially caused by successive energy jumps during particle creation, in addition to the vibrational mode of baryons (Eisenstein, Zehavi, Hogg et al., 2005) ? Moreover, analyses of the variations do not seem to show any anisotropy, except for great empty zones. This supports the notion of isotropic energy variations for the entire volume that is compatible with the creation of a uniform mass in the volume. Finally, because protons, neutrons and electrons, and the particle fusion cycles, occurred at different times and different energy levels for the photons in the photon gas, notable variations $(\Delta T / T)_{i}$ could be found in the variations of energy spectrum of the CMB in line with the energy levels successively implicated 
in beryogenesis and leptogenesis, and at successive times for the protons-neutrons, electrons, deuterium, etc.

\section{Expanding 3d-sphere of matter}

An order of magnitude for the avarage speed of baryonic matter can be calculated with a theoretical mean mass density of the universe, the Hubble-Lemaitre expansion law, the cosmic time and the assumption that the boundary of the universe is moving constantly at the speed of light.

Let us suppose that this sphere of matter was at state 1 at the time of early creation of great structures like galaxies $(<2[\mathrm{~Gy}])$, whose boundaries were expanding at the speed of light towards state 2 , or the current age of the universe, written as $t_{\Omega}$. Let us also suppose a material point in the sphere in state 1 (e.g. the original bulbe of matter at the center of the MW), which undergoes expansion until today. That point is not located at the mathematical centre of the sphere, but at a given location written as $r_{1}$ at state 1 . The material point evolves towards a material position 2 in state 2 , moving at a mean speed $\bar{\beta}$ (non-relativist). Moreover, considering expansion and displacement at the mean speed in the direction of expansion, the following equation yields the position of the material point at state 1 at time $t_{0}$ in the sphere of matter at the time of state 2 (universe age $t_{\Omega}$ ):

$$
t_{0}=\frac{t_{\Omega}}{t_{1}} \frac{r_{1}}{c}+\frac{t_{1}}{t_{\Omega}} \bar{\beta}\left(t_{\Omega}-t_{1}\right)=t_{\Omega} \frac{r_{1}}{R_{1}}+\frac{t_{1}}{t_{\Omega}} \bar{\beta}\left(t_{\Omega}-t_{1}\right)
$$

Where: $\quad R_{1}=c t_{1} ; R_{\Omega}=R_{2}=c t_{\Omega}$

The first term is the expansion of the material point in the expanding volume during the time period, and the second term is the effect of the speed modulated by the inverse of expansion. The equation has four mathematically independent variables that must be compatible from a physics standpoint. 
Indeed, for each quartet $\left(r_{1}, t_{1}, t_{\Omega}, \bar{\beta}\right)$, the value of $t_{0}$ must be lower than or equal to $t_{\Omega}$, which limits possibilities, or still, forces a restriction on variable $\bar{\beta}$. In this paper, we only consider the mean value of $\bar{\beta}$ for a sphere of matter undergoing Hubble-Lemaître expansion, the boundary of which is moving at $\beta=1$. The cosmological principle states, at least, that there are no preferred positions. However, expansion of the universe occurs in a precise order of events, each appearing at its own cosmic time, which leads to the idea that for a much larger universe than what we can observe today, one can imagine relative positions within that chronological universe. Moving forward with that idea, one can estimate an approximate position for the MW in the sphere universe. Indeed, we will see in the next section, dealing with a mass rotation model for a few galaxies with the combined action of gravitational force and cosmological gravity, that initial formation of the MW could have started around 150-190 [My] after the beginning, and that main formation could have taken 380-450 [My]. Therefore, let us start with a sphere universe of state 1 at time 1 [Gy] $\left(\mathrm{t}_{1}=1[G y]\right)$, that is a sphere of matter that is large enough to contain the MW bulbe. Initial formation of the bulbe yields $r_{1} /_{R_{1}}=0,15-0,19[\mathrm{~Gy}] /[G y]$. Moreover, by selecting $\bar{\beta}$ according to an equation developed in the next section $\left(\bar{\beta} \sim 2 \times 10^{-3}\right)$, and $t_{0}$, the age of the universe calculated by Planck (13,8 [Gy]) at our observation position, we get an approximate range of ages for the universe today:

$$
t_{\Omega} \sim 73 \text { to } 92[\mathrm{~Gy}]
$$

That number must be seen as sufficient to create the required energy for the universe to generate a baryonic mass that is close to the mass estimated from observations of the cosmos, while providing a possible explanation for the formation periods and rotations of the galaxies being studied. 


\section{Pressure in the CMB and the Casimir effect: A possible age of the universe}

The Casimir Effect is often used to explain what authors call vacuum energy or vacuum force. There is a model we can use to further analyze this effect and see if it can be partially explained and provide useful information.

Readers can refer to numerous works on the Casimir Effect and its electromagnetic origin (Kawka, 2010). If the Casimir force is expressed as shown in works where parallel plates are used, we get the following equation:

$$
\mathrm{F}_{\mathrm{c}}=\left(\frac{\pi^{2}}{240}\right) \frac{h c}{2 \pi l^{4}} \mathrm{~S}
$$

Where $l$ represents the distance between the parallel conductive plates, and $\mathrm{S}$ is the surface of the plates. The constant is obtained from the integration of potential photon vibration modes between the plates (the space between the plates act as a resonant cavity for the photons). This normally attractive force can be expressed as radiation pressure:

$$
\mathrm{F}_{\mathrm{c}}=P_{C} \mathrm{~S}
$$

The quantities of energy in the universe on a per-era basis are known, which can be expressed in the form of mean density of energy in the volume, as:

$$
\mathrm{F}_{\mathrm{c}}=\left(\frac{U(t)}{V(t)}\right) \mathrm{S}
$$

From the photon gas energy expression, an expression of Casimir force, from a standpoint of properties at time $t$, is written as:

$$
\mathrm{F}_{\mathrm{c}}(\mathrm{t})=\left(\frac{N h \vartheta}{V}\right) \mathrm{S}=\left(\frac{2 \pi N h c}{2 \pi V \lambda}\right) \mathrm{S}=\left(\frac{2 \pi N(t)}{V(t) \lambda(t)}\right) \frac{h}{2 \pi} c \mathrm{~S}
$$


Where $\mathrm{N}$ is the constant number of photons after the photon inflation period, or about $10^{-13}[\mathrm{~s}]$ $\left(\mathrm{N} \sim 6,4 \times 10^{89}\right)$. Moreover, if we postulate that Casimir pressure is generated by CMB photons at our position $\mathrm{t}_{0}$, then:

$$
\mathrm{F}_{\mathrm{c}}=\mathrm{P}_{\mathrm{c}} \mathrm{S}=\left(\frac{2 \pi N(\infty)}{V_{0} \lambda_{c m b}}\right) \frac{h}{2 \pi} c \mathrm{~S}
$$

The above Casimir Effect equation makes it possible to calculate pressure at time $t_{0}$ (at our position in the universe) when the mean wavelength of photons in the CMB is known. As with CMB temperature, Casimir pressure is an observable property of the universe. That wavelength is well known and derived from the Wien's law, as:

$$
\lambda_{\mathrm{cmb}}=\frac{\sigma_{w}}{T_{c m b}}=\frac{2,89777 \times 10^{-3}}{2,728}=1.06 \times 10^{-3}[\mathrm{~m}]
$$

In a manner of speaking, that pressure is the same as theoretical pressure in a vacuum (CMB radiation pressure), considering the fact the energy of the universe decreased when the particles were created. To determine that pressure, we could estimate the position of the observer, $\mathrm{t}_{1}$, in the universe. To do so, we know the expression for photon gas pressure at the same time, $t_{1}$, and we get the following expression to determine a possible position in the universe or cosmic time:

$$
\begin{gathered}
\mathrm{P}_{\mathrm{c}}=\mathrm{P}_{\text {gas } \gamma} \\
\left(\frac{2 \pi N(\infty)}{V_{1} \lambda_{f d c}^{1}}\right) \frac{h}{2 \pi} c=\frac{\zeta(4) N(\infty) k_{b} T_{1}}{\zeta(3) V_{1}} \\
\left(\frac{h c}{\lambda_{c m b}^{1}}\right) \sim \frac{\zeta(4)}{\zeta(3)} k_{b} T_{1}
\end{gathered}
$$




$$
h c=\frac{\zeta(4)}{\zeta(3)} k_{b} T_{1} \lambda_{c m b}^{1}
$$

The wavelength of the CMB, as perceived by an observer at point $t_{1}$, is not modified by the scale factor:

$$
\lambda_{c m b}^{1}=\lambda_{c m b}(\text { constant })
$$

Then with the temperature equation:

$$
h c=\frac{\zeta(4)}{\zeta(3)} k_{b} \lambda_{c m b} T_{1}=\frac{\zeta(4)}{\zeta(3)} k_{b} \frac{\sigma_{w}}{T_{\Omega}} T_{1}=\frac{\zeta(4)}{\zeta(3)} k_{b} \sigma_{w} \frac{t_{\Omega}}{t_{1}}
$$

Or with the expression $\sigma_{w}$ using the Lambert function:

$$
\frac{t_{1}}{t_{\Omega}}=\frac{\zeta(4)}{\zeta(3)} \frac{k_{b} \sigma_{w}}{h c}=\frac{\zeta(4)}{\zeta(3)} \frac{k_{b} h c}{\left(5+W_{0}\left(-5 e^{-5}\right)\right) k_{b} h c}=\frac{\zeta(4)}{\zeta(3)} \frac{1}{\left(5+W_{0}\left(-5 e^{-5}\right)\right)} \sim \frac{0,9004}{4,9651} \sim 0,18134
$$

In the above equation, if we assume that the position of the MW is 13,8 [Gy] $\left(\mathrm{t}_{1}=\mathrm{t}_{0}\right.$ observable universe at our position), a possible cosmic age of the universe would be 76,098 [Gy] ( 76,1 [Gy]). This is a surprising result, as it implies that the following ratio of physics constants is relative to position in the universe, or:

$$
\frac{k_{b} \sigma_{w}}{h c}=\frac{\zeta(3)}{\zeta(4)} \frac{t}{t_{\Omega}}
$$

Of course, if that equation holds true, its cosmological implications are important. The equation can be rewritten assuming that Wien's law is universal and that the speed of light for photons is always the product of wavelength times frequency, or: 


$$
\frac{k_{b}}{h}=\left(\frac{c}{\sigma_{w}}\right) \frac{\zeta(3)}{\zeta(4)} \frac{t}{t_{\Omega}}=\left(\frac{\lambda v}{\lambda T}\right) \frac{\zeta(3)}{\zeta(4)} \frac{t}{t_{\Omega}}=\left(\frac{v}{T}\right) \frac{\zeta(3)}{\zeta(4)} \frac{t}{t_{\Omega}}
$$

The ratio of $v$-origin photon frequency to temperature $T$ is strictly constant $\left(1,034 \times 10^{11}\left[\mathrm{~s}^{-1} \mathrm{~K}^{-1}\right]\right)$ from the initial Planck time $t_{p}$ up to 76,1 [Gy]. Finally, we get:

$$
\frac{k_{b}}{h}=k f\left(\frac{t}{t_{\Omega}}\right) \text { (function of position in the universe or cosmic time) }
$$

The implications of that equation are beyond the scope of this paper. The previous section, Expanding 3d-sphere of matter, we arrived at the following expression, which we equate to the result we obtained for $t_{0}$ :

$$
\frac{t_{0}}{t_{\Omega}}=\frac{r_{1}}{R_{1}}+\frac{t_{1}}{t_{\Omega}^{2}} \bar{\beta}\left(t_{\Omega}-t_{1}\right) \sim 0,18134
$$

This constant ratio is surprising! It implies that mass speed increases with time as the universe ages, in order to conserve a quasi constant quotient for a given structure (or a given position, $\mathrm{t}_{1}$ ). In other words, using the MW as an example, its speed would appear to increase with the increase in the age of the universe. Therefore, for a sphere of matter beginning at 1 [Gy], we use the following to determine the speed of the MW at $t_{0}\left(13,8[\mathrm{~Gy}]\right.$ and $r_{1} / R_{1}$ assumed to be 0,181314 in the $1[\mathrm{~Gy}]$ sphere to derive the speed of the MW today):

$$
\begin{gathered}
\beta\left(t_{0}\right)=\frac{v}{c}=\frac{\dot{r}}{c}=\frac{\frac{t_{\Omega}^{2}}{t_{1}}\left(\frac{t_{0}}{t_{\Omega}}-\frac{r_{1}}{R_{1}}\right)}{\left(t_{\Omega}-t_{1}\right)}=\frac{\frac{76,1^{2}}{1}(0,181340-0,181314)}{(76,1-1)}=2,004 \times 10^{-3} \\
\text { Or } v_{m w} \sim 600\left[\mathrm{~km} \mathrm{~s}^{-1}\right]
\end{gathered}
$$

The following three figures $(8,9 \& 10)$ show the form of that evolving speed, or $v=$ $\beta c$, acceleration, $a$, and the intrinsic deceleration factor, $\mathrm{q}$, of the $\mathrm{MW}$ relative to the age of the 
universe for a sphere of matter starting at $1[\mathrm{~Gy}]$ and expanding. The MW is at position $\sim 0,181314$ [Gy] in that sphere (start of bulbe formation). We use 1 [Gy] sphere because the MW started to expand after its creation, or an initial sphere larger than 181 [My]. Note that the speed of the MW today is an estimated $\sim 600\left[\mathrm{~km} \mathrm{~s}^{-1}\right]$. That value for the current speed of the MW corresponds relatively well with the estimates made by Kraan-Korteweg et al. (1998).

As for acceleration, we find a very reliable number, which is nevertheless not zero:

$$
a_{m w}=\dot{v}_{m w}=\frac{d v_{m w}}{d t_{\Omega}}=\frac{\frac{c t_{\Omega}^{2}}{t_{1}}\left(\frac{t_{0}}{t_{\Omega}}-\frac{r_{1}}{R_{1}}\right)-2 c \frac{t_{\Omega} t_{1}}{t_{1}}\left(\frac{t_{0}}{t_{\Omega}}-\frac{r_{1}}{R_{1}}\right)}{\left(t_{\Omega}-t_{1}\right)^{2}}
$$

In brief, the MW was moving slowly in the direction of the beginning (closed universe) after principal formation up to $\sim 2[\mathrm{~Gy}]$. Then, expansion of the mass began, and the MW started to accelerate towards the boundary (open universe). Also, the variation of acceleration, $\dot{a}$, is slightly positive $\left(\sim 1 \times 10^{-33}\left[\mathrm{~m} \mathrm{~s}^{-3}\right]\right.$ at $\left.\mathrm{t}_{0}\right)$, showing that the mass accelerates in the direction of expansion.

Finally, for an intrinsic deceleration factor, we get the following expression, which is based on the conventional definition. Moreover, it should be noted that in this version of the model, the deceleration factor, q, of the boundary of the universe is zero, as it moves at constant speed c. However, mass in the volume of the universe is moving with a negative deceleration factor (acceleration). This is an important difference because the observation of motion in supernovas does not automatically guarantee that such motion applies without distinction at the boundary of the universe. For the deceleration factor of a given mass (intrinsic) we get (based on the definition of $q)$ :

$$
q_{m}=\frac{-\ddot{r}_{m} r_{m}}{\dot{r}_{m}^{2}}=\frac{-\ddot{r}_{m}}{\dot{r}_{m}^{2}} \frac{\dot{r}_{m}}{H}=\frac{-a_{m}}{\dot{r}_{m} H}=\frac{-a_{m} t}{\dot{r}_{m}}
$$




$$
q_{m}=-t \frac{c \frac{\frac{t_{\Omega}^{2}}{t_{1}}\left(\frac{t_{0}}{t_{\Omega}}-\frac{r_{1}}{R_{1}}\right)}{\left(t_{\Omega}-t_{1}\right)^{2}}-2 c \frac{\frac{t_{\Omega} t_{1}}{t_{1}}\left(\frac{t_{0}}{t_{\Omega}}-\frac{r_{1}}{R_{1}}\right)}{\left(t_{\Omega}-t_{1}\right)^{2}}}{c \frac{t_{\Omega}^{2}}{t_{1}}\left(\frac{t_{0}}{t_{\Omega}}-\frac{r_{1}}{R_{1}}\right)}=\frac{2 t_{1}-t}{t-t_{1}}
$$

It is apparent here that the deceleration factor tends towards -1 as the age of the universe increases. This means that expansion is constantly accelerating and the universe is open. Here, $t_{1}$ is understood to be the starting value (sphere) of the expansion factor computation, or after the initial formation of the great structures (1-2 [Gy]). The deceleration factor, $q_{m}(\mathrm{z})$, can be obtained either according to the relative distance to the MW, or to $\mathrm{z}$, the relative cosmological redshift to the MW:

$$
Z=\frac{a_{0}}{a}-1=\frac{r_{0}}{r}-1=\frac{t_{0}}{t}-1
$$

By substituting the expression for $\mathrm{z}$ in $\mathrm{q}$, the following equation for the deceleration factor is achieved:

$$
q_{m}(z)=\frac{2 t_{1}(z+1)-t_{0}}{t_{0}-t_{1}(z+1)}
$$

Where $t_{0}=13,8[\mathrm{~Gy}]$ and $t_{1}=1[\mathrm{~Gy}]$, then:

$$
q_{m}(z)=\frac{z+1}{12,8-z}-1
$$

Figures 10 and 11 show deceleration factors $\mathrm{q}_{\mathrm{m}}(\mathrm{t})$ and $\mathrm{q}_{\mathrm{m}}(\mathrm{z})$. Based on the resulting curves, it can be seen that at the beginning of expansion, the universe, or the mass, decelerated to $z_{t}>5,9$ ( $\mathrm{t} \sim 2$ [Gy]). Then, the mass accelerated. Measurements by Reiss et al. (1998) and Kiselev (2003) are shown on the curves. Therefore, the model seems to perform rather well in terms of deriving values of $\mathrm{q}$ for the low values of $\mathrm{z}$. However, the model predicts a deceleration-acceleration transition 
earlier than most other predictive models for $\mathrm{q}(\mathrm{z})$. For comparison purposes, $z_{t}$ is closer to 0,7 according to Giostri et al (2012), who used a calibrated parametrical model with a prescribed constant of $q(z)=1 / 2$ for $t \rightarrow 0$. That prescribed value is in fact being questioned by researchers. Based on the model, the deceleration of mass in the universe is quite substantial. Then, after $\sim 2$ [Gy], expansion starts to increase, and the mass accelerates in small steps.

In the above equation, if the age of the universe is assumed to be 76,1 [Gy], then $q=-0,986$.

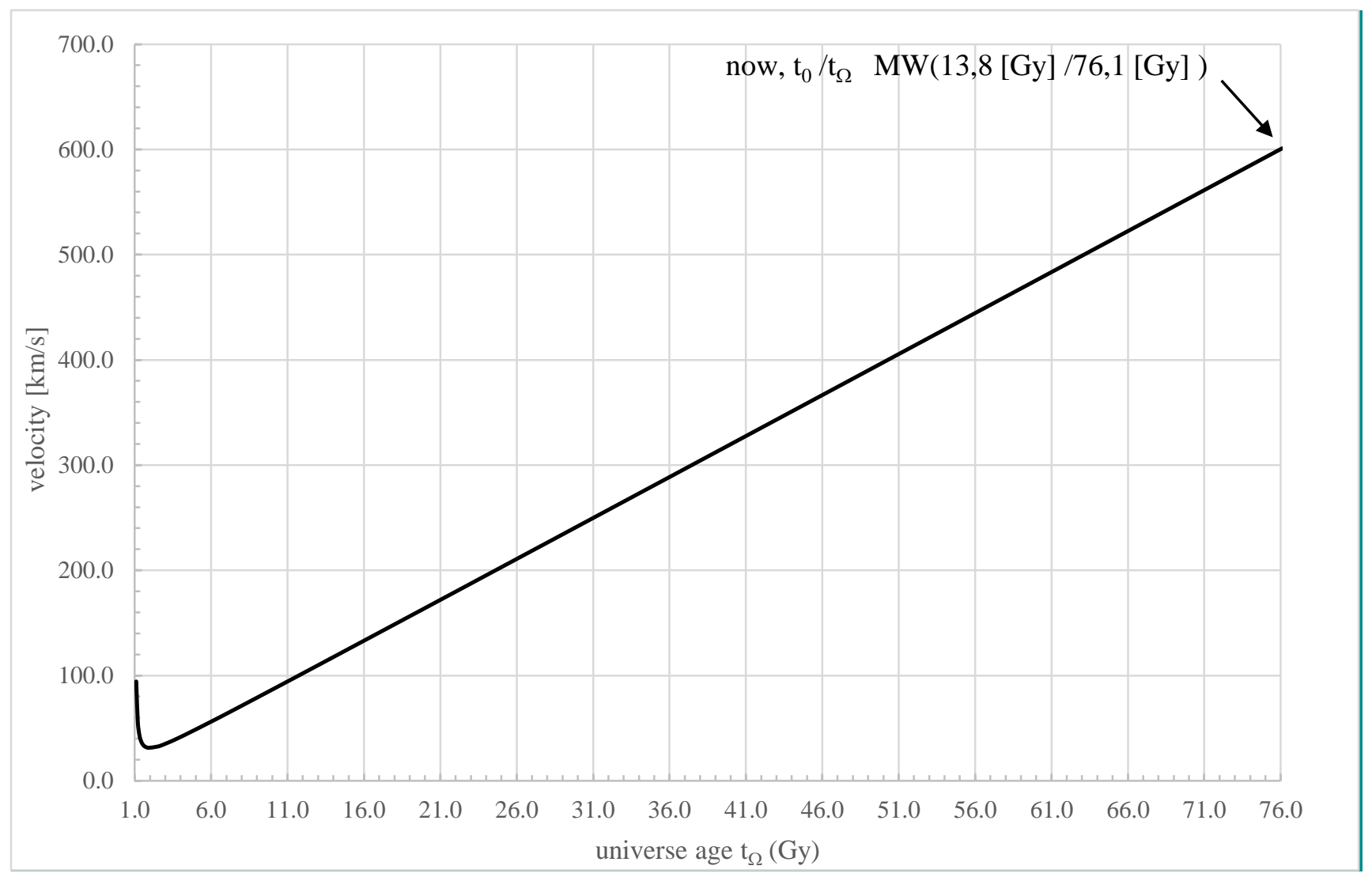

Figure 8: MW intrinsic velocity for $\mathrm{t}_{\Omega}=1[\mathrm{~Gy}]$ to $76,1[\mathrm{~Gy}]$ 


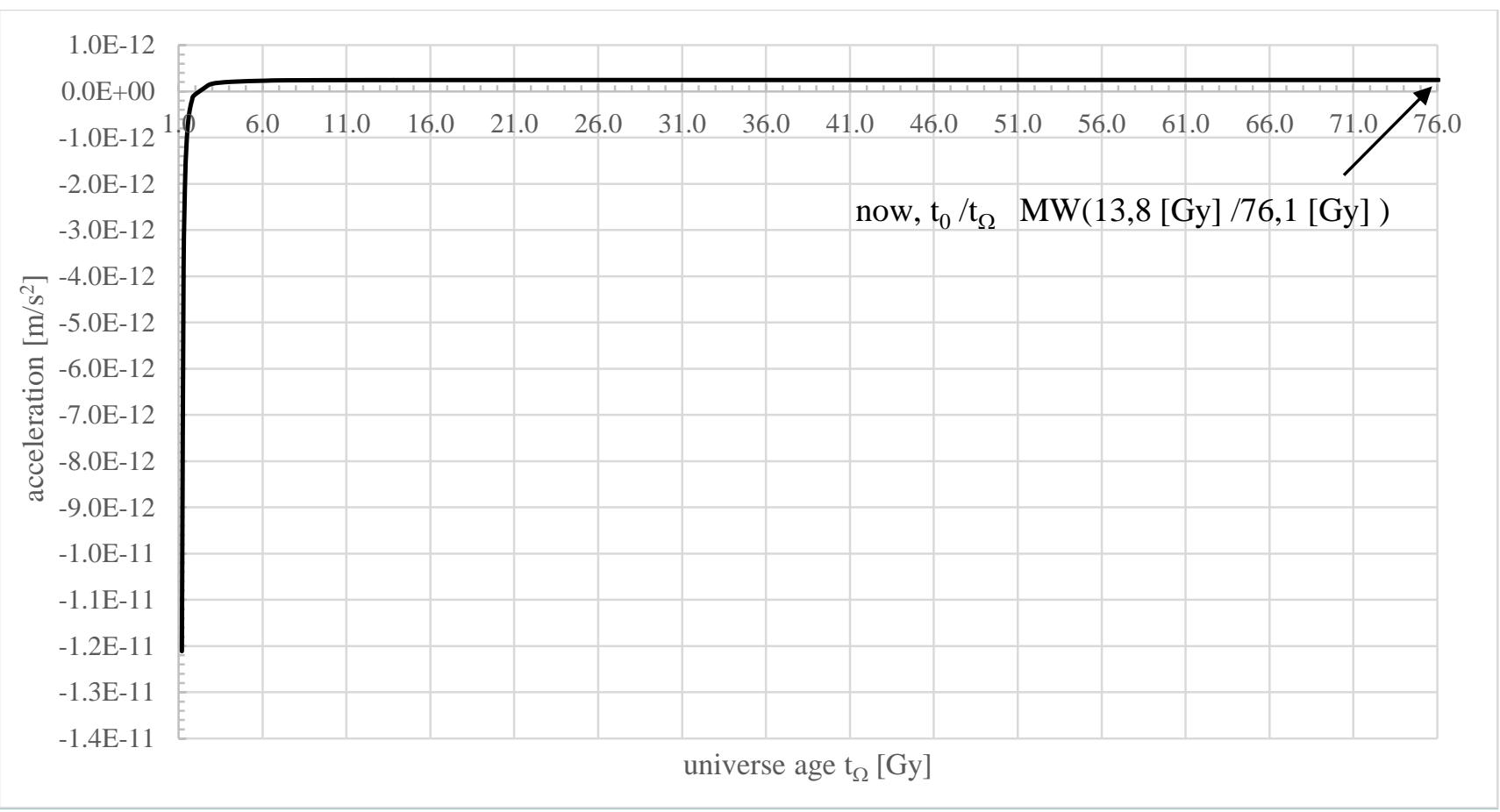

Figure 9a: MW intrinsic acceleration for $\mathrm{t}_{\Omega}=1[\mathrm{~Gy}]$ to $76,1[\mathrm{~Gy}]$ 


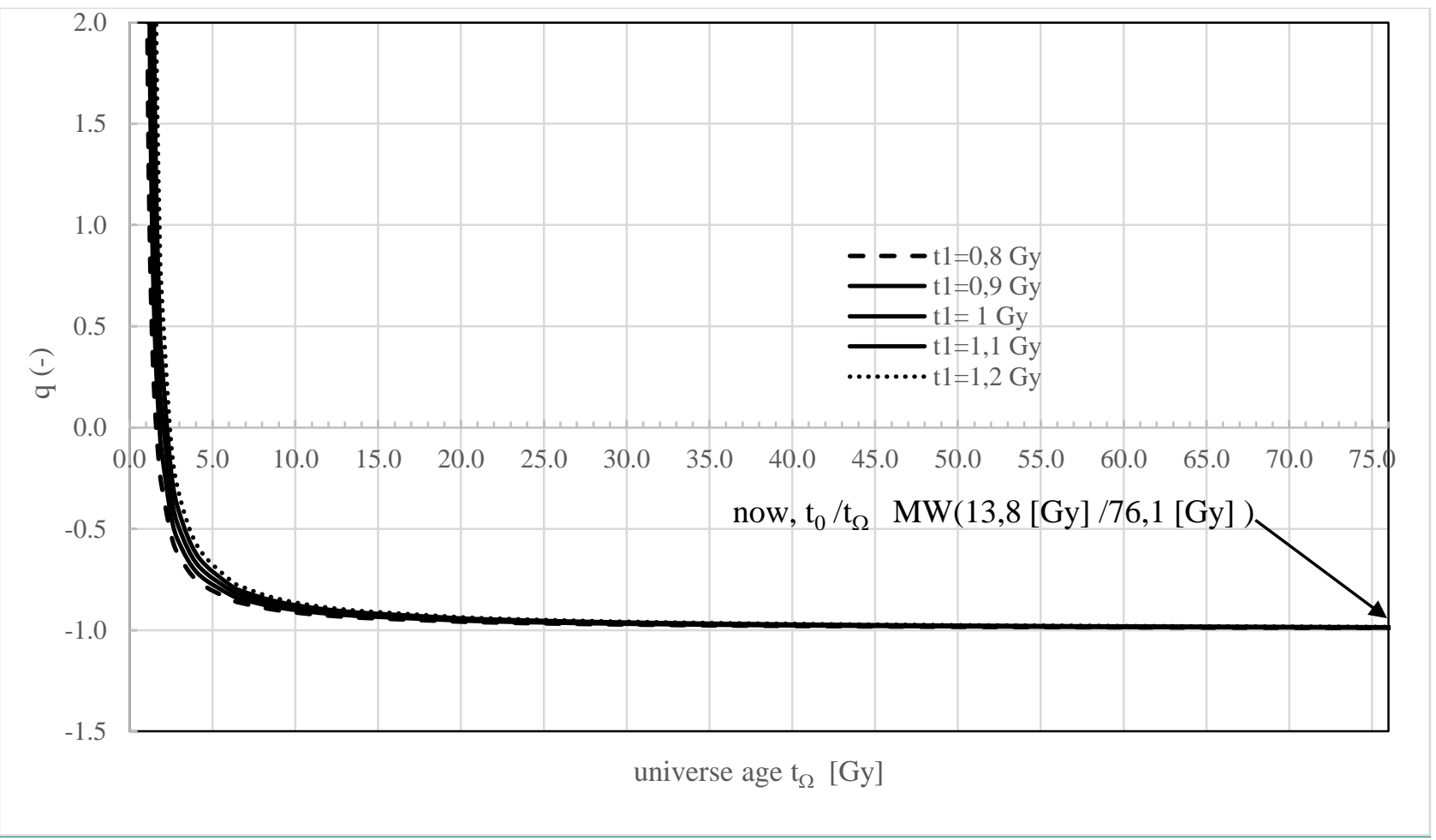

Figure 10: MW intrinsic deceleration parameter for $\mathrm{t}_{\Omega}=1[\mathrm{~Gy}]$ to $76,1[\mathrm{~Gy}]$

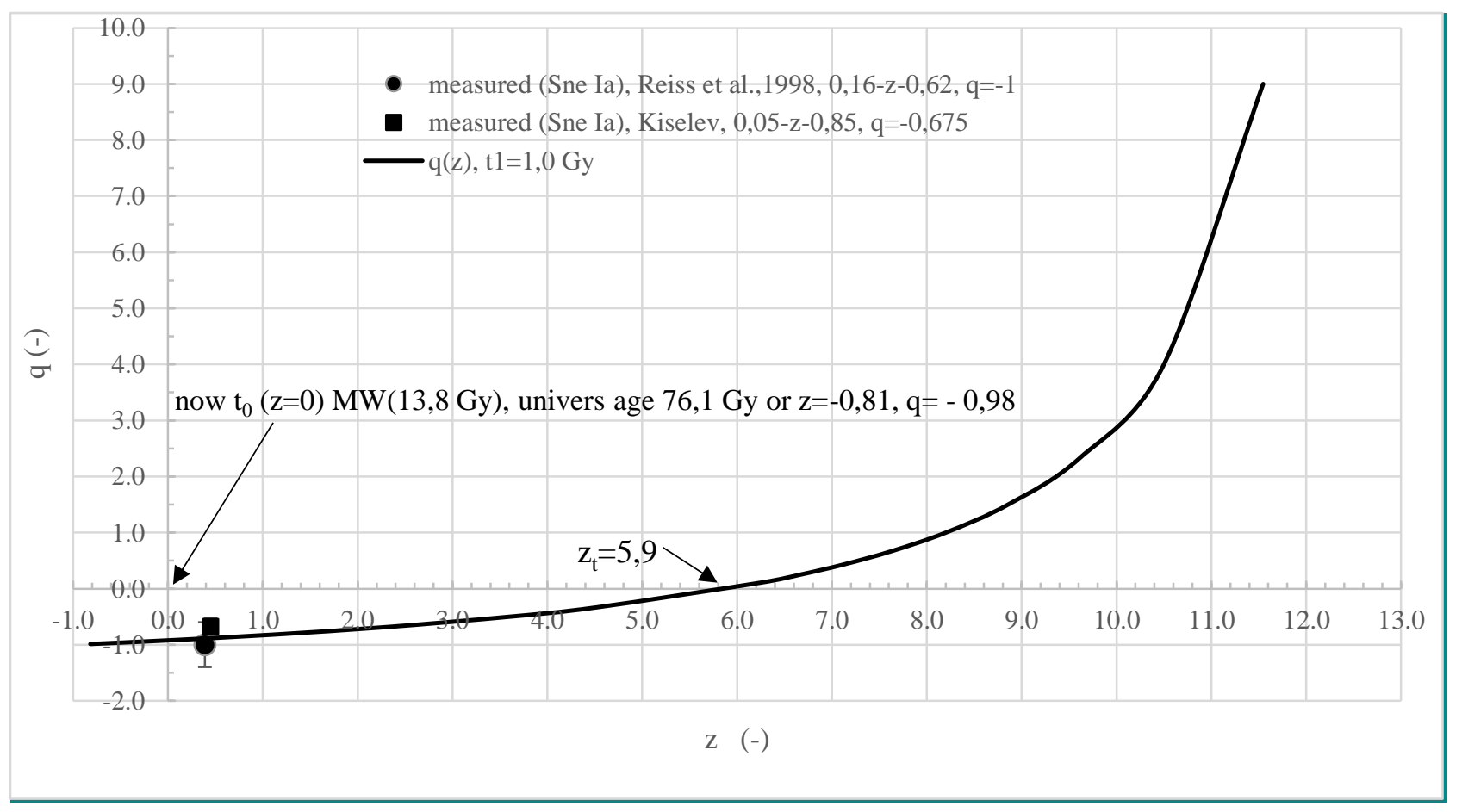

Figure 11: Masses deceleration parameter function of $z$ 
If we develop the above equation in terms of the Hubble-Lemaitre expression, or from the beginning $r_{1}=0$ until $t_{\Omega}$, noting that $t_{0}=t_{1}=0, \dot{r}=c \beta$ and $r=c t_{\Omega}$ correspond to the speed of expansion and observed distance, and if $t_{\Omega}-t_{1}=t$, then:

$$
\beta=\frac{v}{c}=\frac{\dot{r}}{c}=\frac{\frac{t_{\Omega}^{2}}{t_{1}}\left(\frac{t_{0}}{t_{\Omega}}\right)}{\left(t_{\Omega}-t_{1}\right)}=\frac{t_{\Omega}}{t}
$$

Or:

$$
\dot{r}=\left(\frac{1}{t}\right) r=H r
$$

Which is in fact the Hubble-Lemaitre expression as observed from our viewpoint, with $H=\frac{1}{t}$. However, it should be noted that validation of the Hubble-Lemaître law principally comes from the observation of galaxies, a period of the existing universe after their formation, around 0,1 to 2 [Gy], or the expansion of a sphere at time $t_{1}$ towards another sphere at time $t_{2}$, and not from a dimensionless starting point towards a sphere. This is an important detail because it puts into perspective the fact that the Hubble-Lemaitre law is experimental, resulting from the observation of great structures over a period of time which logically begins when those structures have already been formed.

Let us return to Casimir pressure which, relative to $z$, is:

$$
\begin{gathered}
\mathrm{P}_{c} \sim\left(\frac{2 \pi N(\infty)}{V_{1} \lambda_{f d c}^{1}}\right) \frac{h}{2 \pi} c=16 \pi\left(5+W_{0}\left(-5 e^{-5}\right)\right) \xi(3) \frac{k_{b} T_{f d c}(z+1)^{3}}{\lambda_{f d c}^{3}} \sim 16 \pi(4,965)(1,202) \frac{k_{b} T_{f d c}(z+1)^{3}}{\lambda_{f d c}^{3}} \sim 1,291 \times 10^{-11}(z+1)^{3} \\
P_{c}=P_{c}^{0}(z+1)^{3}
\end{gathered}
$$

Based on this approach, such minimum or zero Casimir energy pressure, $P_{c}^{0}$, would be lower than what can be obtained from our position in the universe, and only corresponds to the pressure found 
with the original photons and no matter. This may correspond to the volumic energy state from point zero to our position. Today, pressures as low as $\sim 10^{-10}$, or extreme vacuum, have been measured at (Conseil Européen pour la Recherche Nucléaire, 2018). Expressing that pressure in terms of amplified pressure between two parallel reflecting plates at distance $l$ from each other (cavity), the maximum distance required to arrive at that minimum pressure is in the order of 0,1 $[\mathrm{mm}]$, or:

$$
l_{\max }=\left(\frac{\pi h c}{480 P_{c}^{0}}\right)^{\frac{1}{4}} \sim 1,001 \times 10^{-4}[\mathrm{~m}]
$$

To see if that minimum pressure corresponds closely with experimental results designed to determine whether the theoretical value obtained for that pressure is in the order of magnitude of the estimated pressure. Decca et al. (2007) tested the Casimir effect using a torsion oscillator between two gold-coated parallel plates. The smallest pressure mentioned is in the order of $3[\mathrm{mPa}]$, or one billion times greater than the minimum pressure obtained, $P_{c}^{0}$. They reported the following measurements (table 1):

Table 1: Measured length and Casimir pressure by Decca, 2007

\begin{tabular}{ccc}
$\boldsymbol{l}[\boldsymbol{n m}]$ & $\boldsymbol{l}^{\mathbf{4}}\left[\mathbf{n m}^{\mathbf{4}}\right]$ & $\boldsymbol{P}_{\boldsymbol{c}}^{\boldsymbol{e x p}}[\boldsymbol{P a}]$ \\
$1,6500000000 \mathrm{E}-07$ & $7,4120062500 \mathrm{E}-28$ & $1,0200000000 \mathrm{E}+00$ \\
\hline $2,0000000000 \mathrm{E}-07$ & $1,6000000000 \mathrm{E}-27$ & $4,9000000000 \mathrm{E}-01$ \\
\hline $3,0000000000 \mathrm{E}-07$ & $8,1000000000 \mathrm{E}-27$ & $1,10 \mathrm{E}-01$ \\
\hline $4,0000000000 \mathrm{E}-07$ & $2,5600000000 \mathrm{E}-26$ & $3,35 \mathrm{E}-02$ \\
\hline $5,0000000000 \mathrm{E}-07$ & $6,2500000000 \mathrm{E}-26$ & $1,55 \mathrm{E}-02$ \\
\hline $6,0000000000 \mathrm{E}-07$ & $1,2960000000 \mathrm{E}-25$ & 0,0075 \\
\hline $7,4000000000 \mathrm{E}-07$ & $2,9986576000 \mathrm{E}-25$ & $3,20 \mathrm{E}-03$
\end{tabular}

An empiric correlation can be obtained from the data with the following equation: 


$$
P_{c}^{e x p} \sim \frac{1 \times 10^{-26}}{\left(l^{4}\right)^{0,957}}
$$

If we estimate the minimum pressure predicted in the above correlation with the maximum $l$ value $\left(1,001 \times 10^{-4}[\mathrm{~m}]\right)$, then:

$$
P_{C}^{\min } \sim \frac{1 \times 10^{-26}}{\left(l_{\max }^{4}\right)^{0,957}} \sim 2,038 \times 10^{-11}[\mathrm{~Pa}]
$$

That number is very close to the estimated minimum Casimir pressure, or the following ratio, which does not indicate the existence of a minimum pressure for a maximum value of $l$ in the experiments by Decca et al. However, if this minimum truly exists, the result of those experiments would yield a result in the order of magnitude of the predicted value, or:

$$
\frac{P_{c}^{\min }}{P_{c}^{0}} \sim \frac{2,038 \times 10-11}{1,291 \times 10-11} \sim 1,58
$$

By using the Casimir Effect, we get amplification of that pressure by photon resonance in the CMB in the different experimental setups and, in particular, in the cavity between the reflecting plates. That amplification can be expressed as:

$$
P_{c}=\eta P_{c}^{0}
$$

Where

$$
\eta=\frac{\left(\frac{\pi^{2}}{240}\right) \frac{1}{l^{4}}}{\frac{2 \pi N(\infty)}{V_{0} \lambda c m b}}=\left(\frac{\pi^{2}}{240}\right) \frac{V_{0} \lambda_{f d c}}{2 \pi N(\infty) l^{4}}=\frac{1.006 \times 10^{-16}}{l^{4}}
$$

And for P: 


$$
P_{c}=\eta P_{C}^{0}=\frac{1,006 \times 10^{-16}}{l^{4}} 1,291 \times 10^{-11}=\frac{1,3001 \times 10^{-27}}{l^{4}}
$$

The theoretical coefficient is equal to $1,3001 \times 10^{-27}$. The experimental coefficient found by Bressi et al. (2002) is $1,22 \pm 0,18 \times 10^{-27}$. For a typical value of $1=200[\mathrm{~nm}]$, the minimum Casimir pressure is amplified by $\eta \sim 6,3 \times 10^{10}$. Based on this model, the maximum scope of the $1_{\max }$ Casimir Effect between two plates is $\sim 0,1[\mathrm{~mm}]$, because at any greater distance the pressure would be below the minimum value of $P_{c m b}^{0}$ at our position in the universe. Figure 12 shows the Casimir zero pressure and the photon gas pressure relative to the age of the universe.

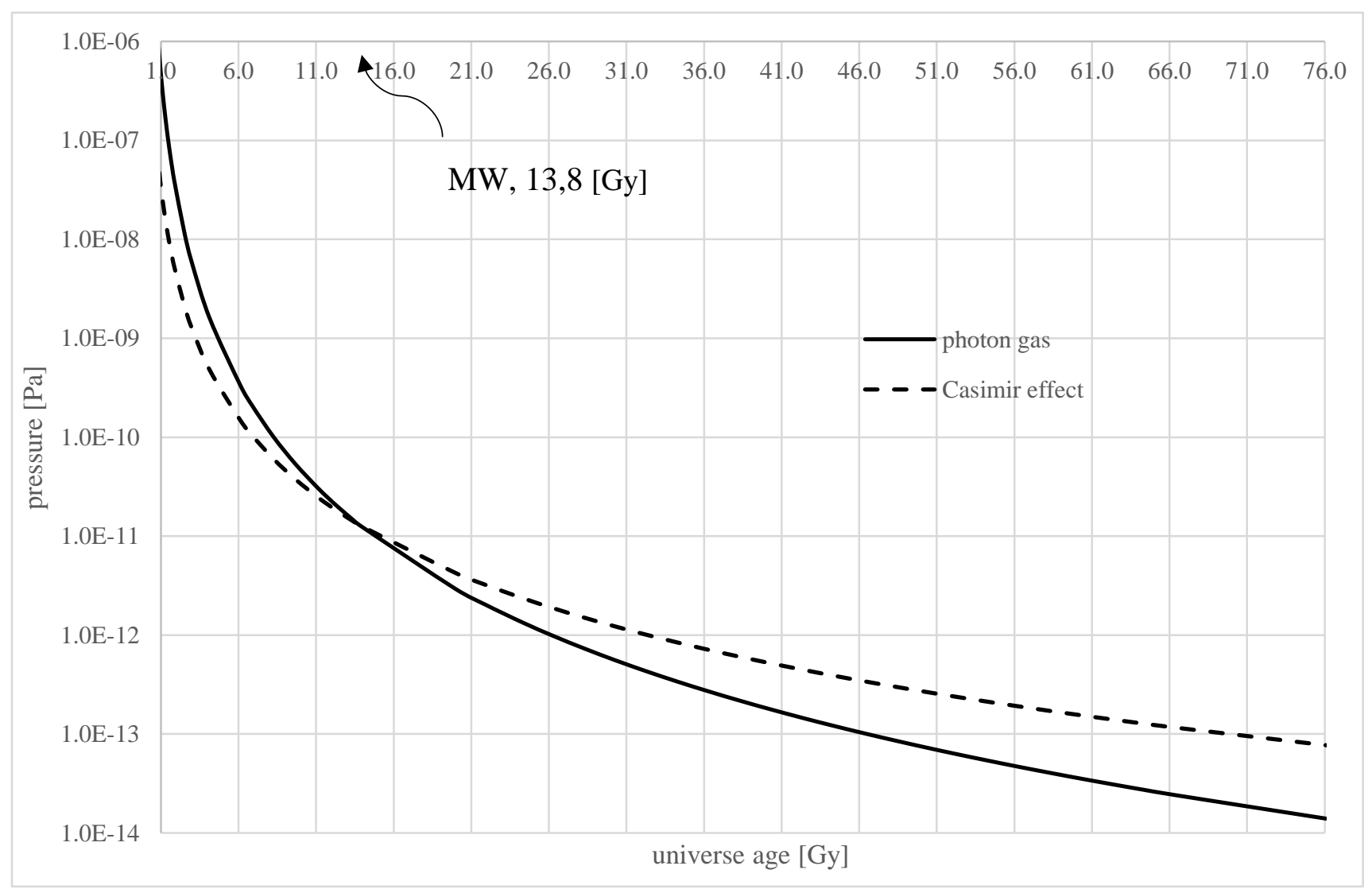

Figure 12: Photon Pg and Casimir $\mathrm{P}^{0}{ }_{\mathrm{c}}$ (energy density) from 1 [Gy] to 76.1 [Gy] 
In brief, with this model we note that photon pressure in the CMB $\left(\sim 1,291 \times 10^{-11}[\mathrm{~Pa}]\right)$ at our position, $\mathrm{t}_{0}$, provides a possible explanation for the Casimir effect, as the photons produce an amplified pressure of that value. This leads to the following question: If the Casimir effect is generated by photons in the $\mathrm{CMB}$, how is it that in laboratory experiments, in the total absence of CMB photons, when they are not physically in the presence of experimental setups, their effects are nevertheless measured by the instruments? A first part of the answer could be that the universe has stored the presence of the original photons in 'memory'. This helps us to partially understand how this effect is found in many types of experiments and phenomena (Klimchitskaya, Mohideen and Mostepanenko, 2009) : It is a fundamental characteristic of our universe, where the effects of CMB photons are stored as some sort of property of spacetime in the form of energy which we put into action and measure in diverse experimental setups with more or less pronounced amplification effects.

\section{A possible baryonic matter-free zone caused by proton and electron time lags}

This model shows that, assuming that recombination ends when the temperature drops below $\sim 3000[\mathrm{~K}]$, recombination occurred much later than the previously assumed, or $\sim 69,2[\mathrm{My}]$ rather than $\sim 380,000$ years. Now, if we calculate the redshift, $z$, at recombination, taking into account an age of $76,1[\mathrm{~Gy}]\left(2,39 \times 10^{18}[\mathrm{~s}]\right)$ for the universe, we find a redshift value that is closer to observations, or $\mathrm{Z}_{\mathrm{comb}} \sim 1100$ (Planck Collaboration, Ade, Aghanim et al., 2016):

$$
z_{c o m b}=\frac{c t_{\Omega}}{r_{c o m b}}-1=\frac{c 2,399 \times 10^{18}}{\left(\frac{3 V_{c o m b}}{4 \pi}\right)^{1 / 3}}-1 \sim \frac{7,173 \times 10^{26} \mathrm{~m}}{\left(\frac{3\left(1,172 \times 10^{72} \mathrm{~m}^{3}\right)}{4 \pi}\right)^{1 / 3}}-1=\frac{7,173 \times 10^{26} \mathrm{~m}}{6,541 \times 10^{23} \mathrm{~m}}-1 \sim 1095
$$

Or still: 


$$
z_{\text {comb }}=\frac{t_{\Omega}}{t_{c o m b}}-1=\frac{76,09 \mathrm{Ga}}{0,06919 \mathrm{Ga}}-1 \sim 1098
$$

This is a surprising result, as it matches the sequence between the temperature drop to the recombination level, around $3000[\mathrm{~K}]$, and the time period associated with recombination with the estimated age of the universe. Moreover, the redshift is calculated according to the scale factor for the universe, and not that of the MW; therefore, it applies to the entire universe rather than a onetime object within the universe. Indeed, during recombination, free photons end up on this last scattering surface, travelling in all directions, including that of expansion at the same speed as the physical boundary of the universe, $\mathrm{c}$, (we chose $\mathrm{H}=1 / \mathrm{t})$. That is why CMB photons appear as an omnipresent gas in all directions and close to us. Finally, such a late recombination time allows solving the horizon problem paradox from a standpoint of last scattering surface dimension. Indeed, the diameter of the universe at recombination was $\sim 138$ [My], making it possible to estimate the dimension of the last scattering surface with the equation for the angular dimension of a structure relative to redshift, z, and Sitter's apparent angular dimension $\Delta \theta$. For an apparent angular dimension of this last scattering surface, which covers the entire celestial half-sphere $(\Delta \theta=\pi)$, we can solve for $\mathrm{d}$ or $\mathrm{t}$ :

$$
d=c t=\frac{2 c}{H_{0}}\left[1-\frac{1}{\sqrt{1+z}}\right] \frac{\Delta \theta}{(1+z)}
$$

$t=2\left[1-\frac{1}{\sqrt{1+z}}\right] \frac{\Delta \theta}{(1+z)} t_{0}=2\left[1-\frac{1}{\sqrt{1+1100}}\right] \frac{\pi}{(1+1100)} 4,35 \times 10^{17}[s]=2,408 \times 10^{15} \mathrm{~s}=76,3[\mathrm{My}]$

Then, a smaller value than the diameter of the universe at recombination, or:

$$
d_{c o m b}=2 r_{c o m b}=2 c t_{c o m b}=138[M y]
$$


We can see that the last scattering surface is included in the universe at that time, which suggests that the inflation mechanisms may no longer be in play, at least from the standpoint of the physical dimensions of the original CMB.

A possible zone of empty matter due to the time lag during photon and electron and the electrostatic force acting before recombination, around 69,2 [My], can be estimated. Indeed, prior to the creation of baryonic matter, only photons can be observed. We begin with the calculation at the time of protons, $t_{p r}=9,939 \times 10^{-13}[\mathrm{~Gy}]$ (advent of the baryon mass). Using an expanding sphere of matter from before recombination at 69,2 [My], well before the formation of structures, and a mean value of $\bar{\beta}=0,998$, or the relativist value used at the time of proton creation, such a sphere of free protons and electrons, when entered into the expansion equation, yields:

$$
\begin{gathered}
t_{p r}^{0}=\frac{t_{\Omega}}{t_{1}} \frac{r_{1}}{c}+\frac{t_{1}}{t_{\Omega}} \bar{\beta}\left(t_{\Omega}-t_{1}\right)=\frac{2,399 \times 10^{18}}{2,181 \times 10^{15}} \frac{9,397 \times 10^{12}}{c}+\frac{2,181 \times 10^{15}}{2,399 \times 10^{18}} 0,998\left(2,399 \times 10^{18}-2,181 \times 10^{15}\right) \\
t_{p r}^{0}=2,177 \times 10^{15} \mathrm{~s}=69,0[\mathrm{My}]
\end{gathered}
$$

How can that $t_{p r}^{0}$ value be interpreted? First, that zone is not observable because it is prior to recombination (69,2 [My]). However, it closely corresponds to the typical range of a time period called recombination ( -200,000 years). Indeed, protons and neutrons appear approximately 666 days before electrons. At that time, the electrostatic repulsive force of protons is dominant and much greater than gravity $\left(10^{42}\right.$ times greater $)$. This repulsive action of protons, which pushes them towards the physical boundary of the universe, can be estimated. Indeed, assuming that the minimum energy principle applies at this time period of the universe, which is much greater than Planck time $\left(\mathrm{t}_{\mathrm{p}}=10^{31}\right)$, the electrostatic energy difference between an evenly distributed proton 
configuration in the volume at the time of electrons vs. evenly distributed protons around the perimeter, is:

$$
W_{e}^{V}-W_{e}^{S}=\frac{1}{2} \iiint_{V} \rho_{e} V^{*} d V-\frac{1}{2} \iiint_{S} \sigma V^{*} d S=\frac{3 Q^{2}}{20 \pi \varepsilon_{0} R}-\frac{Q^{2}}{8 \pi \varepsilon_{0} R}=\frac{Q^{2}}{40 \pi \varepsilon_{0} R}
$$

Where:

$\rho_{e}=\rho_{c t e}:$ the volumic density of proton charge in the R-radius sphere

$\sigma:$ the surface density of proton charge at $\mathrm{r}$ radius (at electron time)

$V^{*}:$ the electric potential

Q: the total charge of protons, $Q=n_{p r} \mathrm{q}$

Note that the minimum energy is for the proton configuration around the perimeter of the volume at electron time. The mean speed of proton motion towards the perimeter, discounting the effects of gravity force, which is much smaller than the Coulomb force, can be estimated using the proton motion equation with energy conservation and work done:

$$
W_{e}^{V}-W_{e}^{S}=\int_{0}^{R} \bar{F} \mathrm{dr}=\int_{0}^{R} \bar{m} \bar{a} \mathrm{dr}=\int_{0}^{R} \rho V \bar{a} \mathrm{dr}=\int_{0}^{R} \rho V \overline{\ddot{r}} \mathrm{dr}=\frac{Q^{2}}{40 \pi \varepsilon_{0} R}
$$

With the last two expressions and derivation, we get:

$$
\begin{gathered}
\frac{d}{d r}\left[\int_{0}^{R} \rho V \overline{\ddot{r}} \mathrm{dr}\right]=\frac{d}{d r}\left[\frac{Q^{2}}{40 \pi \varepsilon_{0} r}\right] \\
\rho V \overline{\ddot{r}}=\frac{-Q^{2}}{40 \pi \varepsilon_{0} r^{2}}
\end{gathered}
$$


Finally, for $r_{p}(t)$, which represents the average position of proton motion towards the perimeter during electron production, we get the following differential equation:

$$
\begin{gathered}
\overline{\ddot{r}_{p}}=\frac{-Q^{2}}{\rho V 40 \pi \varepsilon_{0} r^{2}}=\frac{-3 Q^{2}}{\frac{M_{t}}{V_{t}} 4 \pi 40 \pi \varepsilon_{0} r^{5}}==\frac{-3 Q^{2}}{\frac{3 M_{t}}{4 \pi R^{3}} 4 \pi 40 \pi \varepsilon_{0} r^{5}}=\frac{-Q^{2} R^{3}}{40 \pi \varepsilon_{0} M_{t} r^{5}} \sim \frac{-Q^{2} R^{3}}{40 \pi \varepsilon_{0} n_{p r} m_{p} r^{5}} \\
\overline{\ddot{r}_{p}} \sim \frac{-n_{p r}^{2} q^{2} c^{3} t_{p r}}{40 \pi \varepsilon_{0} n_{p r} m_{p} r^{5}}=\frac{-n_{p r} q^{2} c^{3} t_{p r}{ }^{3}}{40 \pi \varepsilon_{0} m_{p} r^{5}}=\frac{-5,51 \times 10^{114}}{r^{5}}=\frac{A}{r^{5}}
\end{gathered}
$$

Solving this equation for $\overline{r_{p}}(\mathrm{t})$ :

$$
\overline{r_{p}}(t) \sim\left(\frac{-9 A}{2}\right)^{1 / 6} t^{1 / 3}=1,67 \times 10^{19} t^{1 / 3}
$$

To find out if the protons reach the boundary of the sphere during the time period before the creation of electrons, the mean speed of the protons moving towards the perimeter can be estimated, $\dot{r_{p}}(t)$, relative to the speed of the boundary, with is equal to $\mathrm{c}$. If that speed is greater than $\mathrm{c}$, then the protons are travelling close to $\mathrm{c}$ and at the boundary of the universe during the time $t=t_{e l}-$ $t_{p r} \sim 666[\mathrm{~d}]$. Solving for the mean speed of protons $\left(\mathrm{t}=\mathrm{t}_{\mathrm{el}}\right)$, we get:

$$
\dot{r_{p}}(t)=\frac{1}{3}\left(\frac{-9 A}{2}\right)^{1 / 6} t^{-2 / 3}=\frac{5,59 \times 10^{18}}{t^{2 / 3}}=\frac{5,59 \times 10^{18}}{t_{e l} l^{2 / 3}}==\frac{5,59 \times 10^{18}}{\left(5,75 \times 10^{7}\right)^{2 / 3}}=3,75 \times 10^{13} \sim \mathrm{c}^{5}
$$

Indeed, the protons would be at the boundary at the time of electron production. Then, during electron production, even if the ionization energy of photons inhibits proton-electron recombination, they would be in a state of convergent acceleration, which would partly allay the absence of protons in that part of the universe. However, the high $m_{p} / m_{e}$ mass ratio means that possible lack of baryonic matter cannot be compensated and will remain permanent in a large area around the beginning. This has significant repercussions on the development and distribution of 
mass. Indeed, the protons, are at the periphery while the electrons in the volume are moving towards the protons but the neutrons stay distributed in the volume. Based on the calculations, there could be an area with a diameter of $\sim 135$ [My] and a boundary of $\sim 200,000$ years in depth at the limits of the observable horizon, with no baryonic matter except possibly neutrinos and other neutral particles. Such a possible baryonic matter-free zone could be the result of repulsive Coulomb force between protons, corresponding to the 666-day time lag or phase lag period between the creation of protons and electrons. That possible empty space of matter is not caused by gravity, as it acts on and creates areas of low mass density with very few galaxies or other structures, like the various areas of less matter space we can observe. This original less baryonic matter zone, if we could detect it, would point us towards the beginning of the universe, which would, of course, put into question the idea that there is no preferred position for the universe, or one of the foundations of the cosmological principle.

\section{Cosmological constant $\Lambda$ estimated values}

The Friedmann equation (FLRW metric) for an isotropic universe made up of matter in the presence of energy associated with the cosmological constant can be written in relation with the terms that contribute to the expansion or contraction of the universe, $\mathrm{H}$, with gravity, $\mathrm{G}$, the existence of energy other than baryonic through $\Lambda$ and the space curvature, $\mathrm{k}$, or:

$$
H^{2}=\left(\frac{\dot{a}}{a}\right)^{2}=\frac{8 \pi G \rho}{3}+\frac{\Lambda c^{2}}{3}-\frac{k c^{2}}{a^{2}}
$$

Where the scale factor is $a[-], \mathrm{k}$ is the space curvature, $\left[\mathrm{m}^{-2}\right]$ and $\rho$, the density of conventional mass $\left[\mathrm{kg} \mathrm{m}^{-3}\right]$. In this form, the equation represents the expansion of the universe expressed with the Hubble constant. In this model, we consider and assess the evolution of conventional energy 
(photon gas and mass-energy equivalence). An expression for the cosmological contant, $\Lambda$, can be obtained using the Friedmann equation. Indeed, assuming the existence of mass-energy equivalence (non-baryonic), represented by constant $\Lambda$, along with zero acceleration $(\mathrm{H}=0)$ of that mass-energy equivalence, that equation, which represents the non-baryonic residual volumic massenergy equivalence of the universe, is written as:

$$
\frac{E_{\text {conventional }}-E_{\text {mass-energy }}}{V}=\frac{E_{\Lambda}}{V}=\frac{m_{\Lambda} c^{2}}{V}=\rho_{e \Lambda} c^{2}=\left|\frac{3 k c^{4}}{a^{2}}-\frac{c^{4} \Lambda}{8 \pi G}\right|
$$

With space curvature $\mathrm{k}$ (closed if $\mathrm{k}>0$, flat if $\mathrm{k}=0$ and open if $\mathrm{k}<0$ ):

$$
\mathrm{k}=\left[\frac{a^{2} 8 \pi G \rho}{3 c^{2}}+\frac{a^{2} \Lambda}{3}\right]-\left[\frac{a^{2} H^{2}}{c^{2}}\right]
$$

The effects of each term of the equation are clearly seen. The first term is the closing effect caused by gravity, G, via mass density, $\rho$; the second is the closing effect caused by the residual massenergy equivalence (non-baryonic) via cosmological constant $\Lambda$; and the last term is the opening effect, or expansion, caused by an unknown element, but represented by the Hubble constant. Figure 13 shows that the space curvature, $\mathrm{k}$ (equation $\mathrm{k}(\mathrm{H})$ ), in relation to the other variables: $\rho, \Lambda$ and $\mathrm{H}=1 / \mathrm{t}$. The value of $\mathrm{k}$ today, time $\mathrm{t}_{0}$, is very close to zero, but slightly negative (open).

$$
\mathrm{k}\left(\mathrm{t}_{0}\right) \sim-5,6 \times 10^{-53}\left[\mathrm{~m}^{-2}\right]
$$




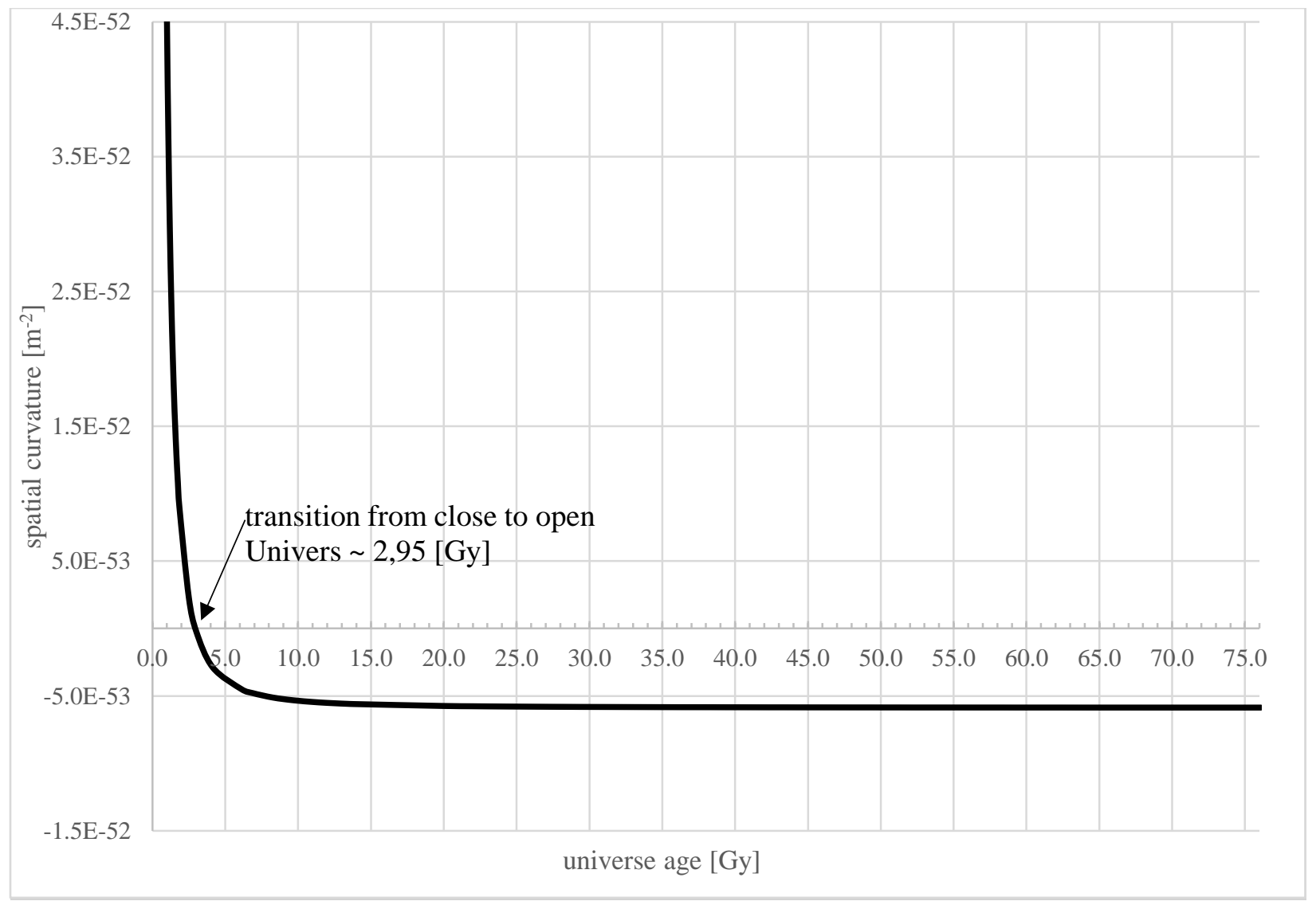

Figure 13: Spatial curvature k from 69 [My] to 76,1 [Gy]

The transition between a closed and open universe around 3 [Gy] is clear.

An oft-mentioned expression for the cosmological constant is found in the following equation (with space curvature, $\mathrm{k}$, considered to be zero), which represents the existence of a non-baryonic volumic energy density in the universe:

$$
\rho_{\Lambda e}=\rho_{\Lambda} c^{2} \sim \frac{c^{4} \Lambda}{8 \pi G}
$$

The model estimates this residual conventional energy density from the mass created at time $t$, with the equation below. Indeed, all the variables in this equation are conventional type (positive 
pressure and positive volume). There are no new-type variables which could translate the existence of a form of energy other than conventional:

$$
\rho_{\Lambda e} c^{2}=\frac{E_{\text {conventional }}-E_{\text {equivalence } m-E}}{V}=\frac{3 P V-\frac{M_{T} c^{2}}{\sqrt{1-\beta^{2}}}}{V}=\left[\frac{4 \sigma T^{4}}{c}\right]-\left[\frac{4 \sigma \frac{M_{T} c^{2}}{\sqrt{1-\beta^{2}}}}{3 c^{4} \psi k_{b} t^{3}}\right]
$$

Where $\psi=\frac{64}{3} \pi^{2} \zeta(3)\left(\frac{k_{b}}{h c}\right)^{3} \sim 8,497 \times 10^{7}\left[m^{-3} K^{-3}\right]$.

With the equation below, two dominant terms at different times are found for the expression of the cosmological constant, by virtue of the dominator, which reduces in $\mathrm{t}^{4}$ for the first term, and $\mathrm{t}^{3}$ for the second. Hence, the first dominant term for the beginning of expansion can be written as $\Lambda_{\text {rad }}$, and the second, $\Lambda_{\text {mass }}$, for the time period that comes later with the creation of the baryonic mass until today, at time $\mathrm{t}_{0}$. Moreover, the second term, which contains the mass generated over time, shows that the constant can undergo relatively quick variations:

$$
\Lambda(t)=\Lambda_{\text {rad }}-\Lambda_{\text {mass }} \sim\left[\frac{8 \pi G}{c^{4}}\right]\left[\frac{4 \sigma T^{4}}{c}\right]-\left[\frac{8 \pi G}{c^{4}}\right]\left[\frac{4 \sigma \frac{M_{T} c^{2}}{\sqrt{1-\beta^{2}}}}{3 c^{4} \psi k_{b} t^{3}}\right]=\left[\frac{32 \pi G \sigma T^{4}}{c^{5}}\right]-\left[\frac{32 \pi G \sigma \frac{M_{T} c^{2}}{\sqrt{1-\beta^{2}}}}{3 c^{8} \psi k_{b} t^{3}}\right]
$$

This predictive equation for $\Lambda$, often written $\Lambda_{\text {eff }}$, has the following characteristics: When $\mathrm{t} \rightarrow \mathrm{t}_{\mathrm{p}}$, $\Lambda=\Lambda_{\text {rad }} \rightarrow 10^{70}$, and inversely when $\mathrm{t} \rightarrow \mathrm{t}_{\Omega}, \Lambda=\Lambda_{\text {rad }}-\Lambda_{\text {masse }} \rightarrow 10^{-58}$; at $\mathrm{t}=\mathrm{t}_{0}(13,8$ [Gy]), we get the value $6,7 \times 10^{-54}\left[\mathrm{~m}^{-2}\right]$, which is in the order of magnitude of the oft-mentioned value: $<10^{-52}\left[\mathrm{~m}^{-2}\right]$, ((Padmanabhan, 2003) (Petrosian, 1974)). This value varies greatly throughout the age of the universe. Moreover, the constant is not a true constant; indeed, it varies with the age of the universe, that is to say the effects of expansion and the production of mass, or the decrease of non-massive energy in the universe. 
At the beginning, during the primitive formation of large structures like galaxies over a time period of about 0,2 to 2 [Gy], the energy is mostly in the form of radiation (over $90 \%$ of the energy is radiation), and for this period of a few [Gy], the second term, which depends on total mass, $\mathrm{M}_{\mathrm{T}}$, is far less important. Figure 14 shows the $\Lambda_{\text {mass }} / \Lambda_{\text {rad }}$ ratio.

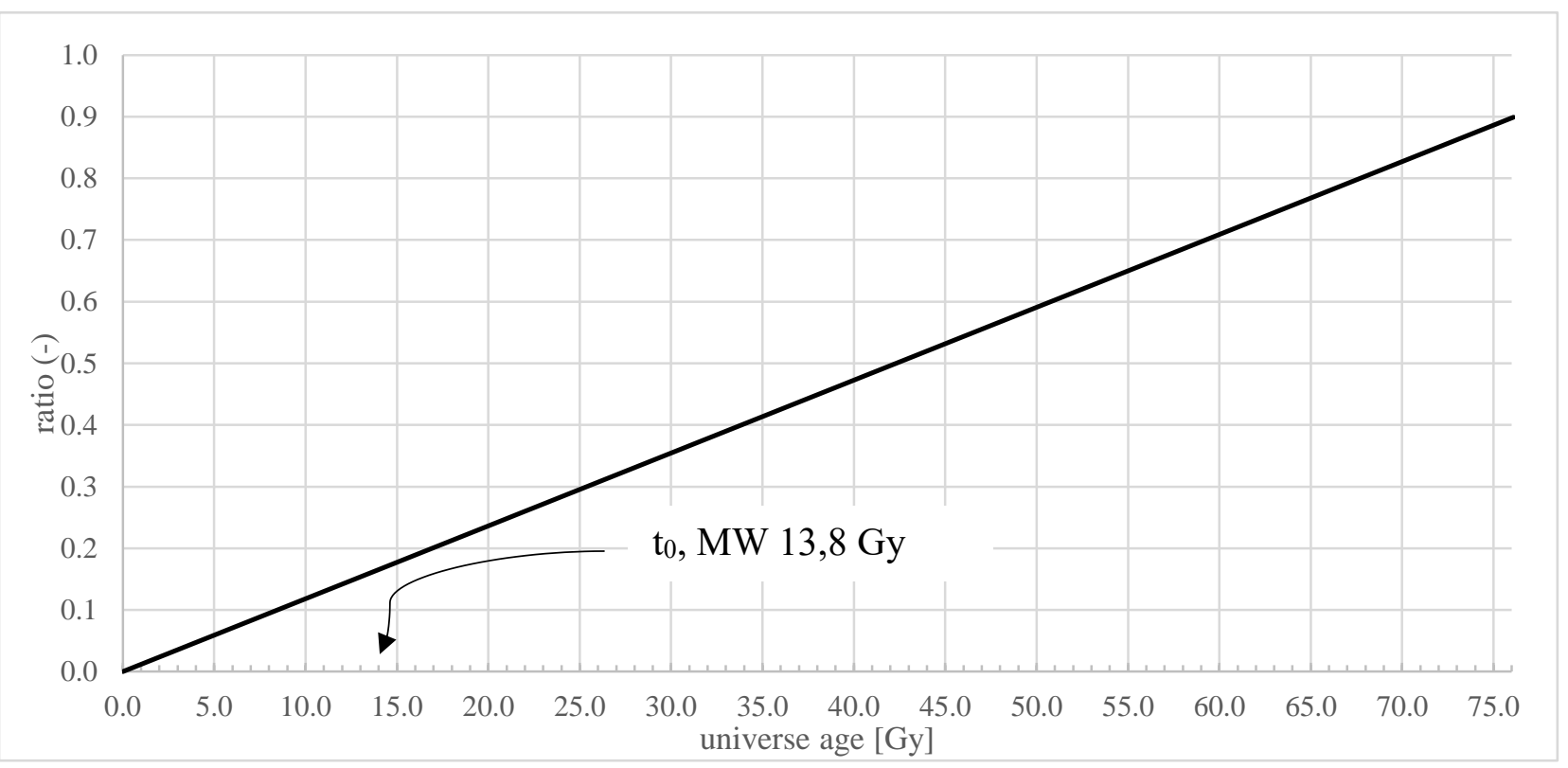

Figure 14: Ratio of $\Lambda_{\text {mass }} / \Lambda_{\text {radiation }}$

Therefore, the $\Lambda_{\text {mass }} / \Lambda_{\text {radiation }}$ ratio at our time, $\mathrm{t}_{0}$, is equal to $\sim 0,163$. It is interesting to note that the ratio obtained is in the same order of magnitude as this mentioned for baryonic matter to that of dark matter $\Omega_{\text {barionic }} / \Omega_{\text {dark }} \sim \frac{0,0457}{0,2693} \sim 0,169(\mathrm{~h}=0,7)$ (Planck Collaboration, Aghanim et al., 2018). For a universe where radiation is dominant, during the formation of large structures $(<2[\mathrm{~Gy}])$, a simplified expression can be used for the cosmological constant (Fig 15):

$$
\Lambda \sim \Lambda_{\text {rad }}=\left[\frac{32 \pi \sigma T^{4}}{P_{p}}\right]=\left[\frac{64 \pi^{6}}{15}\right]\left[\frac{k_{b}^{4} G}{h^{3} c^{7}}\right] T^{4}=1,57 \times 10^{-58} \mathrm{~T}^{4}=\left[\frac{64 \pi^{6}}{15}\right]\left[\frac{k_{b}^{4} G}{h^{3} c^{7}}\right]\left[T_{\Omega}^{4} t_{\Omega}^{4}\right] \frac{1}{(-t+b)^{4}}
$$

For $\mathrm{t}_{\Omega}=76,1[\mathrm{~Gy}]$ and $\mathrm{T}_{\Omega}=2,7[\mathrm{~K}]$ and $\mathrm{H}=1 / \mathrm{t}$ and $\mathrm{b} \sim 0$, for $\Lambda_{\text {rad }}$ and $\Lambda_{\text {mass }}$ we get: 


$$
\begin{aligned}
& \Lambda_{\text {rad }}(t>b)_{(\text {for universe age } 76,1 G y)}=\frac{k_{\Lambda}}{(-t+b)^{4}}=\frac{2,8854 \times 10^{17}}{(-t+b)^{4}}=\frac{2,8854 \times 10^{17} H^{4}}{(-1+b H)^{4}}=2,8854 \times 10^{17} H^{4}
\end{aligned}
$$

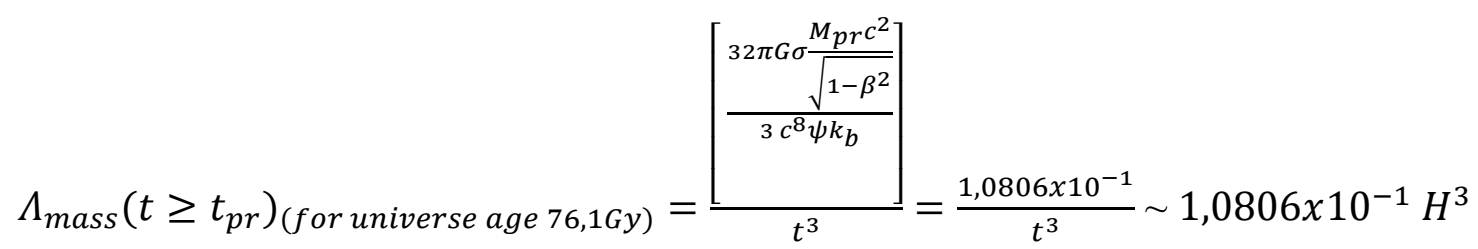

Finally, we get an approximative expression for the cosmological constant, taking only the proton mass into consideration:

$$
\begin{gathered}
\Lambda\left(b \leq \mathrm{t}<t_{p r}\right)=2,88 \times 10^{17} H^{4}\left[\mathrm{~m}^{-2}\right] \\
\Lambda\left(t \geq t_{p r}\right)=2,88 \times 10^{17} H^{4}-1,08 \times 10^{-1} H^{3}\left[m^{-2}\right]
\end{gathered}
$$

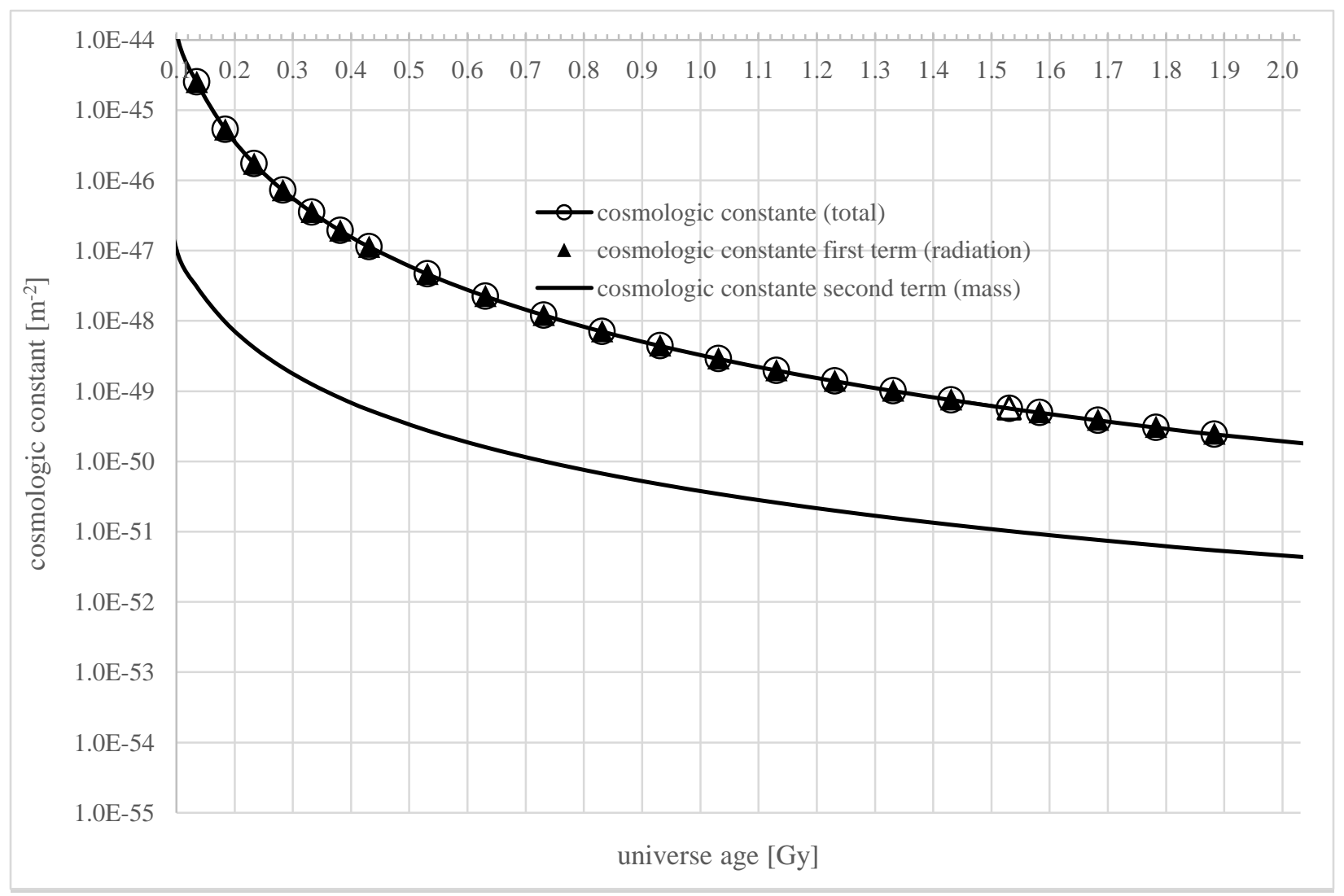

Figure 15: Cosmologic constante $\Lambda$ from 69 [My] to 2 [Gy] 
After manipulation, another expression for $\Lambda_{\text {rad }}$ is found:

$$
\Lambda_{\text {rad }}=\left[\frac{8}{r_{u}^{2}}\right]\left[\frac{P_{u}}{P_{p}}\right]=\left[\frac{8 G}{c^{5} r_{u}^{2}}\right]\left[P_{u}\right]=\left[\frac{8 H^{2}}{\dot{r}_{u}^{2}}\right]\left[\frac{P_{u}}{P_{p}}\right]
$$

The above equation contains a scale factor that varies inversely with the radius of the universe, $r_{u}^{2}$, modulated by a power ratio, or the quotient of output power of the universe, $\mathrm{P}_{\mathrm{u}}$, taken as a blackbody at $\mathrm{T}$, time $\mathrm{t}$, and Planck power $\mathrm{P}_{\mathrm{p}}$. This clearly shows that the cosmological constant diminishes relative to the squared radius and dissipated energy of the universe, leading to the great variation of the two factors combined, scale and energy. These two variations of magnitude (squared scale factor and dissipated energy) lead to the great variation of the constant. Indeed, the only variation of the energy factor $\left(\mathrm{P}_{\mathrm{u}} / \mathrm{P}_{\mathrm{p}}\right)$ leads to a variation of $\sim 10^{4}$, and that of the squared radius, to a variation of $\sim 10^{126}$. In brief, it is principally the expansion of the universe that leads to the reduction of the constant. For a static universe, the ratio of the powers is equal to 1 , and the radius remains constant, meaning that the cosmological constant would truly be a constant. The following correlation is sometimes reported:

$$
D_{u}=2 r_{u} \sim \frac{1}{\sqrt{\Lambda}}
$$

In this model the expression is:

$$
D_{u}=2 r_{u}=\frac{\sqrt{\frac{32 P u}{P p}}}{\sqrt{\Lambda}}
$$

For $\mathrm{t}=13,8[\mathrm{~Gy}]$, the constant of the numerator is: 


$$
\sqrt{\frac{32 P_{u}}{P_{p}}}=\sqrt{\frac{32\left(7,66 \times 10^{50}\right)}{3,629 \times 10^{52}}} \sim \sqrt{0,54}=0,74
$$

Another value for $\Lambda$ is suggested by Carmeli et al. (2001):

$$
\Lambda=\frac{3}{c^{2} t^{2}}=2,2642 \times 10^{-52}\left[m^{-2}\right]
$$

Where $\tau=12,16$ [Gy]. In this model, we get the following form:

$$
\Lambda=\frac{\frac{8 G}{c^{5}} P u}{c^{2} t^{2}}=\frac{0,137}{c^{2} t^{2}}=9,9 \times 10^{-54}\left[\mathrm{~m}^{-2}\right]
$$

If different Planck quantities are used, the following expression can be used for the constant:

$$
\Lambda_{\text {rad }}=8\left[\frac{P_{u}}{r_{u}^{2}}\right]\left[\frac{t_{p}^{3}}{m_{p} l_{p}^{2}}\right]=8\left[\frac{P_{u}}{r_{u}^{2}}\right]\left[\frac{1}{c F_{p}}\right]
$$

Also, this expression is for the beginning when $\mathrm{t} \rightarrow \mathrm{t}_{\mathrm{p}}$ :

$$
\Lambda\left(t_{P}\right)=\frac{32 \pi G \sigma T_{p}^{4}}{c^{5}}=\frac{16 \pi^{4}}{15} \frac{c^{3}}{G h}=\frac{\frac{8 \pi^{3}}{15}}{l_{p}^{2}}=6,33 \times 10^{70}\left[\mathrm{~m}^{-2}\right]
$$

Figures 16 and 17 show the graph for $\Lambda$. For the entire duration of the simulation, or 76,1 [Gy], the cosmological constant varies by a factor of $\sim 10^{128}$; or by $10^{70}$ at $\mathrm{t} \rightarrow \mathrm{t}_{\mathrm{p}}$ until $10^{-58}$ for $\mathrm{t}=76,1$ [Gy]. 


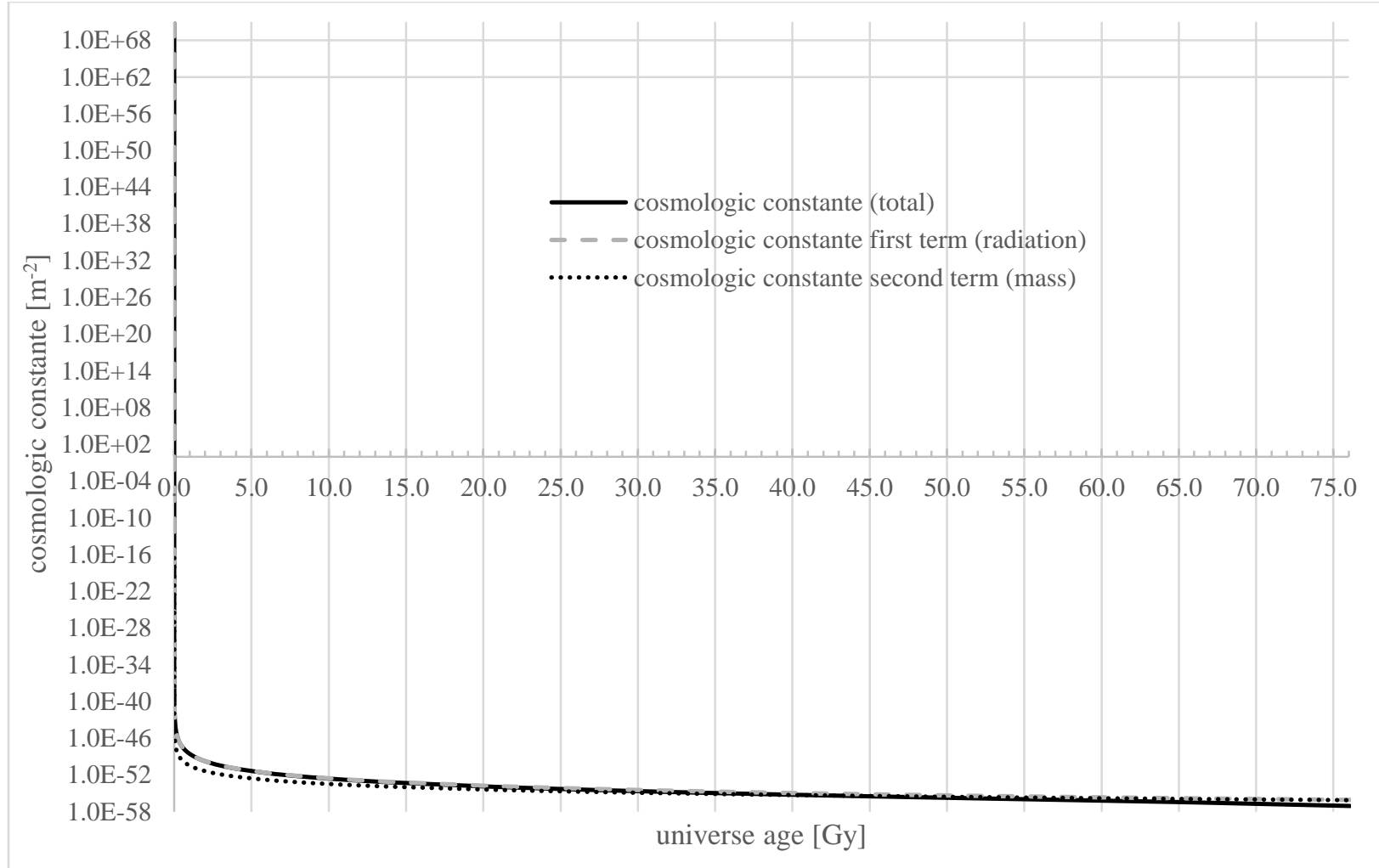

Figure 16: Cosmologic constant $\Lambda=\Lambda_{\text {rad }}+\Lambda_{\text {mass }}$ from 1 tp to 76,1 [Gy]

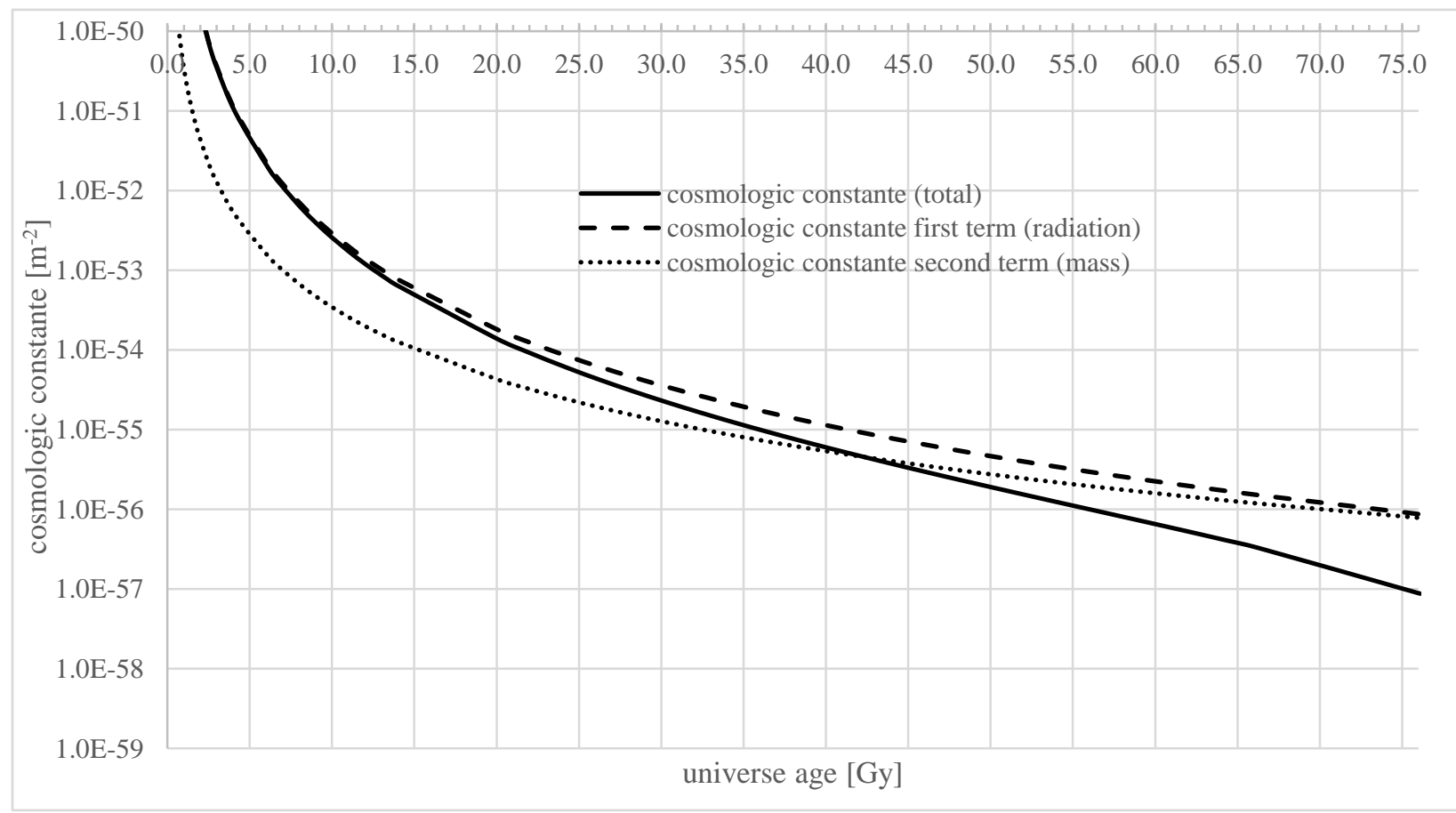

Figure 17: Cosmologic constante $\Lambda=\Lambda_{\mathrm{rad}}+\Lambda_{\text {mass }}$ from 1 tp to 76,1 [Gy] 
In brief, those expressions for space curvature and energy density (non-baryonic) can be obtained by substituting the cosmological constant equation:

$$
k(H)=a^{2}\left[\frac{k_{\Lambda}}{3(-1+b H)^{4}} H^{4}+G M_{t} \frac{\left(2-\frac{\pi^{4}}{45 \zeta 3)}\right)}{c^{5}} H^{3}-\frac{1}{c^{2}} H^{2}\right] \sim a^{2}\left[9,61 \times 10^{16} H^{4}+3,98 \times 10^{-3} H^{3}-1,11 \times 10^{-17} H^{2}\right]
$$

Where $t \geq t_{p r}$

The space curvature equation yields $\mathrm{k}=0$ for $\mathrm{t}=2,95[\mathrm{~Gy}]$, or the transition from closed to open universe. This closely corresponds with the value found for deceleration transition, q, around 2 [Gy] (Fig. 8). That these two values are relatively close is promising in terms of model constancy.

As concerns energy density, we find two distinct contributions: one associated with radiation and the other, with mass (for $b \sim 0$, valid for $t>10^{-13}[\mathrm{~s}]$ ):

$$
\rho_{\Lambda e}(H)=\rho_{\Lambda e}^{r a d}+\rho_{\Lambda e}^{m a s s}=\frac{c^{4} k_{\Lambda}}{8 \pi G(-1+b H)^{4}} H^{4}-\frac{\pi^{3} M_{t}}{120 \zeta(3) c} H^{3} \sim 1,38 \times 10^{60} H^{4}-4,21 \times 10^{41} H^{3}
$$

Figures 18 and 19 show energy density in association with the cosmological constant relative to the age of the universe. For Planck time $t_{p}$, we get an energy density of $\sim 10^{113}\left[\mathrm{~J} \mathrm{~m}^{-3}\right]$, while for $\mathrm{t}_{0}$ $(13,8[\mathrm{~Gy}])$ that number drops to $\sim 10^{-11}\left[\mathrm{~J} \mathrm{~m}^{-3}\right]$; a reduction factor of $\sim 10^{124}$ !

The expression for energy density at $t_{p}$ can be written as:

$$
\rho_{\Lambda e}\left(t_{p}\right)=\frac{\pi^{3}}{15} \frac{c^{7}}{G^{2} h}=\frac{\pi^{2}}{15} P_{p r e s} \sim 3 x 10^{113}\left[\mathrm{~J} \mathrm{~m}^{-3}\right]
$$

After a few algebraic manipulations, the following expression is obtained, yielding the energy density variation from the beginning, $\mathrm{t}_{\mathrm{p}}$, until today, $\mathrm{t}_{0}(13,8[\mathrm{~Gy}])$. 


$$
\begin{gathered}
\frac{\rho_{\Lambda e}\left(t_{0}\right)}{\rho_{\Lambda e}\left(t_{p}\right)}=\left(\frac{T_{\Omega}}{T_{P}}\right)^{4}\left(\frac{\zeta(3)}{\zeta(4)}\right)^{4} \frac{1}{\left(5+W_{0}\left(-5 e^{-5}\right)\right)^{4}} \\
\frac{\rho_{\Lambda e}\left(t_{0}\right)}{\rho_{\Lambda e}\left(t_{p}\right)} \sim 1,081 \times 10^{-3}\left(\frac{T_{\Omega}}{T_{P}}\right)^{4} \sim\left(\frac{1,202}{\frac{\pi^{4}}{90}}\right)^{4} \frac{1}{(4,965)^{4}}\left(\frac{2,728}{1,41 \times 10^{32}}\right)^{4} \sim 1,2 \times 10^{-124}
\end{gathered}
$$

In short, as concerns energy density variation in the universe, we find a ratio to the power of four between temperature variation and Planck temperature variation, with a multiplication factor.

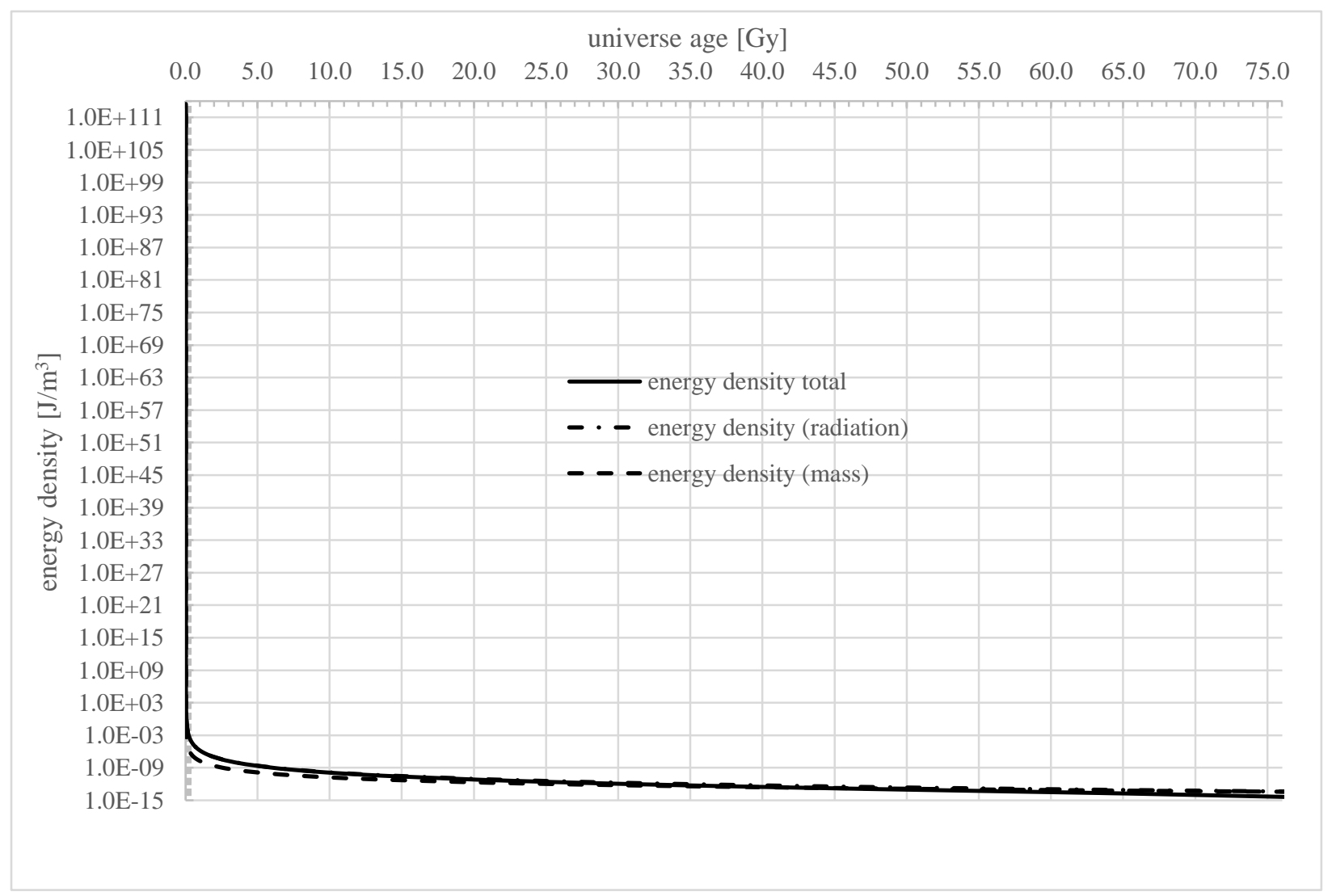

Figure 18: Energy density $\rho_{\Lambda \mathrm{e}}$ of cosmologic constant $\Lambda$ from 1 tp to 76,1 [Gy] 


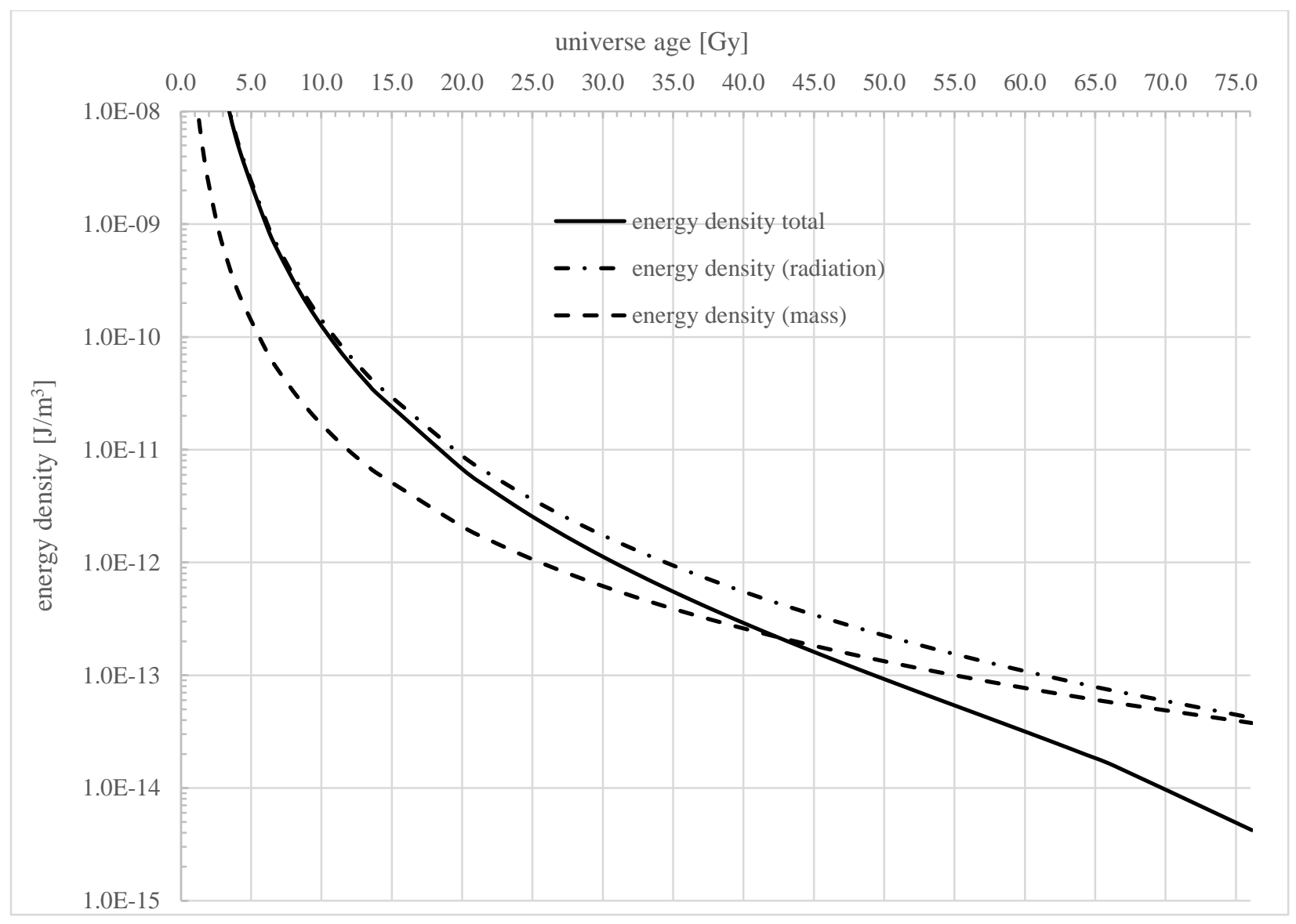

Figure 19: Energy density $\rho_{\Lambda \mathrm{e}}$ of cosmologic constant $\Lambda$ from 1 tp to $76,1[\mathrm{~Gy}]$

Finally, the Friedmann equation can be written according to the different terms of the equation in the form of an equivalent volumic mass. This highlights the relative contribution of the terms:

$$
H^{2}=\left(\frac{\dot{a}}{a}\right)^{2}=\frac{8 \pi G}{3}\left(\rho_{m}+\rho_{\Lambda}+\rho_{k}\right)
$$

The expressions of equivalent volumic mass in the equation are as follows:

$$
\rho_{m}=\frac{M_{t}}{V}
$$




$$
\begin{gathered}
\rho_{\Lambda}=\rho_{r}=\frac{E_{\gamma}-E_{M}}{c^{2} V}=\frac{2,7 N k_{b} T-M_{t} c^{2}}{c^{2} V}=\frac{3 P V-M_{t} c^{2}}{c^{2} V}=\frac{3 P}{c^{2}}-\rho_{m}=\frac{4 \sigma T^{4}}{c^{3}}-\rho_{m}=\frac{\Lambda c^{2}}{8 \pi G} \\
\rho_{k}=\frac{-3 k c^{2}}{8 \pi G a^{2}}
\end{gathered}
$$

We can see that the volumic mass associated with the cosmological constant, $\Lambda$, is equivalent to that of photon gas minus the baryonic mass. Therefore, the cosmological constant reveals the existence of radiation energy. As concerns space curvature, we get a value that can turn negative according to the value of the curve (closed universe). This is important data because it is the only term that can become negative and act in opposition to gravity and mass-energy equivalence. If we express volumic masses based on the critical value corresponding to $\Lambda=k=0$, or a flat universe whose only energy comes from mass, we get:

$$
\rho_{c r i}=\frac{3 H^{2}}{8 \pi G}
$$

With

$$
\Omega_{m}=\frac{\rho_{m}}{\rho_{c r i}}, \Omega_{\Lambda}=\frac{\rho_{\Lambda}}{\rho_{c r i}}, \Omega_{k}=\frac{\rho_{k}}{\rho_{c r i}}
$$

In the Friedmann equation:

$$
H^{2}=\left(\frac{\dot{a}}{a}\right)^{2}=\frac{8 \pi G}{3}\left(\Omega_{m}+\Omega_{\Lambda}+\Omega_{k}\right) \rho_{c r i}
$$

Figures 16 and 17 show the values for $\rho_{i}$ and $\Omega_{i}$ calculated according to the age of the universe. 


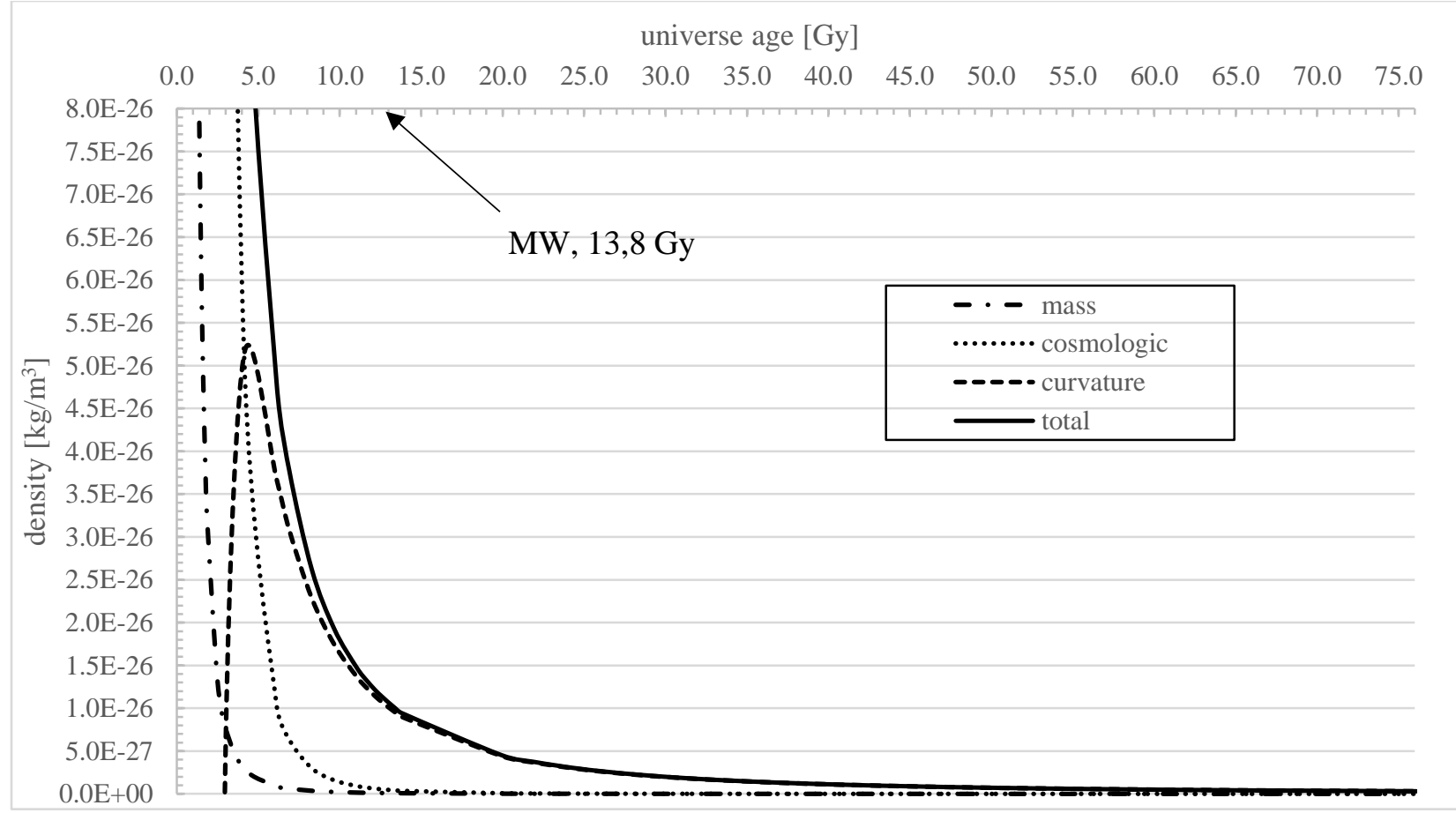

Figure 20: Equivalent densities $\rho$ of Friedmann equation terms from $1 t_{p}$ to 76,1 [Gy]

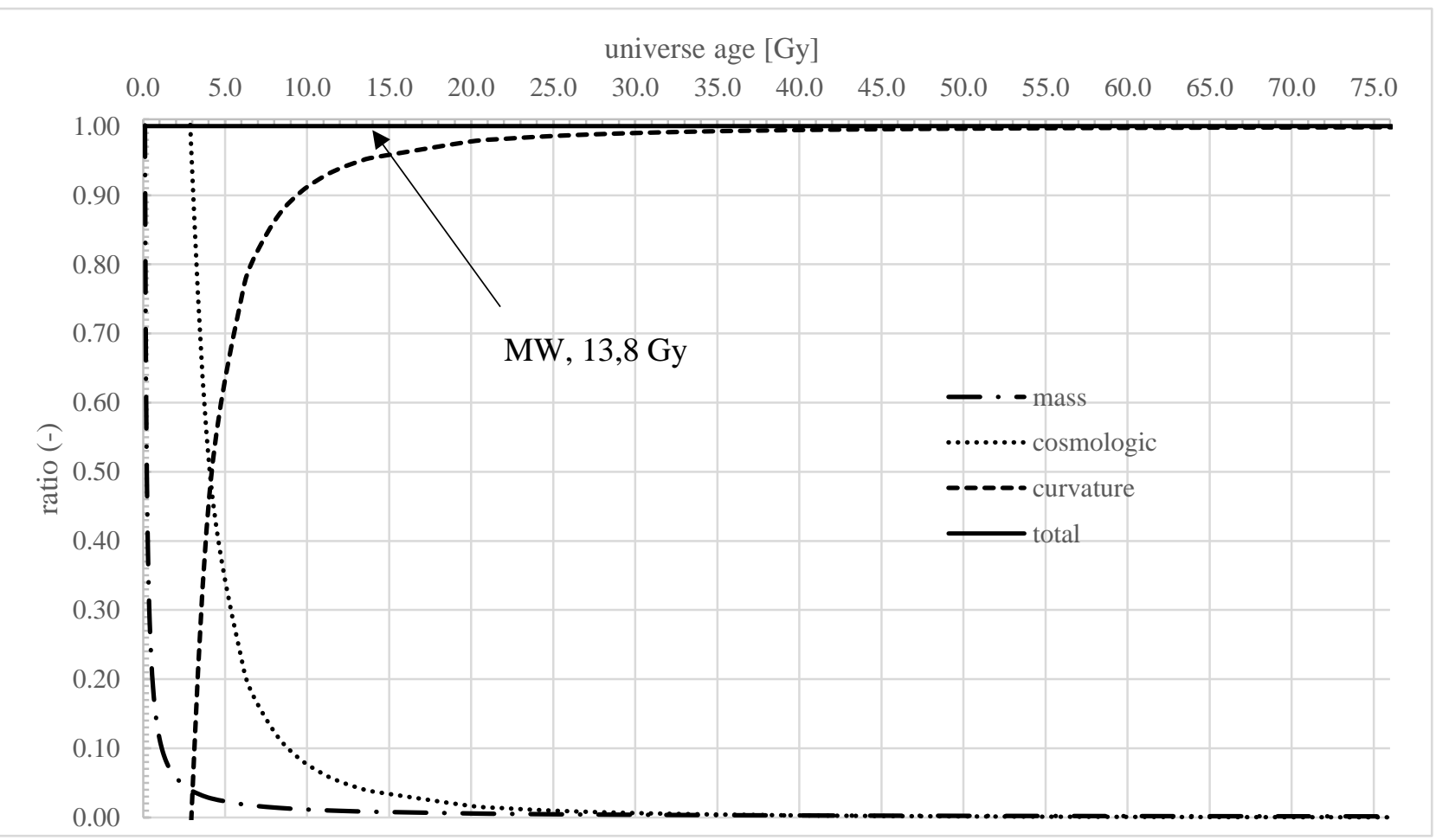

Figure 21: Ratio of densities $\Omega$ of Friedmann equation from $1 t_{p}$ to 76,1 [Gy] 
Figure 20 shows the equivalent densities. Here, the contribution of curvature is negative for an age below 2,9 [Gy], a closed universe, as already discussed with the q curve (deceleration). Then, that value of curvature increases rapidly to about $4[\mathrm{~Gy}]$. Thereafter, all values decrease in monotonic fashion and at different rates. Note that the total value is very close to the critical value, but always smaller.

Figure 21 shows the values of associated contributions as they relate to critical density. We can see that curvature, $\mathrm{k}$, is the key factor that can explain sustained expansion of the universe. We know that the contribution of mass, along with the cosmological constant, are based on conventional energy (mass-energy, radiation). In the case of space curvature, $\mathrm{k}$, that form of energy cannot be so easily explained.

\section{The energy form of the Friedmann equation}

To determine the type of energy behind the expansion of the universe, the Friedmann equation can be expressed in terms of energy. Indeed, if all the terms of the equation are multiplied by $c^{5} G^{-1} H^{-3}$, we get:

$$
\begin{gathered}
\left(\frac{c^{5}}{G H^{3}}\right) H^{2}=\left(\frac{c^{5}}{G H^{3}}\right) \frac{8 \pi G \rho_{m}}{3}+\left(\frac{c^{5}}{G H^{3}}\right) \frac{\Lambda c^{2}}{3}-\left(\frac{c^{5}}{G H^{3}}\right) \frac{k c^{2}}{a^{2}} \\
\frac{c^{5}}{G H}=\frac{c^{5}}{H^{3}} \frac{8 \pi \rho_{m}}{3}+\frac{c^{7}}{G H^{3}} \frac{\Lambda}{3}-\frac{c^{7}}{G H^{3}} \frac{k}{a^{2}}
\end{gathered}
$$

Let us express density with total mass and radius using the Hubble-Lemaitre law for the boundary $(c=H r)$, as: 


$$
\rho_{m}=\frac{M}{V}=\frac{M}{\frac{4 \pi}{3} r^{3}}=\frac{3 M}{4 \pi r^{3}}=\frac{3 M H^{3}}{4 \pi c^{3}}
$$

Finally, we get an expression of the Friedmann equation in the form of energy:

$$
\begin{gathered}
\frac{c^{5}}{G H}=2 M c^{2}+\frac{c^{7}}{G H^{3}} \frac{\Lambda}{3}-\frac{c^{7}}{G H^{3}} \frac{k}{a^{2}} \\
E_{\text {Planck }}=E_{\text {mass }}+E_{\text {radiation }}+E_{\text {curvature }}
\end{gathered}
$$

Let us express the energy associated with curvature as:

$$
\begin{gathered}
E_{\text {curvature }}=E_{\text {Planck }}-E_{\text {mass }}-E_{\text {radiation }} \\
E_{\text {curvature }}=\frac{P_{\text {Planck }}}{H}-2 M c^{2}-\frac{c^{7}}{G H^{3}} \frac{\Lambda}{3} \\
E_{\text {curvature }} \sim \frac{P_{\text {Planck }}}{H}-2 M c^{2}-\frac{c^{7}}{G} \frac{k_{\Lambda} H}{3}
\end{gathered}
$$

Where: $k_{\Lambda}=2,88 \times 10^{17}\left[s^{4} \mathrm{~m}^{-2}\right], M \sim 7,53 \times 10^{50}[\mathrm{~kg}], P_{\text {Planck }}=3,629 \times 10^{59}[\mathrm{~W}]$

A positive energy result represents an open universe, while a negative result means a closed universe. In the above equation, note that both positive and negative results are possible according to the values of the terms. The first term, open, is Planck power multiplied by cosmic time. The second term, closed, is a constant of total energy associated with mass (50\% energy, 50\% kinetic, $\bar{\beta}=\sqrt{3 / 4}$ ), and the third term, closed, is the energy associated with radiation (via $\Lambda$ ), which decreases with the increase in cosmic time. The transition from a closed universe to an open one is for $\mathrm{E}_{\text {curvature }}=0$. We get the following positive root:

$$
H=1,054 \times 10^{-17}\left[s^{-1}\right]
$$




$$
t=\frac{1}{H}=3,00[\mathrm{~Gy}](\mathrm{z} \sim 3,6)
$$

In the above equation, if the mass is increased by a factor of 10 or 50 the transition from close to open is delayed by $590[\mathrm{My}]$ or 4,1 [Gy] (at 3,59 [Gy] or 7,1 [Gy]). We see the impact of the mass on the transition.

In short, with the Friedmann equation and the assumptions of this model, we find that energy of unknown origin is acting on the expansion of the universe through an enormous power that is equal to Planck power $P_{P}$ multiplied by cosmic time. That expansion energy is not directly expressed in a model variable. Moreover, it is positive via Planck power, which represents conventional energy acting in opposition to gravity and cosmological gravity force. The expansion power is not associated to mass (baryonic) or radiation (photonic via $\Lambda$ ). This unknown energy of expansion is possibly contained in a potential form available at the frontier of the universe that acts by an expansion effect of space in the manner of a stretching of space. This Planck power $P_{P}$ can be expressed by the Planck force $F_{P}$ multiplied by c. In this model, we consider that the frontier of the universe moves at speed c (constant). It is seen that the idea of an internal or external force (multiverse) of the magnitude of Planck force acts at the boundary to stretch the space at speed c.

\section{Some comparison with some data from the $\Lambda \mathrm{CDM}$ model}

The table 2 below shows some of the major differences between this model and the $\Lambda$ CDM model (Planck Collaboration, Aghanim et al., 2018) The numbers are averages over a time period ranging from $\mathrm{z}=0$ to $\sim \mathrm{Z}_{\mathrm{re}}(\sim 7,70)$, or $\sim 1,5[\mathrm{~Gy}]$ to $13,8[\mathrm{~Gy}]$. Variations in values were left out for easier comparison. Indeed, Planck measurements are from different times in the past of the universe, thus confirming that they are, at least partly, time-related averages. The table shows three main 
differences: First, the estimated age of the universe is greater. The MW is situated at cosmic time 13,8 [Gy]; second, the baryonic mass is 11 times smaller; and third, dark energy associated with $\Lambda$ is in fact radiation energy, which is very large at the beginning. Moreover, if the total energy associated with dark matter in added up in the $\Lambda$ CDM model, dark energy and radiation $\left(\Omega_{c d m}+\Omega_{\Lambda}+\Omega_{r}\right)$, we get $\sim 0,950$, which is quite similar to the radiation value, $\Omega_{\Lambda}$, of the model $(0,968)$.

Finally, at the beginning, the energy associated with space curvature, $\mathrm{k}$, is relatively small compared to radiation. That energy is of unknown origin and possibly acting at the boundary. As concerns the curvature of space, $\mathrm{k}$, the energy source is not identified. However, in this version of the model, that energy form does not behave like mass-energy equivalence, as is the case with the cosmological constant.

Table 2: Some comparison between this model $\mathrm{A} \Lambda \Omega$ and $\Lambda \mathrm{CDM}$ model

Description

Age of the universe (cosmic)

Total density parameter

Hubble constant Symbol

$\mathrm{t} \Omega[G y]$

$\Lambda C D M$

13,799 (obs.)

46

$\Omega_{\text {tot }}$

1

$H_{0} \quad 67,4 \pm 0,5$

$\left[\mathrm{km} \mathrm{s} s^{-1} M p c^{-1}\right]$

\begin{tabular}{ccccc}
\hline Baryonic density & $\Omega_{b}$ & 0,0486 & 0,03045 & Less baryonic matter \\
\hline Dark matter density & $\Omega_{c d m}$ & 0,2664 & 0 & $\begin{array}{c}\text { Dark matter is not a parameter } \\
\text { of the model }\end{array}$ \\
\hline Matter density & $\Omega_{m}$ & 0,315 & 0,03045 & Baryonic matter only \\
\hline Dark energy density & $\Omega_{\Lambda}$ & 0,685 & 0 & $\begin{array}{c}\text { Mass and/or photon energy } \\
\text { only, except for curvature } \mathrm{k}\end{array}$ \\
\hline Radiation energy density & $\Omega_{r}$ or $\Omega_{\Lambda}$ & $\Omega_{r}=10^{-5}$ & $\Omega_{\Lambda}=0,96893$ & $\begin{array}{l}\text { The cosmological constant } \\
\text { represents radiation energy }\end{array}$ \\
\hline
\end{tabular}

13,799 (obs.)

76,1

[Gy]

\section{Comments}




\begin{tabular}{lcccc} 
Curvature energy density & $\Omega_{k}$ & $+0,001 \pm 0,002$ & $+0,00062 \quad \begin{array}{c}\text { Curvature energy is of unknown } \\
\text { origin }\end{array}$ \\
Cosmic neutrino mass & $\mathrm{m}_{v}[\mathrm{eV}]$ & $\sum \mathrm{m}_{v} \leq 0,12 \quad \leq 48 \times 10^{3}[\mathrm{eV}]$ & $\begin{array}{l}\text { The cosmic neutrino is } \\
\text { estimated with the muonic } \\
\text { neutrino with } \beta \text { SN1987A }\end{array}$ \\
\hline
\end{tabular}

\section{Cosmological gravity force, $F_{\Lambda}$}

For the time period when radiation was dominant, a central force associated with $\Lambda_{\text {rad }}$ can be determined using mass-energy equivalence. Indeed, we know the value for $\Lambda_{\text {rad }}$ via the evolution of energy in the universe. Let us assume an element with mass $m$ in rotation according to a Kepler model in a central gravity field of mass $\mathrm{M}$. Another attractive force is a work around mass $\mathrm{m}$, this time associated with the non-baryonic energy density, which acts through mass-energy equivalence of the interior sphere whose boundary is determined by the rotation radius, $r$, of mass $\mathrm{m}$. That central force has been suggested by several authors, including Martin (2012). However, after mathematical elaboration, they note that the force is repulsive, and not attractive. This can be explained through mathematical calculations using the cosmological constant, which predicts a repulsive rather than attractive effect when placed on the left side of the general relativity equation.

In this model, we consider that the force is attractive simply through mass-energy equivalence, meaning that a positive energy mass is associated with a positive energy, such as the energy of photons associated with constant $\Lambda$, and that energy mass exerts an attractive force on surrounding masses the same way the inertial mass (baryonic) does. What's more, the notion of mass energy (or electromagnetic) was addressed initially by Langevin (1913), a contemporary of Einstein.

$$
m_{\text {energetic }}=m_{\text {inertial }}=m_{\Lambda}=\frac{E_{\Lambda}}{c^{2}}=\rho_{\Lambda} V=\frac{c^{2} \Lambda r^{3}}{6 G}
$$


We can see that the mass energy associated with the cosmological constant (photon gas) depends on a zone demarcated by the assumed radius, $r$. The full action of this force is unknown, but it is gravitational, meaning that this cosmological gravity force acts together with conventional gravity and that other such couplings are possible. This can partially explain the issues with the cosmological constant, $\Lambda$. In fact, that gravity force can be put into action in the general relativity equation through the existence of the cosmological constant, as put forth by Einstein but for a different reason than the static universe he proposed. Indeed, the cosmological constant was later added by Einstein as an opposing force to gravity. Therefore, when the term $\Lambda g_{\mu \nu}$ is moved to the right-hand side, the side of the energy-momentum tensor, we get a repulsive effect associated with $\Lambda:$

$$
R_{\mu \nu}-\frac{1}{2} R g_{\mu \nu}+\Lambda g_{\mu \nu}=\frac{8 \pi G}{c^{4}} T_{\mu \nu}
$$

With the signature of the metric tensor $(+,-,-,-)$, the energy-momentum tensor can be expressd as:

$$
T_{\mu \nu}^{\text {total }}=T_{\mu \nu}^{\text {baryonic }}-\rho_{\Lambda e} g_{\mu \nu}
$$

In this case, the resulting force is repulsive, as Einstein wanted. However, it is also possible to make the effects of that energy appear directly in the energy-momentum tensor as a source of additional mass energy through the mass-energy principle, as:

$$
\begin{gathered}
T_{\mu \nu}^{\text {total }}=T_{\mu \nu}^{\text {baryonic }}+T_{\mu \nu}^{\text {mass energy }} \\
T_{\mu \nu}^{\text {total }}=T_{\mu \nu}^{\text {baryonic }}+\frac{E_{\Lambda}}{V} g_{\mu \nu} \\
T_{\mu \nu}^{\text {total }}=T_{\mu \nu}^{\text {baryonic }}+\rho_{\Lambda e} g_{\mu \nu}=\rho_{m} c^{2}+\frac{c^{4} \Lambda}{8 \pi G} g_{\mu \nu}
\end{gathered}
$$


Hence, the energy density component of the tensor, $T^{00}$, is entirely positive:

$$
T^{00}=\rho_{m} c^{2}+\frac{c^{4} \Lambda}{8 \pi G}=\rho_{m+\Lambda} c^{2}
$$

The solution for the spherical geometry is found in the Newton equation for low velocities:

$$
\nabla^{2} \Phi=4 \pi G \rho_{m+\Lambda}=4 \pi G\left(\rho_{m}+\frac{c^{2} \Lambda}{8 \pi G}\right)=4 \pi G \rho_{m}+\frac{c^{2} \Lambda}{2}
$$

The potential being:

$$
\Phi=-\frac{G m}{r}+\frac{c^{2} \Lambda r^{2}}{12}
$$

A potential in $r^{2}$ is said harmonic and the equation of the trajectory of a mass $m^{\prime}$ in harmonic potential is a closed curve like that Newtonian in $\mathrm{r}^{-1}$ (Bertrand's problem). The acceleration of a mass $m^{\prime}$ in this field is expressed as the gradient of potential $\Phi$ :

$$
\begin{gathered}
\boldsymbol{a}=-\boldsymbol{\nabla} \Phi \boldsymbol{e}_{\boldsymbol{r}}=-\frac{\partial \Phi}{\partial r} \boldsymbol{e}_{\boldsymbol{r}}=-\frac{\partial\left(-\frac{G m}{r}+\frac{c^{2} \Lambda r^{2}}{12}\right)}{\partial r} \boldsymbol{e}_{\boldsymbol{r}} \\
\boldsymbol{a}=-\frac{G m}{r^{2}} \boldsymbol{e}_{\boldsymbol{r}}-\frac{\Lambda}{6} c^{2} r \boldsymbol{e}_{\boldsymbol{r}}
\end{gathered}
$$

We can see that, at this time, solving the equation predicts an attractive force associated with constant $\Lambda$ and of the same type as the baryonic mass. The $r$ term can be related to the Hooke ellipse. Moreover, it is surprising to note here that at the beginning of the formation of the structures of the universe the two forces in $\mathrm{k} \mathrm{r}^{-2}$ and $\mathrm{k} \mathrm{r}$ acted simultaneously which, certainly would be likely to reconcile, if it were possible Newton and Hooke. It would make sense to call the potential found $\mathrm{NcH}$ for Newton-cosmological-Hooke. Finally, in a detailed form, the $\mathrm{NcH}$ potential is expressed as: 


$$
\Phi(\mathrm{m}, \mathrm{r}, \mathrm{H})=-\frac{G m}{r}+\frac{c^{2} k_{\Lambda}}{12} H^{4} r^{2}
$$

Then, solving the equation for low velocities (Newton) includes two mass contributors, baryonic and energy (cosmological). At this time, we can see that expansion of the universe is not caused by dark energy associated with $\Lambda$, but by another effect seen earlier, the energy associated with curvature, $\mathrm{k}$. If that choice had been made, the force derived by Martin (2012) would be attractive.

Finally, based on this approach, we can see that the cosmological constant must be included in Einstein's equation because it represents non-baryonic energy in the universe, but the sign for the term $\rho_{\Lambda e} g_{\mu \nu}$ on the right-hand side of the equation must be positive, which provides a possible explanation for the additional attractive gravity effects associated with the positive energy of constant $\Lambda$. At this time, expansion of the universe can be attributed to energy associated with curvature, $\mathrm{k}$, as stated earlier. A similar potential has been proposed by (Farnes, 2018) but the sign of the term in $r^{2}$ is negative which forces to consider the existence of a negative mass to produce a positive attraction force. The existence of the negative mass, although possible in theory, has not been observed until now.

Therefore, assuming this notion of mass-energy, and according to Newton's law of attraction for that mass, $\mathrm{m}_{\Lambda}$, the central attractive force associated with the mass-energy equivalence can be written as:

$$
\left|F_{\Lambda}\right|=\frac{G m_{\Lambda} m}{r^{2}}=\frac{G\left(\rho_{\Lambda} V\right) m}{r^{2}}=\frac{G\left(\rho_{\Lambda} 4 \pi r^{3}\right) m}{3 r^{2}}=\frac{4 \pi G\left(\rho_{\Lambda}\right) r m}{3}=\frac{4 \pi G}{3}\left(\frac{c^{2} \Lambda}{8 \pi G}\right) r m=\frac{\Lambda}{6} c^{2} m r
$$

The force can be expressed in relation to the age of the universe:

$$
\mathrm{F}_{\Lambda}=\frac{\Lambda}{6} c^{2} r m=G\left(\frac{16 \pi \sigma T_{\Omega}^{4} t_{\Omega}^{4}}{3 c^{3}}\right) \frac{r m}{t^{4}}=G\left(\frac{16 \pi \sigma T_{\Omega}^{4} t_{\Omega}^{4}}{3 c^{3}}\right) m r H^{4}
$$


For $\mathrm{T}_{\Omega}=2,7[\mathrm{~K}], \mathrm{t}_{\Omega}=76,1[\mathrm{~Gy}]$, and $\mathrm{t}=\mathrm{t}_{0}=13,8[\mathrm{~Gy}]$, we get;

$$
\mathrm{F}_{\Lambda}=4,82 \times 10^{-36} \mathrm{mr}
$$

This attractive force can be attributed to the cosmological constant, which translates conventional energy density that is not in the form of conventional baryonic mass. Moreover, the force of gravity, which varies in $r$, is active everywhere on the same basis as baryonic mass gravity. Note that such a force has never been detected around us because the cosmological constant is extremely small today $\left(\sim 10^{-54}\right)$. However, at the time of primitive galaxy formation, the cosmological constant was much greater $\left(\Lambda \sim 10^{-48}\right.$ at $\left.t \sim 0,5[G y]\right)$. Also, when we include the great galaxy or cluster radii, we will see that the cosmological gravity played a large part in galaxy rotation. For comparison purposes, let us calculate the ratio between the cosmological gravity and Newton's force for the solar system:

$$
\frac{F_{\Lambda}}{F_{G}}=\frac{\frac{\Lambda c^{2} m r}{6}}{\frac{G M m}{r^{2}}}=\frac{\Lambda c^{2} r^{3}}{6 G M}=\frac{\left(6,73 \times 10^{-54}\right)\left(2,99 \times 10^{8}\right)^{2}\left(149,6 \times 10^{9}\right)^{3}}{6\left(6,67 \times 10^{-11}\right)\left(1,98 \times 10^{30}\right)}=2,53 \times 10^{-24}
$$

For the earth, with small $\mathrm{g}_{\Lambda}$, the force assumes the following value:

$$
\mathrm{F}_{\Lambda}=\frac{\Lambda}{6} c^{2} r m=\frac{\left(6,73 \times 10^{-54}\right)\left(2,99 \times 10^{8}\right)^{2}\left(6378 \times 10^{3}\right)}{6} \mathrm{~m}=g_{\Lambda} m=6,39 \times 10^{-31}\left[\mathrm{~ms}^{-2}\right] m
$$

Note that the value for $\mathrm{g}_{\Lambda}$ is much too small to be detectable by current instruments. However, over the first billion years, let us calculate the ratio of the cosmological gravity to the force of gravity for the universe with a critical volumic mass of $3 \mathrm{H}^{2} / 8 \pi \mathrm{G}$ :

$$
\frac{F_{\Lambda}(1 G a)}{F_{G}(1 G a)}=\frac{\frac{\Lambda(1 G a) c^{2} m r}{6}}{\frac{G M m}{r^{2}}}=\frac{\Lambda(1 G a) c^{2} r^{3}}{6 G M}=\frac{\Lambda(1 G a) c^{2}}{8 \pi G \rho_{c r i}}=\frac{2,88 \times 10^{-49} c^{2}}{8 \pi G\left(1,79 \times 10^{-24}\right)} \sim 8,6
$$


Note that the attractive effect of cosmological gravity is huge and greatly surpasses that of gravity alone during the formation of great structures like galaxies. At $500[\mathrm{My}]$, the ratio was $\sim 34$. Figures 22 and 23 show the mean ratio $F_{\Lambda} / F_{G}$ for the time period starting at proton time $t_{\text {pr. Note }}$ that the cosmological gravity makes it possible for the great structures like galaxies to form much faster than simply under gravity. This notion of additional force to gravity could provide a possible explanation for the production of primitive black holes at the very beginning of the universe (6 $<\mathrm{z}<30$ ) (Lupi, Colpi, Devecchi et al., 2014). Indeed, the ratio $\mathrm{F}_{\Lambda} / \mathrm{F}_{\mathrm{G}}$ is $\sim 54$ aound 400 [My], which may accelerates the accumulation of mass beyond the Eddington limit.

Today, those effects are potentially limited to the great structures, such as galaxy clusters or superclusters, as it increases with an increase in radius. The time period when cosmological gravity was greater than gravity alone can be determined with:

$$
\begin{gathered}
F_{\Lambda} \geq F_{G} \\
\frac{\Lambda}{6} c^{2} r m \geq G \frac{M m}{r^{2}} \\
\Lambda \geq \frac{8 \pi G}{c^{2}} \rho \sim 1,87 \times 10^{-26} \rho
\end{gathered}
$$

Where $\rho$ is the volumic mass of matter in the zone concerned. For the entire universe at critical density, we get:

$$
\Lambda \geq \frac{8 \pi G}{c^{2}} \rho_{c} \geq \frac{3 H^{2}}{c^{2}}
$$

With the expression derived for the cosmological constant, we get the following expression, which yields the cosmic time at which cosmological gravity was greater than gravity force alone: 


$$
\alpha H^{4}-\varepsilon H^{3} \geq \frac{3 H^{2}}{c^{2}}
$$

With the values for $\alpha$ and $\varepsilon$ already obtained, cosmic time is found to be:

$$
t_{\text {cosmic }} \leq 2,89[G y](\mathrm{z} \geq 3,77)
$$

Therefore, cosmological gravity is the dominant force beyond gravity alone for a time period of $\sim 2,9[\mathrm{~Gy}]$.

This cosmological gravity force may have an impact on the different concepts used in cosmology as the Eddington limit, the Jeans radius. For the first $\sim 3[G y]$, the values obtained from the concepts can be adapted using the adapted Newton gravitation constant $G^{\Lambda}$ to take into account this cosmological force of a structure mass $\mathrm{M}$ and radius $\mathrm{r}$ by substituting $G$ with the adapted one.

$$
G^{\Lambda}(H)=\left(1+\frac{\Gamma H^{4} r^{3}}{M}\right) G
$$

With: $\Gamma=\frac{c^{2} k_{\Lambda}}{6 G} \sim 6,47 \times 10^{43}\left[k g s^{4} m^{-3}\right]$

This expression of $G^{\Lambda}(H)$ was proposed repeatedly by many authors as part of a family of models called: Dark matter, dark energy dynamical scalar field (quintessence) (Amendola et al, 2018). The general form of the equation proposed is:

$$
G(\alpha)=\left(1+2 \alpha^{2}(\varphi)\right) G
$$

The value mentioned for $\alpha$ compatible with the $\mathrm{CMB}$ is $(0<\alpha<0,06)$. We find this value of $\alpha$ for the MW. 


$$
\alpha(\varphi)=\sqrt{\left(\frac{\Gamma H_{0}^{4} r^{3}}{2 M}\right)} \sim \sqrt{\left(\frac{6,47 \times 10^{43} \times 2,29 \times 10^{-18^{4}} \times 6,478 \times 10^{20} 0^{3}}{2 \times 2,97 \times 10^{41}}\right)} \sim 9 \times 10^{-4}
$$

According to the author, while that force is negligible today on our scale, it was central to the formation of our universe and the great structures within it.

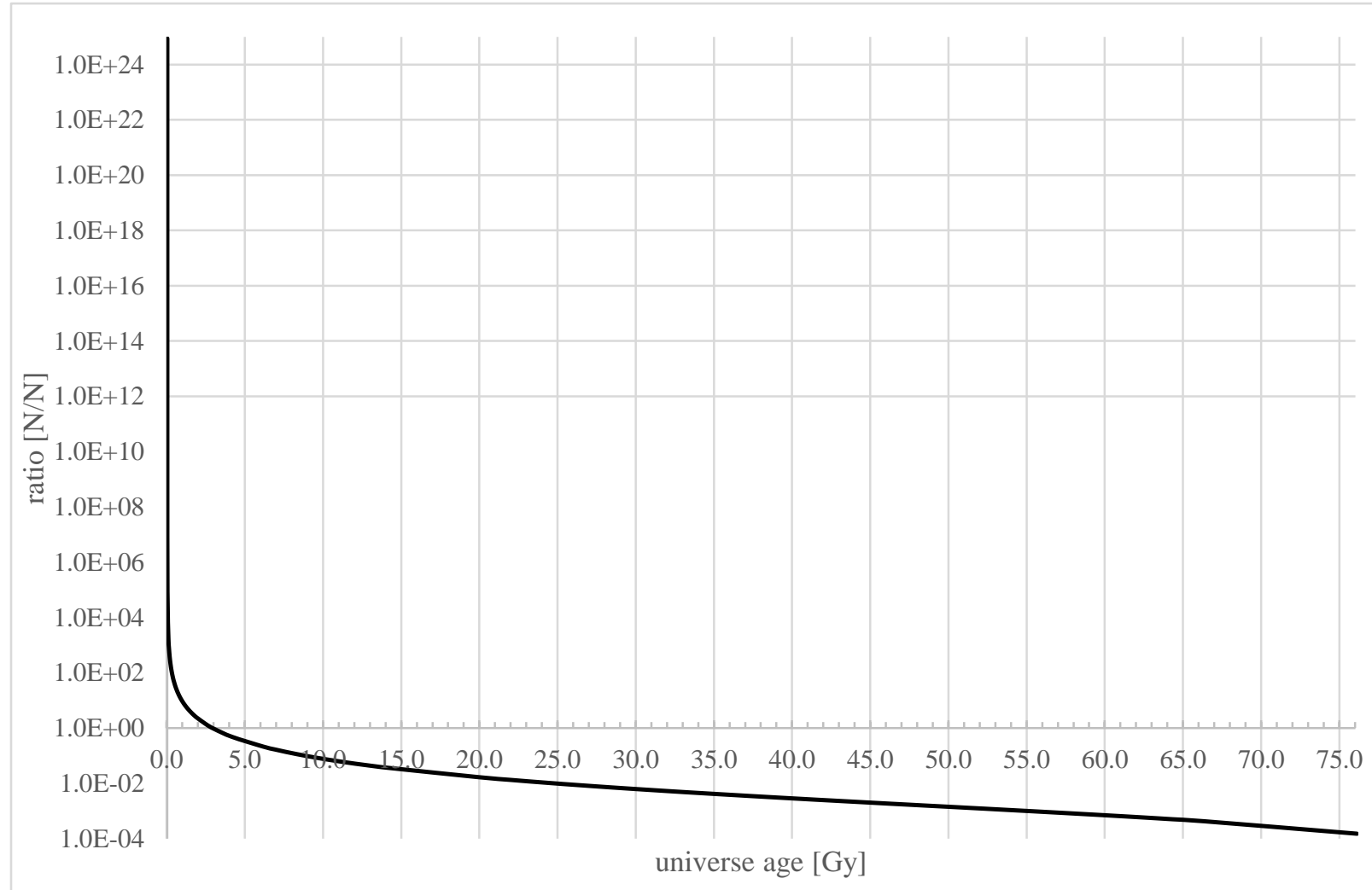

Figure 22: Ratio of $F_{\Lambda} / F_{G}$ from $1 t_{p}$ to 76,1 [Gy] 


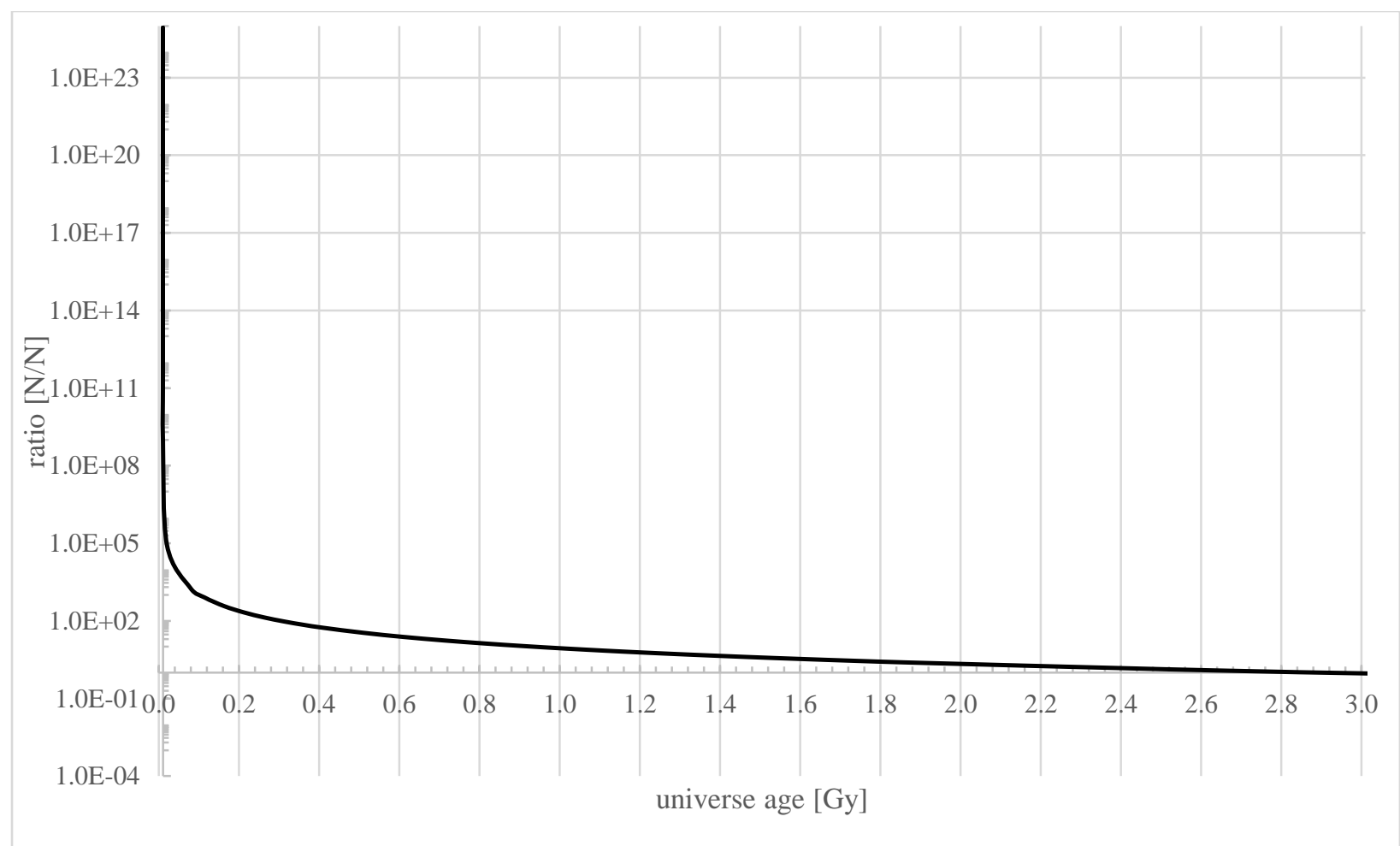

Figure 23: Ratio of $F_{\Lambda} / F_{G}$ from $1 t_{p}$ to 3 [Gy]

\section{Attractive cosmological gravity, $\mathbf{F}_{\Lambda}$, and galaxy rotation (simplified model)}

The formation and evolution of galaxies is a very complex field of study, and the associated mechanisms have not yet been fully interpreted. Indeed, the number of phenomena in play during galactogenesis, such as supplemental forces to gravity, the birth of stars and internal structures, energy dissipation effects, and the quantity and type of neighbouring matter being absorbed are only some of the factors involved in galaxy formation, North (2011). A relatively complete model has been put forth by Martig et al. (2018), which assumes the presence or existence of dark matter that is as much subject to gravity (Kepler) as baryonic matter. In this article, as aforementioned, we do not consider the existence of dark matter, but rather energy at time $t$ (non-massive) and the mass-energy equivalence acting through the cosmological constant. This has already been 
discussed by Gessner (1992), where the cosmological constant did not vary during the formation process of the structures. This may be due to the lack of a predictive model for $\Lambda(\mathrm{t})$, which hinders the simulation of velocity profiles for structures with either small or great radii. With such a predictive model, the impact of this attractive force on galaxy rotation can be seen. We do know the values of the cosmological constant, $\Lambda$, at the time of primitive galaxy formation (1-2 [Gy]). We can calculate that attractive force and see its effects on the rotation of some galaxies. Put simply, for a given circular rotation orbit, the tangential rotation speed of a mass is expressed through the balance of the main forces considered in the model: gravity and cosmological gravity via massenergy equivalence:

$$
\begin{gathered}
F_{c}=F_{G}+F_{\Lambda} \\
m \omega^{2} r=G m\left(\frac{M}{r^{2}}+\frac{\Lambda c^{2} r}{6 G}\right) \\
v_{t}^{2}=G\left(\frac{M}{r}+\frac{\Lambda c^{2} r^{2}}{6 G}\right)
\end{gathered}
$$

Note that cosmological attractive force associated with $\Lambda$ is supplemental to conventional gravity (baryonic). Moreover, that force cannot be attributed to negative masses or so-called "dark" unobservable forces. In fact, the denominator of the second term is not the inverse of the radius, which confirms that the force is not due to the effects of mass as such, but to a mass-energy equivalence associated with $\Lambda$. Finally, because that force is relative to $\Lambda$, which is relative to the age of the universe, the rotation profile of masses like galaxies is in turn relative to time from the standpoint of forces in play. In other words, the rotation profile should take into consideration the evolution of $\Lambda$ as the galaxy absorbs matter over time. The actual process behind the action of this 
cosmological gravity, $\mathrm{F}_{\Lambda}$, on the rotation dynamics of galaxies is complex, as it is relative to both time and the radius of any given galaxy:

$$
v_{t}^{2}(r, t)=G\left(\frac{\rho(r, t) V(r, t)}{r(t)}+\frac{\Lambda(t) c^{2} r(t)^{2}}{6 G}\right)
$$

Solving this equation is beyond the scope of this paper because we would need to know the density profile of matter in the galaxy relative to time, $t$, meaning the formation mechanismes of that galaxy from a dynamic standpoint (mass accumulation process and rate). A simulator like Millenium could derive that term associated with $F_{\Lambda}$. However, in this paper, we want to demonstrate that assuming the existence of dark matter is not necessary at first to describe the galaxy formation process and rotation curves as we see them today. To do so, the galaxy formation process can be simplified by assuming that mass accumulates according to a simple function of time, and that $\Lambda(\mathrm{t})$ also evolves according to time (bottom-up model). The simplified equation of galaxy rotation has three terms, the effects associated with the bulbe, or denser central area, and with the disc around the central area, and the effects of $\Lambda(t)$ at formation time $t$ and radius $r(t)$. At first, we will not consider dark matter, also called halo mass, although such dark baryonic mass (non-radiating) surely must exist within galaxies. We will see that for some galaxies, such as M33, the observable mass (luminous) is not sufficient to explain the observed rotations, meaning that we have to assume the probabal existence of baryonic dark masses.

The time at which a galaxy started to form is important because it influences the effective value of $\Lambda$. Then, the formation time of the galaxy is just as important (acceleration rate of the mass), since this yields the total variation of $\Lambda$ on the rotation process. To initially demonstrate the effects of force $\mathrm{F}_{\Lambda}$ on galaxy rotation, let us find an expression of rotation speed relative to time: the time at which the galaxy started to form, $\mathrm{t}_{\mathrm{i}}$, the total formation time of the galaxy, $\mathrm{t}_{\mathrm{T}}$, with variable force, 
$\mathrm{F}_{\Lambda}$, acting during that formation time, $\mathrm{t}_{\mathrm{T}}-\mathrm{t}_{\mathrm{i}}$. For the masses of the bulbe and disc, we get a simplified expression:

$$
\begin{gathered}
M_{T}(r, t)=M_{b}(r, t)+M_{d}(r, t) \\
M_{T}(r, t)=\frac{V_{b}(r, t)}{V_{b_{T}}} M_{b}+\frac{V_{d}(r, t)}{V_{d_{T}}} M_{d}=\frac{r(t)^{3}}{r_{b}^{3}} M_{b}+\frac{\left(r(t)^{2}-r_{b}^{2}\right)}{\left(r_{T}^{2}-r_{b}^{2}\right)} M_{d}
\end{gathered}
$$

Where:

$0 \leq r(t) \leq r_{b}$, for the bulbe

$r_{b} \leq r(t) \leq r_{T}$, for the disc

$r_{b}$ : bulbe radius determined at the end of galaxy formation

$r_{T}$ : disc and bulbe radii determined at the end of galaxy formation

$M_{b}$ : bulbe mass determined at the end of galaxy formation

$M_{d}$ : disc mass determined at the end of galaxy formation

A simple law can be used to calculate mass accumulation at a constant rate:

$$
r(t)=\alpha t=\frac{t}{\left(t_{T}-t_{i}\right)} r_{T}
$$

Where

$t_{i} \leq t \leq t_{T}$, formation time of the galaxy

$\alpha$ : galaxy radius growth rate (accumulation) 
For the mass, we get:

$$
M_{T}(r, t)=\frac{\alpha^{3} t^{3}}{r_{b}^{3}} M_{b}+\frac{\left(\alpha^{2} t^{2}-r_{b}^{2}\right)}{\left(r_{T}^{2}-r_{b}^{2}\right)} M_{d}
$$

For rotation speed, we get:

$$
\begin{gathered}
v_{t}^{2}=G\left(\frac{M}{r}+\frac{\Lambda c^{2} r^{2}}{6 G}\right)=\frac{G}{r}\left[\frac{\alpha^{3} t^{3}}{r_{b}^{3}} M_{b}+\frac{\left(\alpha^{2} t^{2}-r_{b}^{2}\right)}{\left(r_{T}^{2}-r_{b}^{2}\right)} M_{d}\right]+\frac{k_{\Lambda} c^{2} \alpha^{2} t^{2}}{6\left(t+t_{i}\right)^{4}} \\
v_{t}^{2}=G\left[\frac{\alpha^{2} t^{2}}{r_{b}^{3}} M_{b}+\frac{\left(\alpha^{2} t^{2}-r_{b}^{2}\right)}{\alpha t\left(r_{T}^{2}-r_{b}^{2}\right)} M_{d}\right]+\frac{k_{\Lambda} c^{2} \alpha^{2} t^{2}}{6\left(t+t_{i}\right)^{4}}
\end{gathered}
$$

Where

$t_{i} \leq t \leq t_{T}$, formation time of the galaxy

$t_{i} \leq t_{b} \leq t_{B}$ : formation time of the galaxy bulbe

$t_{B} \leq t_{d} \leq t_{T}$ : formation time of the galaxy disc

$t_{i}$ : age of the universe at the time of galaxy formation to calculate $\Lambda(\mathrm{t})=\frac{k_{\Lambda}}{\left(t_{i}+t\right)^{4}}=k_{\Lambda} H^{4}$, with:

$$
k_{\Lambda}=\left[\frac{32 \pi G \sigma C_{1}^{4}}{c^{5}}\right]=\left[\frac{32 \pi G \sigma T_{\Omega}^{4} t_{\Omega}^{4}}{c^{5}}\right]=2,88 \times 10^{17}\left[s^{4} m^{-2}\right]
$$

In the above equation for $\mathrm{v}_{\mathrm{t}}$, the first term is for the attraction of the bulbe on the rotating mass, the second, for the attraction of the disc, and the third, for the attraction of force $F_{\Lambda}$ due to the cosmological constant through the residual mass-energy equivalence of the universe at the beginning of formation, $\mathrm{t}_{\mathrm{i}}$, of the galaxy acting throughout formation time, $\mathrm{t}_{\mathrm{T}}-\mathrm{t}_{\mathrm{i}}$. This equation contains the essential elements for predicting the rotation curve of the luminous mass of galaxies. Force $\mathrm{F}_{\Lambda}$ decreases over time, or the age of the universe, but one must consider that the prevailing 
conditions of galaxy formation are still present in the space-time continuum of that galaxy. In other words, we will see that, in simulations of the rotation of some galaxies, the time at which mass started to accumulate is crucial for the development of the type of rotation because cosmological gravity varies like $\mathrm{t}^{4}$, or inversely with the age of the universe during the formation of that galaxy. This means that the type of rotation curve (concave or convex $^{-}$) lets us know, in part, whether the galaxy was formed in the early days of the universe, or later (concave $=$ older; convex $=$ younger). So, a weaker cosmological gravity should lead to Keplerian rotation, or a convex curve. Finally, we have assumed a very simple galaxy radius growth rate that is linear over time. Other, more realistic models can be introduced in the equation to better illustrate the generic growth of an isolated galaxy. Of course, the impacts of galactic collisions and agglomerations are not considered here.

\section{Mass rotation equation and tangential velocity}

The rotation equation involves five parameters to determine, at first glance, the rotation of a galaxy assuming that it has not undergone severe transformations, such as collisions with other massive bodies. In this study, we propose a bottom-up approach with the following parameters:

- Start time of bulbe formation (beginning of accumulation)

- Galaxy formation time (end of major accumulation)

- Formation time of the bulbe (the disc begins to form)

- Bulbe mass accumulated during bulbe formation time

- Disc mass accumulated during disc formation time

The actual mass distribution and radial velocity of galaxies is complex, other parameters have to be considered, such as the presence of gases, and small neighbouring structures or more massive 
structures nearby (like other galaxies), etc. However, we will see that the equation requires careful consideration to significantly reduce the need to consider the dark matter halo (invisible) to explain rotation speeds. Dark matter is not considered in this model, but we do consider non-luminous baryonic matter.

\section{MW, S(B)bc I-II}

Many studies have been conducted to try and determine the velocity profile and mass of the MW. Several variations of the luminous mass have been reported, and many authors include an estimation of the dark matter halo to validate certain observations or conclusions. Indeed, according to a number of studies, the total mass of the MW can vary by as much as a factor of seven $\left(5,8 \times 10^{11} \mathrm{M}_{\odot}<\mathrm{M}_{\mathrm{t}}<4,5 \times 10^{12} \mathrm{M}_{\odot}\right)$ (Karachentsev and Kashibadze, 2006) ; (Peñarrubia, Ma, Walker and McConnachie, 2014), depending on the presence or absence of dark matter. Moreover, the accepted structure of the MW includes a $\sim 1[\mathrm{kpc}]$ (luminous mass of $1,7 \times 10^{10} M_{\odot}$ ) center with radio emissions, a thick disc of $\sim 1$ [kpc] (luminous mass of $1 \times 10^{9} M_{\odot}$ ), a thin disc of $\sim 20[\mathrm{kpc}]$ (luminous mass of $6 \times 10^{10} M_{\odot}$ ), and several spiral arms. Also, the MW may have collided with Andromeda in the past, but this is an unverified assumption. In other words, the velocity profile of the MW may have been disrupted by past events that the velocity prediction model cannot consider due to a lack of relevant data.

Figures 24 and 25 show an approximate evolution for the mean rotation curve of the MW from the time formation began $\left(\mathrm{t}_{\mathrm{i}}=0,181[\mathrm{~Gy}]\right.$ and $\left.\mathrm{t}_{\mathrm{t}}=0,32[\mathrm{~Gy}]\right)$ until today $\left(\mathrm{t}_{\Omega}=76,1[\mathrm{~Gy}]\right)$. The galaxy's mass is considered constant after its main formation. Realistically, however, accumulation is a continuous process. In this model, we will see that the main formation of galaxies seems to have 
occurred around the beginning of the universe $(<1,5[\mathrm{~Gy}])$, and that accumulation progressively decreases thereafter, even though the intrinsic motion of galaxies continues over time and events (collisions, restructurings, amalgamations). In fact, the early formation of structures like massive black holes and galaxies $(<500[M y])$ could be made possible by a direct collapse mechanism (Natarajan, Pacucci, Ferrara et al., 2017). Recently, a team discovered a candidate galaxy, SPT0615-JD, at $\mathrm{z} \sim 10-11$, that may have existed around 400 [My] after the beginning (Salmon, Coe, Bradley et al., 2018). Also, another team reported the lens-effect observation of a star dating back to earlier than 250 [My] in galaxy MACS1149-JD1 (Hashimoto, Laporte, Mawatari et al., 2018).

The simulation process is as follows: The primitive formation of the galaxy is determined by trial and error using the aforementioned five-paremeter equation. Then, the galaxy undergoes expansion of the universe on a scale factor until today. The simulation can be extended into the MW's future. At the beginning, the MW had a radius of about $r=0,34$ [kpc]. This is smaller than stated by Martig et al, (2018), a disc dimension derived from dynamic galaxy simulations ( $\mathrm{r}_{\text {core }} \sim$ 1,79 [kpc], case G10). However, taking into consideration the appropriate scale factor for the simulations by Martig $(\mathrm{z}=2)(4,6[G y] / 13,8[G y])$, the starting dimension of the galaxy disc for case G10 is reduced to $\sim 0,59$. Rotation speed of the center was quite high, around $1100\left[\mathrm{kms}^{-1}\right]$. Then, the MW increased in size by a scale factor and speeds dropped. Around 6 [Gy], its diameter was around 1,6 [kpc]. 


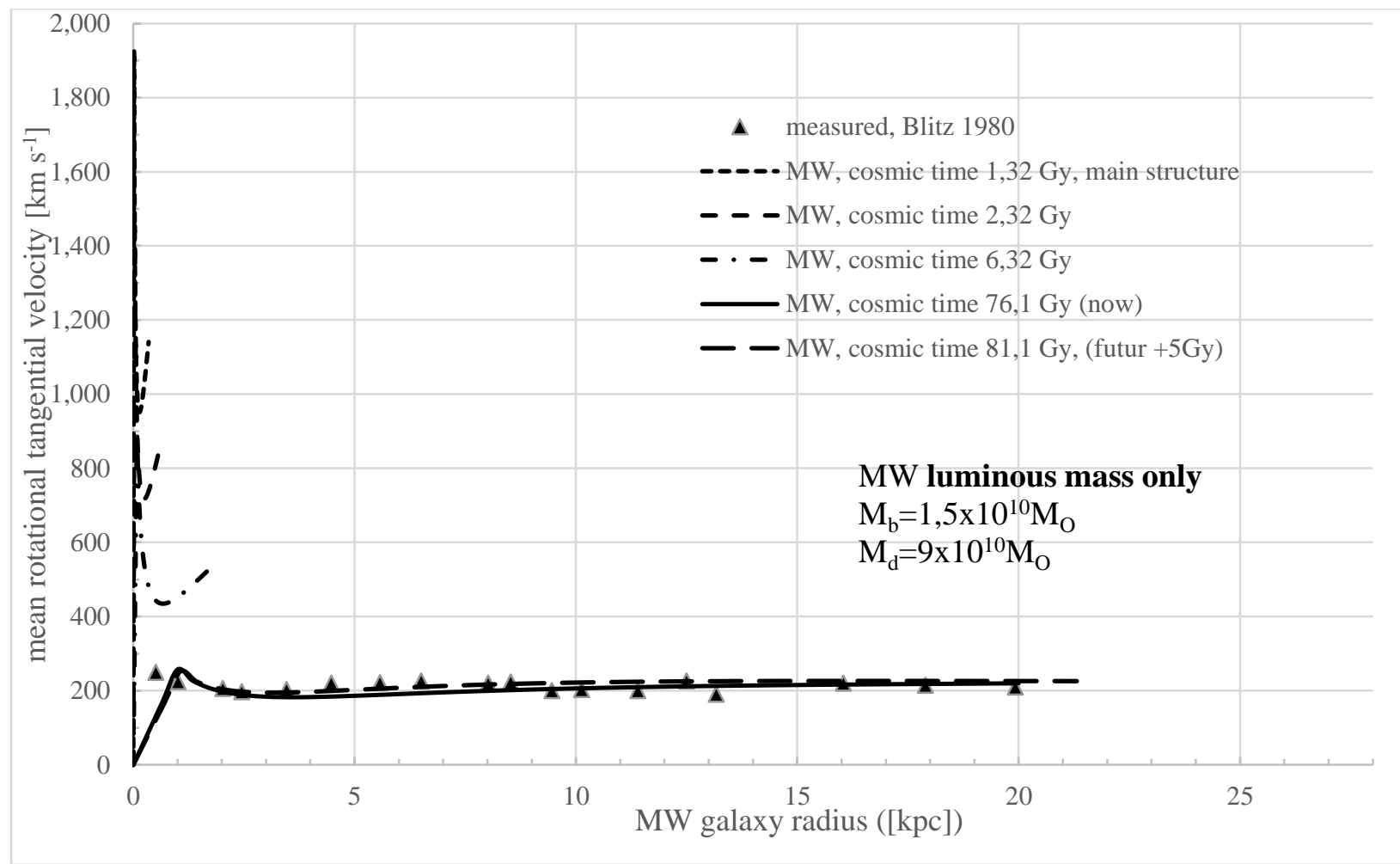

Figure 24: MW rotational and size evolution since formation to 81,1 [Gy]

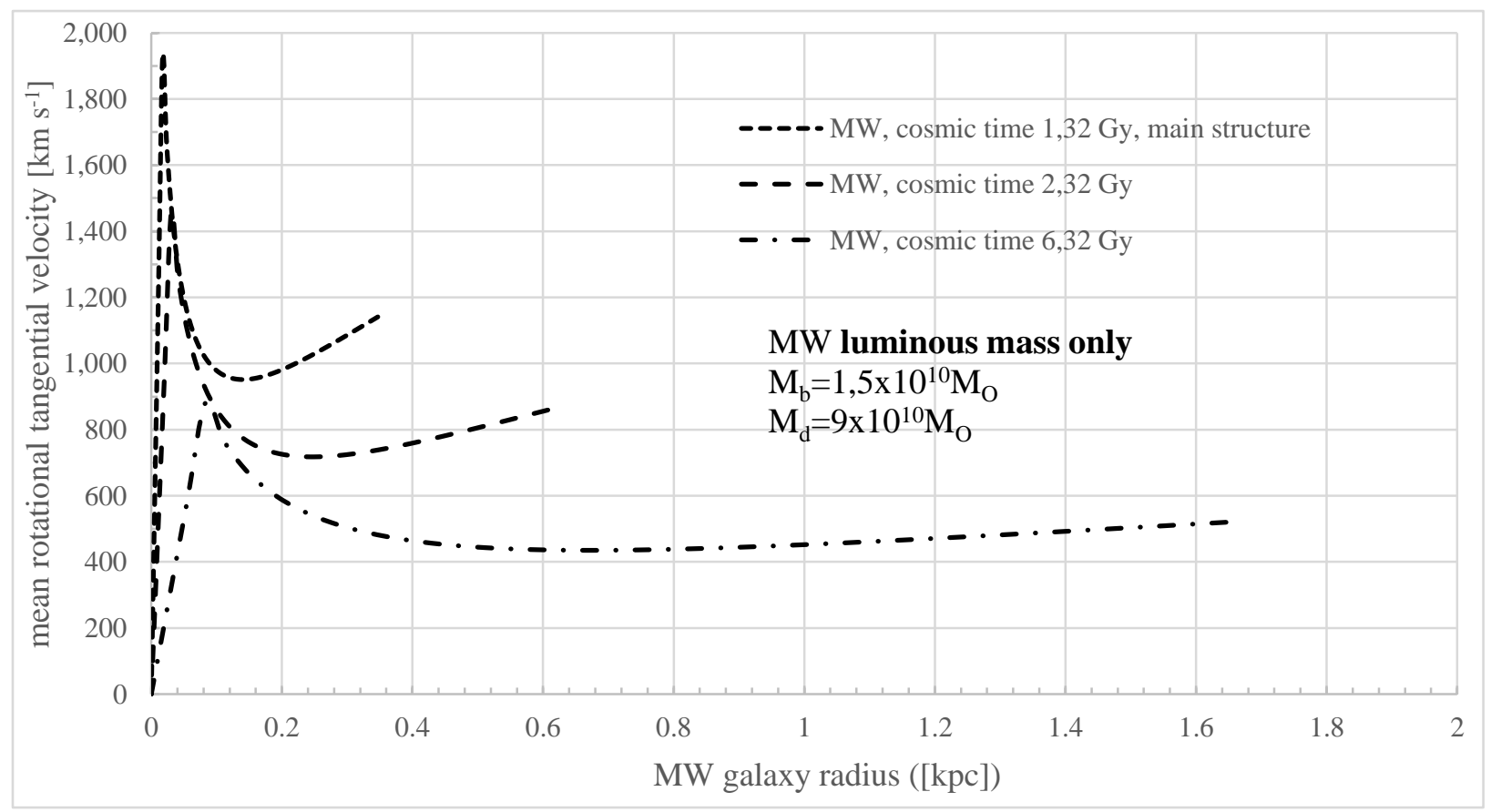

Figure 25: MW rotational and size evolution since formation to 6 [Gy] 
Figure 26 shows three velocity profiles with observed masses and the three remaining parameters of the equation: $t_{i}, t_{T}$ and $t_{b}$, along with cosmological gravity, $F_{\Lambda}$, calculated at the time the MW was formed. The first curve is observed masses and Kepler rotation speeds only. Note that the speeds for large radii are not well represented. The second curve is for a galaxy formation beginning earlier, around 150 [My], but longer formation time of 450 [My]. Note that the speeds are better represented than in the previous graph but are generally still too low. The last curve is the formation of the MW, which may have begun around $180[\mathrm{My}]$, with the main accumulation lasting some 320 [My]. Here, the speeds do not follow the measured profile closely, but the values are in the proper order of magnitude for great radii. Note that speed variations, around 10 [kpc], are not well represented, which could indicate a much more complex accumulation process than the linear model used (change of rate). As concerns the beginning of formation, around 180 [My], observations have shown that stars like HE 1523-0901 and HD 140283 are as old as 600 [My] and even 150 [My] after the beginning (Frebel, Christlieb, Norris et al., 2008). HE 1523-0901 is located 7500 light years away from Earth $(2,29[\mathrm{kpc}])$. If we estimate the position of the sun to be $\mathrm{r}=8.5+/-$ $0.5[\mathrm{kpc}]$, the formation of a star at that position is possible:

$$
\begin{gathered}
\mathrm{t}>t_{i}+\frac{r_{\odot}+/-0,5}{\alpha}+/-\frac{2,29}{\alpha}=181[M y]+\frac{8.5+/-0,5 k p c}{\frac{20 k p c}{320 M y}}+/-\frac{2,29 k p c}{\frac{20 k p c}{320 M y}} \\
\mathrm{t}=181+136 \pm 8[\mathrm{My}] \pm 37[\mathrm{My}]=317 \pm 45[\mathrm{My}]
\end{gathered}
$$

That star was formed about 500-600 [My] after the beginning, which fits with the current MW formation model. Indeed, this leaves $\sim 138$ to 238 [My] for that star to form at that position. As for HD 140283, it may have started to form about 150 [My] after the beginning, which could mean that the formation of the MW began earlier than the assumed 181 [My]. However, a lot of uncertainty remains about the formation of that star very close to the sun $(0,06[\mathrm{kpc}])$. In short, the 
amount of unobservable mass could be quite small in the MW, meaning that rotation speeds could be largely due to cosmological gravity in play during formation, or 180 [My] after the beginning.

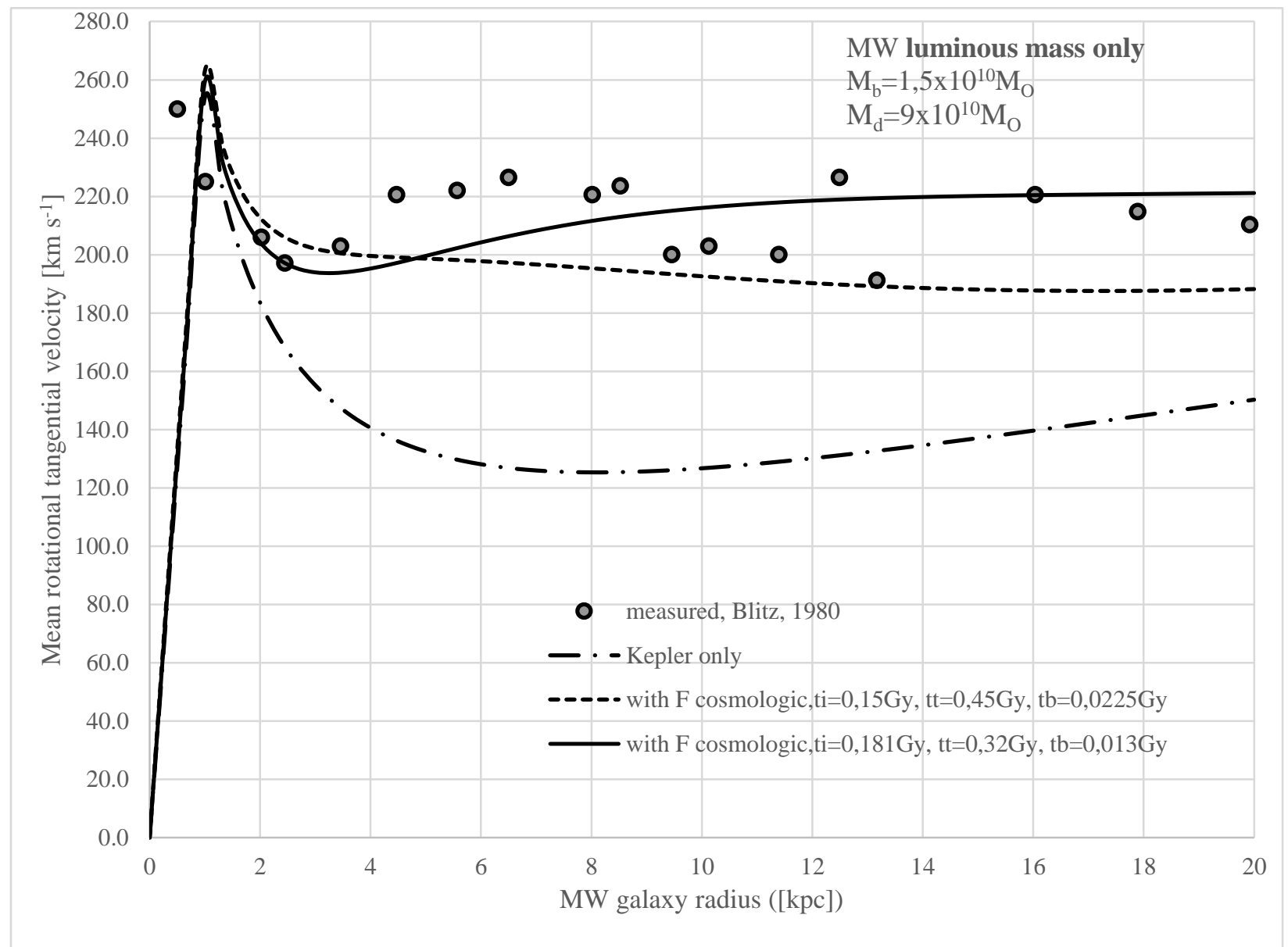

Figure 26: MW rotational velocities

\section{M33 (SA(s)cd) (of the triangle)}

Studying M33 to explain the radial velocity equation is an arbitrary choice, but we need a galaxy that has apparently not collided with another galaxy in the past, and which contains a large amount of dark matter. In fact, this galaxy is reportedly $85 \%$ dark matter (Corbelli, 2003). If the dark matter is removed, the following luminous masses remain:

$$
M_{\text {Tvisibe }} \sim 0,15 M_{T} \sim 0,15\left(6 \times 10^{10} M_{\odot}\right) \sim 9 \times 10^{9} M_{\odot}
$$


To avoid making too many speculative simulations regarding center and disc masses, we chose the following values as constants:

$$
\begin{aligned}
& M_{d \text { visibe }} \sim 9 \times 10^{9} M_{\odot} \\
& M_{\text {b visibe }} \sim 5,0 \times 10^{8} M_{\odot}
\end{aligned}
$$

Figure 27 shows five rotation curves for M33, derived from the three remaining parameters of the equation: $t_{i}, t_{T}$ and $t_{b}$, along with the rotation curve for the luminous mass only (Kepler baryonic, $\mathrm{F}_{\Lambda}=0$ ). Note that the rotation of this galaxy is not as well represented for the greater radii. The beginning of formation is assumed to be $\mathrm{t}_{\mathrm{i}}=0,16$ to 0,2 [Gy]. Note also that a start time closer to the beginning increases the concave nature of the velocity profile, due to the stronger effect of the cosmological constant and the longer formation time, which flattens the velocity profile, as the cosmological constant decreases more sharply in remote areas with large radii. Further, the luminous mass is not sufficient to explain the rotation of the outer radius, as the speeds decrease sharply beyond $9[\mathrm{kpc}]$. This confirms the presence of dark matter (baryonic non-luminous) in this galaxy because cosmological gravity alone is not enough to accurately simulate the rotation. To determine the effects of non-luminous matter, the last curve represents a mass total that is six times greater than the estimated luminous mass $\left(5,59 \times 10^{10} M_{\odot}\right)$. Note the strong correspondence between the estimated and measured speeds, clearly showing the existence of non-uminous matter in M33 and similar galaxies. 


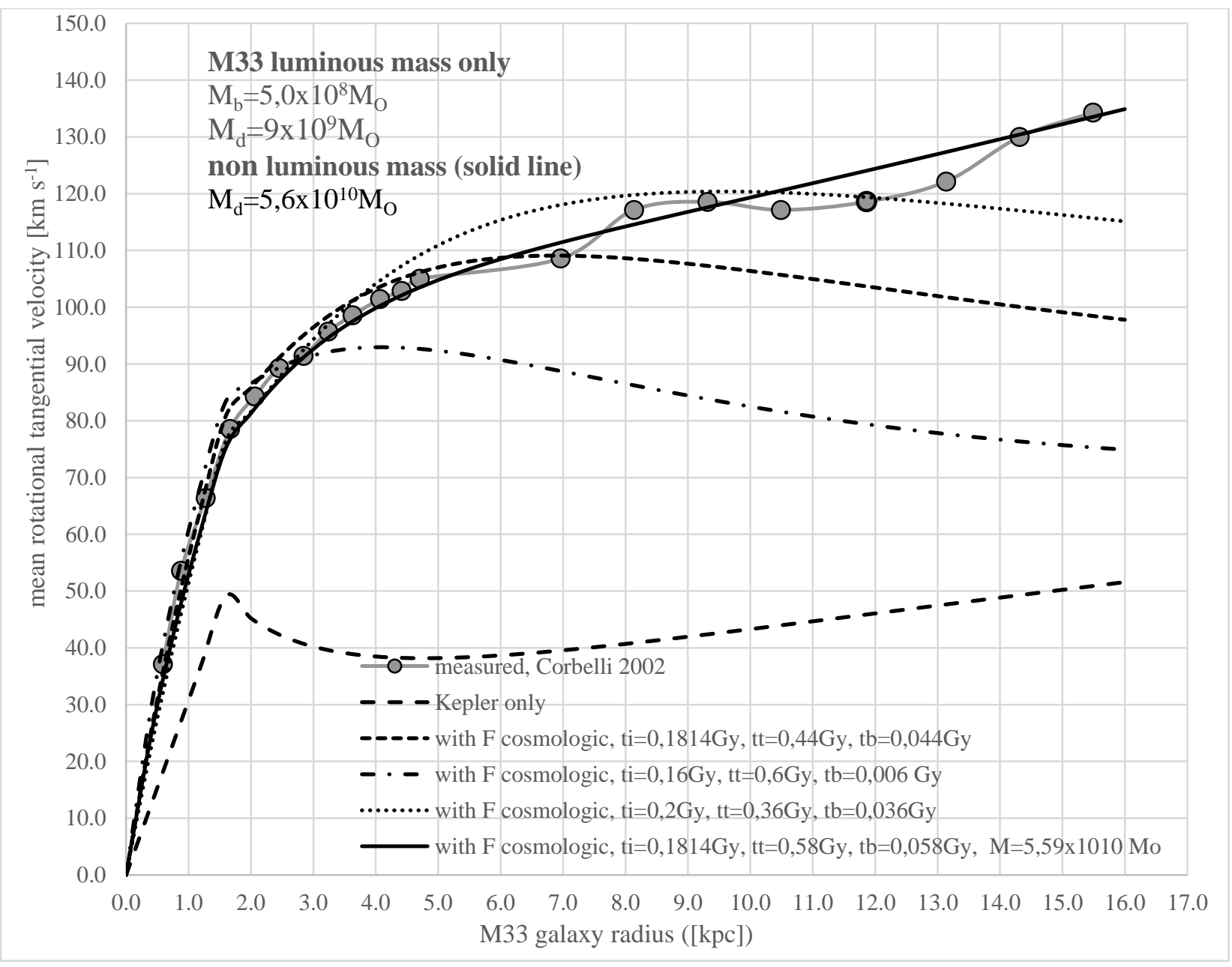

Figure 27: M33 rotational velocities

\section{UGC12591, S0/Sa (Pegasus)}

Galaxy UGC12591 was chosen to demonstrate the significant effects of cosmological gravity, $\mathrm{F}_{\Lambda}$, on the formation and faster rotation speeds of early galaxies. Careful studies by Giovanelli et al. (1986) and Xinyu Dai (2012) show very large amounts of dark matter (84\%). Removing the dark matter from the reported total mass $\left(\sim 2,7 \times 10^{12} M_{\odot}\right)$ yields the following luminous mass:

$$
M_{\text {Tbaryon }} \sim 0,17 M_{T} \sim 0,17\left(2,7 \times 10^{12} M_{\odot}\right) \sim 4,6 \times 10^{11} M_{\odot}
$$


Center mass is not specified as such. The rotation curve shows that the center mass should be greater than the disc mass to be able to closely simulate the observed rotation speeds:

$$
\begin{aligned}
& M_{b \text { visibe }}=0,69 M_{\text {visibe }} \sim 0,69\left(4,6 \times 10^{11} M_{\odot}\right) \sim 3,18 \times 10^{11} M_{\odot} \\
& M_{d \text { visibe }}=0,31 M_{\text {visibe }} \sim 0,31\left(4,6 \times 10^{11} M_{\odot}\right) \sim 1,42 \times 10^{11} M_{\odot}
\end{aligned}
$$

Figure 28 shows two rotation curves for USG 12591, based on the three remaining parameters of the equation: $t_{i}, t_{T}$ et $t_{b}$ and the rotation curve of the luminous mass only (Kepler baryonic, $F_{\Lambda}=0$ ). The observed speed curve is relatively downward linear, indicating an early formation time. Indeed, with an adjustment of the three parameters, we see that the formation of that galaxy began around $176[\mathrm{My}]$ and continued for about $280[\mathrm{My}]$. Note here that this formation period is called primitive as this is when most of the mass is accumulated. Of course, the evolution of galaxies is dynamic and continuous. Finally, the rotation curve shows that this galaxy's luminous mass is sufficient to generate the observed rotation speeds. The luminous mass of this galaxy is $\sim 4,4$ times greater than that of the MW, and its center mass alone is $\sim 21$ times greater, which partly explains the great rotation speeds starting in the first $5[\mathrm{kpc}]$ of the radius. 


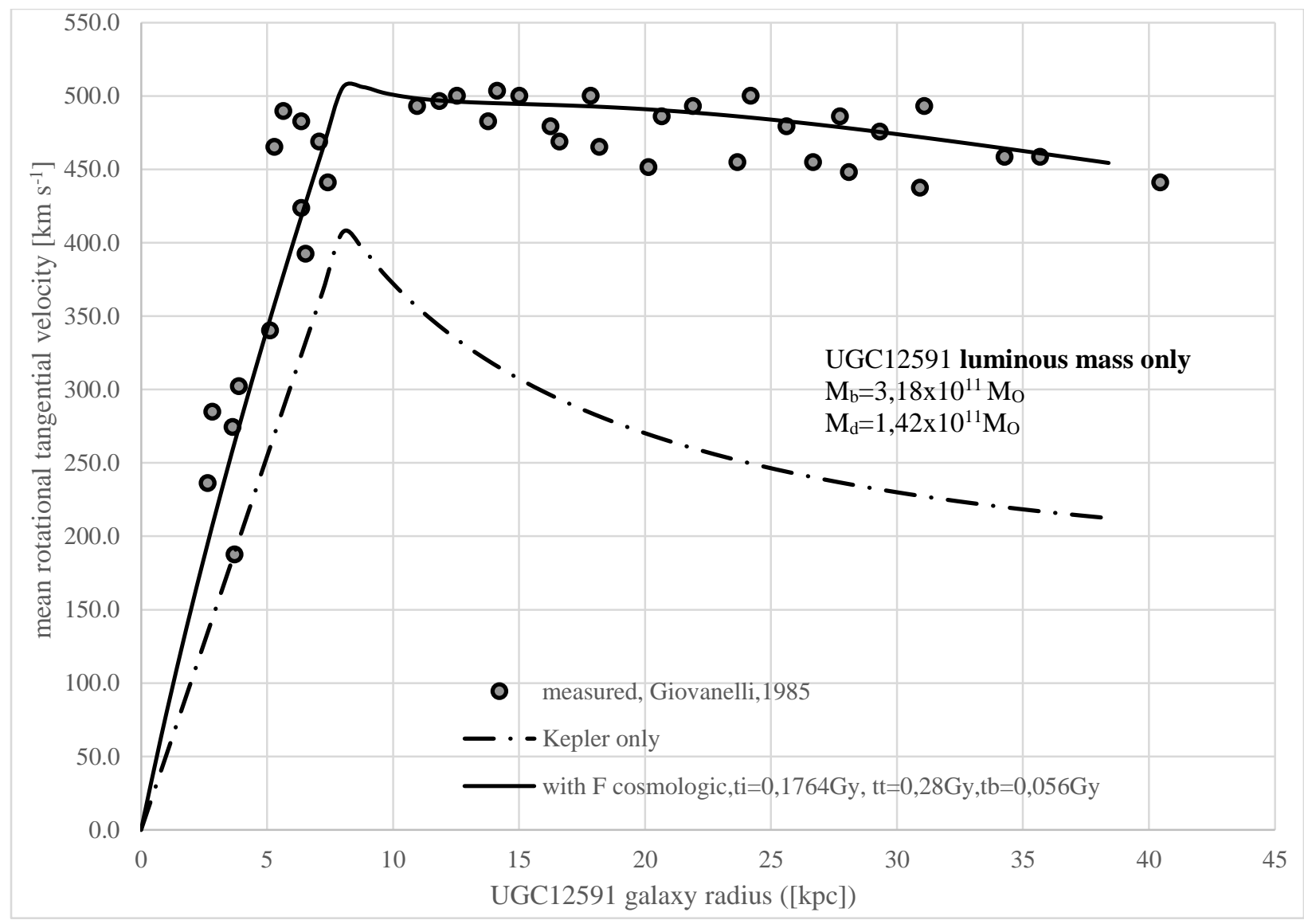

Figure 28: UGC12591 rotational velocities

\section{NGC3198, Sc C}

This spiral galaxy has been the object of many studies to determine its velocity profile and the mass of hydrogen gas outside its planar disc (Gentile, Józsa, Serra et al., 2013).

Figure 29 shows three velocity profile curves. The first shows the estimated luminous mass, $1,08 \times 10^{10} M_{\odot}$ and the three remaining parameters of the equation: $t_{i}, t_{T}$ and $t_{b}$, along with cosmological gravity, $\mathrm{F}_{\Lambda}$, calculated at the formation time of the galaxy. Note that its mass is too small to generate rotation speeds beyond $15[\mathrm{kpc}]$, even in consideration of the cosmological gravity in play at the beginning of formation, around $165[\mathrm{My}]$. The presence of a substantial non luminous matter halo is necessary here to explain the rotation speeds at the outer edges. For 
comparison purposes, the second curve shows the amount of mass added to the luminous mass to justify the rotation speeds, which is 16 times greater than the estimated mass $\left(1,78 \times 10^{11} / 1,08 \times 10^{10}\right)$. The last curve shows only the Kepler speed for the observed mass only.

This galaxy probably began to form around $181[\mathrm{My}]$ and the main accumulation may have lasted nearly $1[\mathrm{~Gy}](880[\mathrm{My}])$, if the amount of lacking mass is considered. Note that non-luminous mass must be considered here, which tends to confirm that non luminous mass can make up significant proportions of galaxies, even when cosmological gravity is in full force.

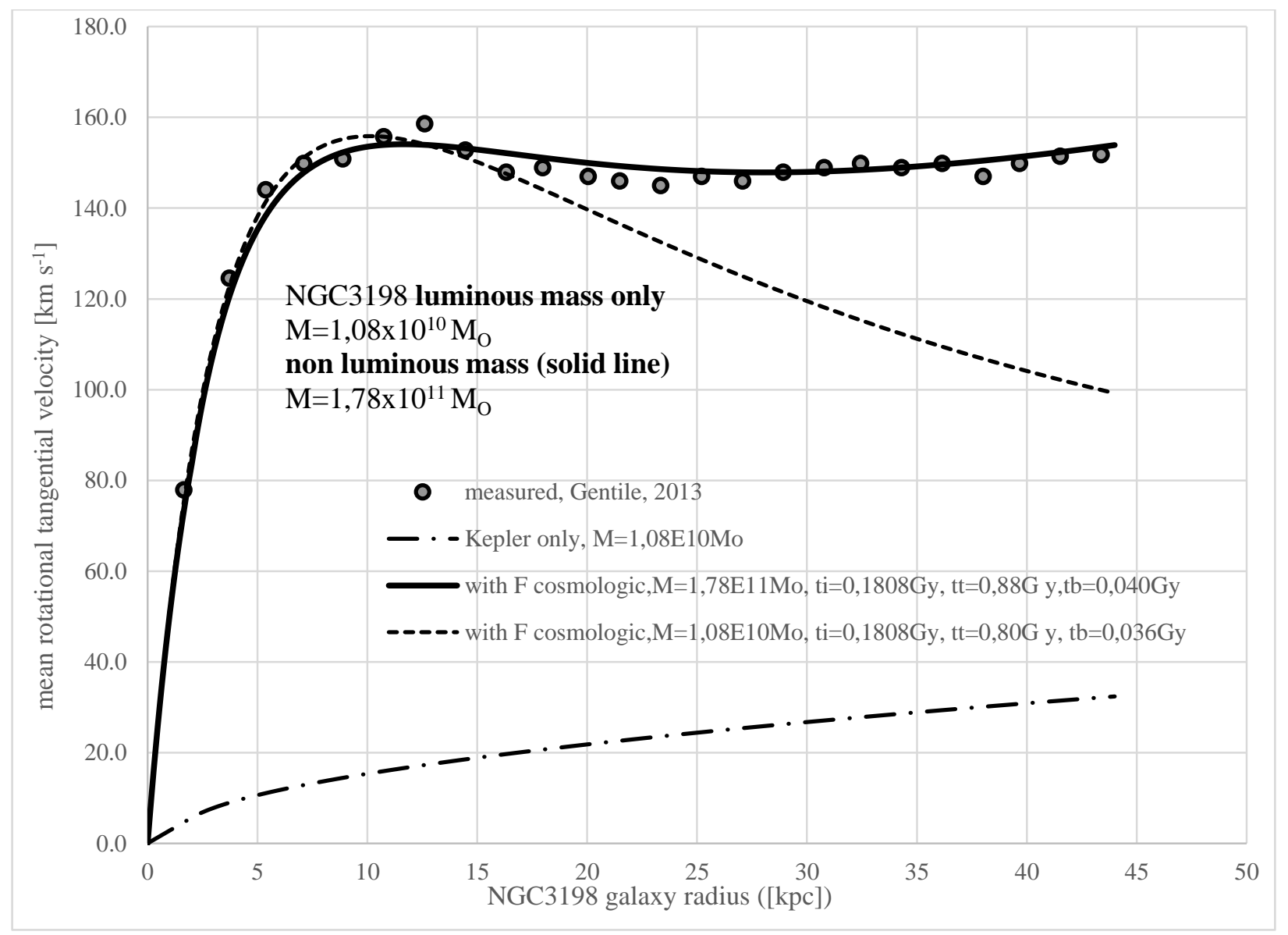

Figure 29: NGC3198 rotational velocities 


\section{UGC2885, Sc D}

One of the largest of spiral galaxies observed to date has been the object of many studies. Figure 30 shows two velocity profile curves. The first for the estimated observable mass, $2 \times 10^{12} \mathrm{M}_{\odot}$ and the three remaining parameters of the equation: $t_{i}, t_{T}$ and $t_{b}$, along with the cosmological gravity, $\mathrm{F}_{\Lambda}$, calculated at the formation time of the galaxy. Note that the mass here is sufficient to generate the rotation speeds. The peak rotation velocity near the center is accurately predicted, but the measured peak is more spread out. The value used for the center mass, $4 \times 10^{10} \mathrm{M}_{\odot}$, is in the same order of magnitude as the $10^{10} \mathrm{M}_{\odot}$ estimated by Gentile (2013). Velocities at the outer radius are greater than those measured and quasi-constant at $298 \mathrm{~km} \mathrm{~s}^{-1}$. However, the mass accumulation model we use is a very simple one, meaning that the galaxy's mass accumulation rate at the outer radius could be smaller, thus reducing the rate of speed increase. The last curve is the Kepler speed curve only.

The galaxy may have started forming around $180[\mathrm{My}]$ and the main accumulation probably lasted nearly $1,2[\mathrm{~Gy}]$, far longer than any of the other galaxies described herein. For example, the calculated formation time for the MW is 320 [My], or 3,7 times shorter than UGC2885. The luminous mass of this galaxy is sufficient to generate its rotation speeds with the presence of the cosmological gravity. 


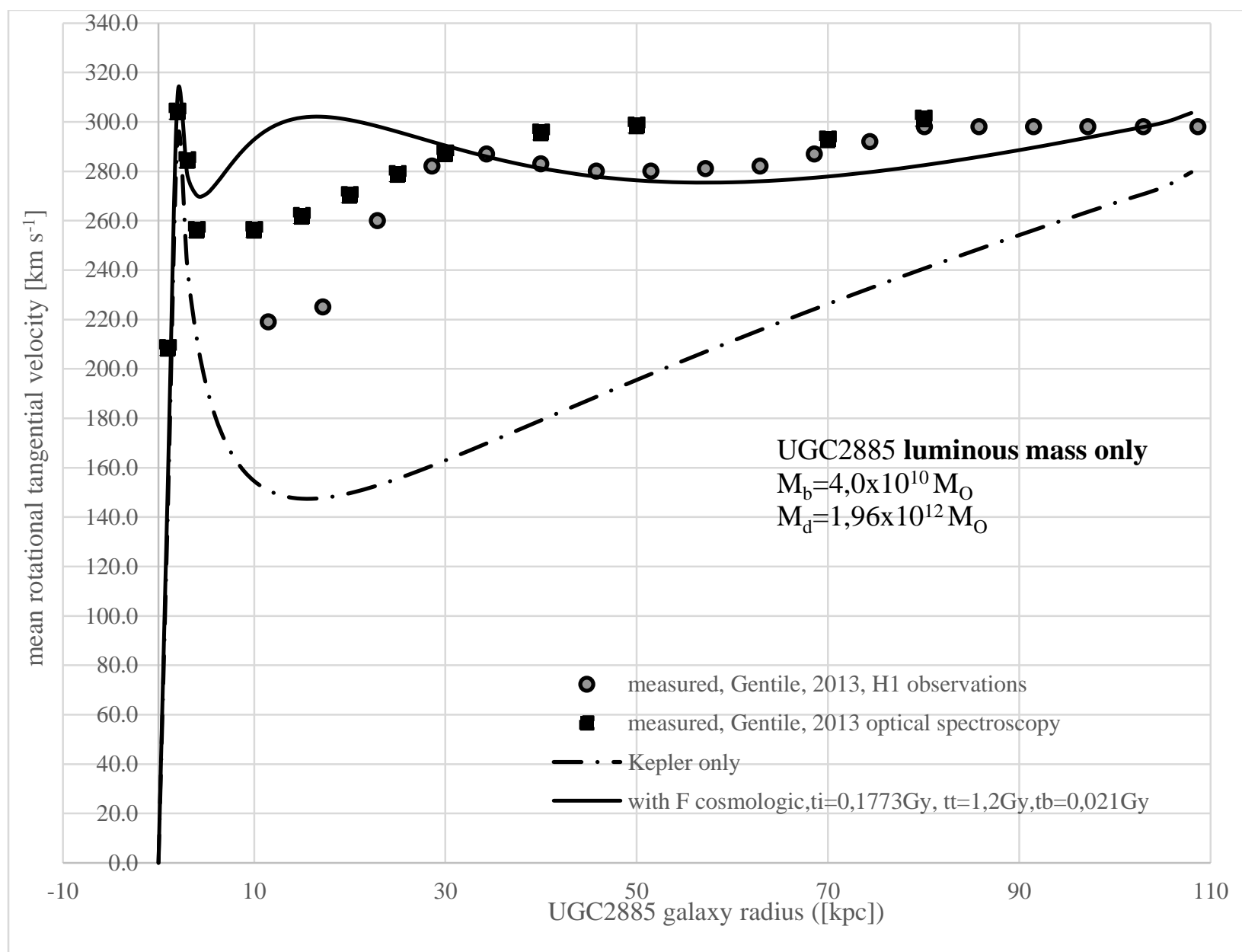

Figure 30: UGC2885 rotational velocities

\section{NGC253, Sculptor}

The rotation curve of this southern sky galaxy was measured by Pence (1981), with over 3,700 measurements made (Fabry-Perot) along the great axis of the galaxy. Figure 31 shows an approximation of the low and high values (range) derived from the means on groups of ten values. The profile becomes nearly flat beyond an estimated 2,25 [kpc] distance from the center. In fact, Pence suggests a mean rotation speed of $205 \mathrm{~km} \mathrm{~s}^{-1}$ for the measurement zone. He studied several rotation models as well as several estimated masses derived from these models (six estimated masses), varying between $1,08 \times 10^{11}$ and $1,54 \times 10^{11} \mathrm{M} \odot$, if the estimated mass of the galaxy is 
extrapolated beyond 7,9 [kpc], or the speed measurement zone. Indeed, the suggested rotation curve encompasses a radius of $\sim 15[\mathrm{kpc}]$ (20,5 arc-minutes). The total mass is an estimated $1,5 \times 10^{11} \mathrm{M}_{\odot}$, or twice that of the measurement zone (observed mass of 5-7 x $10^{10} \mathrm{M}_{\odot}$ for $\mathrm{r} \leq 7,9$ [kpc]). Center mass is an estimated $1,8 \times 10^{10} \mathrm{M}_{\odot}$. The velocity profile seems to correspond fairly well with measured values, with a tendency to increase. Adjustment of the velocity profile shows that this galaxy started to form before the MW, but that the center formation process took much longer to complete, or $42[\mathrm{My}]$, compared to $13[M y]$ for the MW. The mass of the MW center is a bit lower than NGC253, which is of larger estimated dimension however $(2,2$ [kpc] compared to $1[\mathrm{kpc}])$, which could partly explain the longer formation time of its center. The luminous mass of this galaxy is sufficient to genereate the observed rotation speeds.

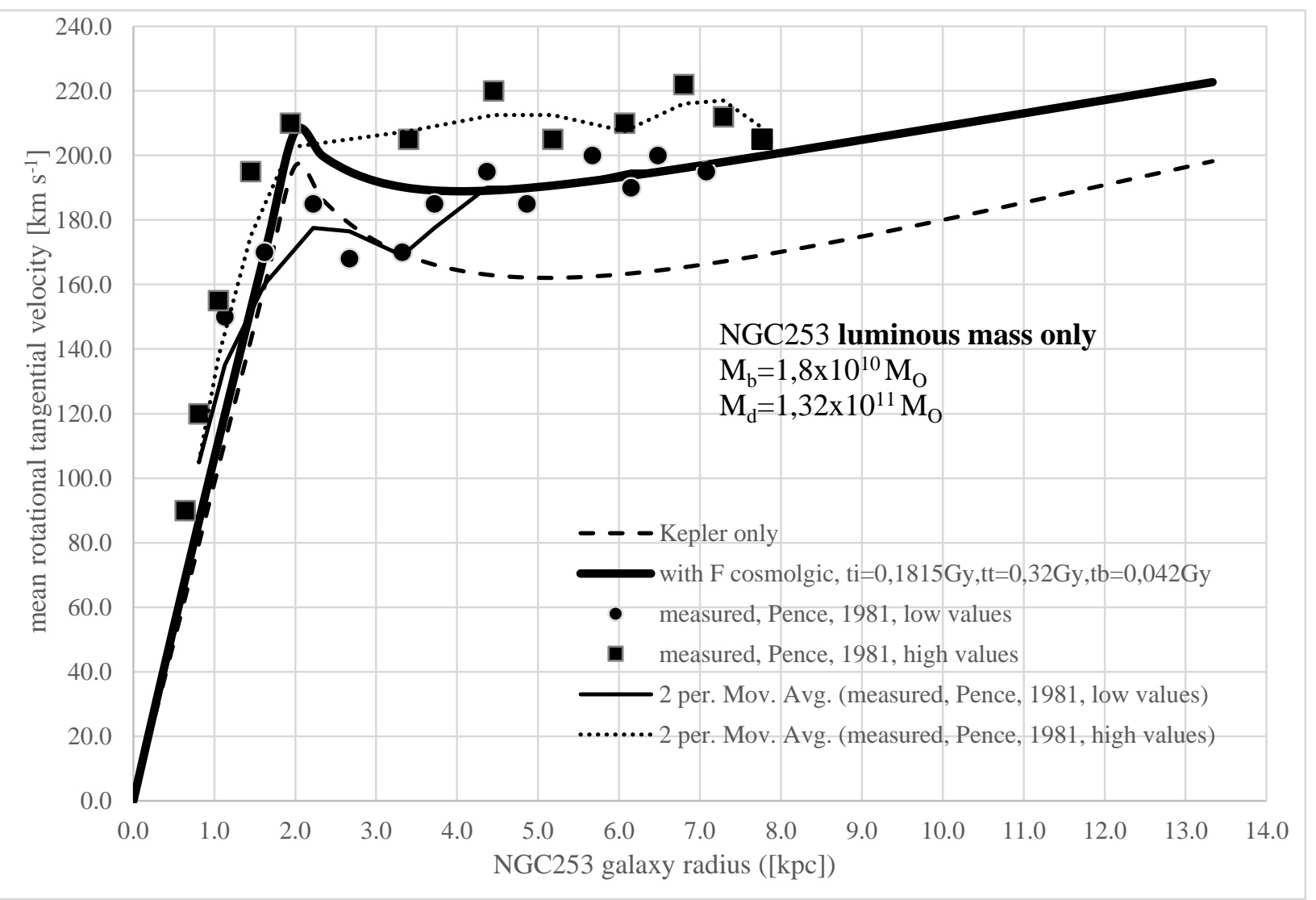

Figure 31: NGC253 rotational velocities 


\section{Irregular dwarf galaxy DDO161}

Dwarf galaxy DDO161 was chosen to show the later effects of cosmological gravity. Côté et al. (2000) studied eight irregular dwarf galaxies with the Australian telescope, and reported large amounts of dark matter. In the case of DDO161, they predicted a large ratio of dark matter vs. luminous matter $\mathrm{m}_{\text {dark }} / \mathrm{m}_{\text {lumi }} \sim 8$ to 9 to explain the observed rotation speeds. The observed luminous mass (stars and gases) is:

$$
M_{\text {total }}=M_{\text {star }+g a s} \sim 6,17 \times 10^{8} M_{\odot}
$$

In this case, we assume that the galaxy's mass is only the observable kind, which increases linearly from the center to the outer radius at $\sim 6,5[\mathrm{kpc}]\left(\mathrm{M}_{\mathrm{b}}=0\right)$. Figure 32 shows the observed and modeled rotation curves using cosmological gravity for a formation start time of about 220 [My] and total formation time of 240 [My]. The precision of the measurements made by Côte et al. (2000) is variable, but for this example we estimate a mean uncertainty of $\sim \pm 3\left[k m s^{-1}\right]$. This galaxy started to form after the MW ( $40[\mathrm{My}]$ later $)$, and formation lasted $\sim 240$ [My]. Its mean mass accumulation rate is much lower than that of the MW, or 2,57 [ $\left.\mathrm{M}_{\odot} y^{-1}\right]$ vs. $\sim 328\left[\mathrm{M}_{\odot} y^{-1}\right]$. Finally, dark matter does not have to be considered here to explain the rotation speeds of this dwarf galaxy. 


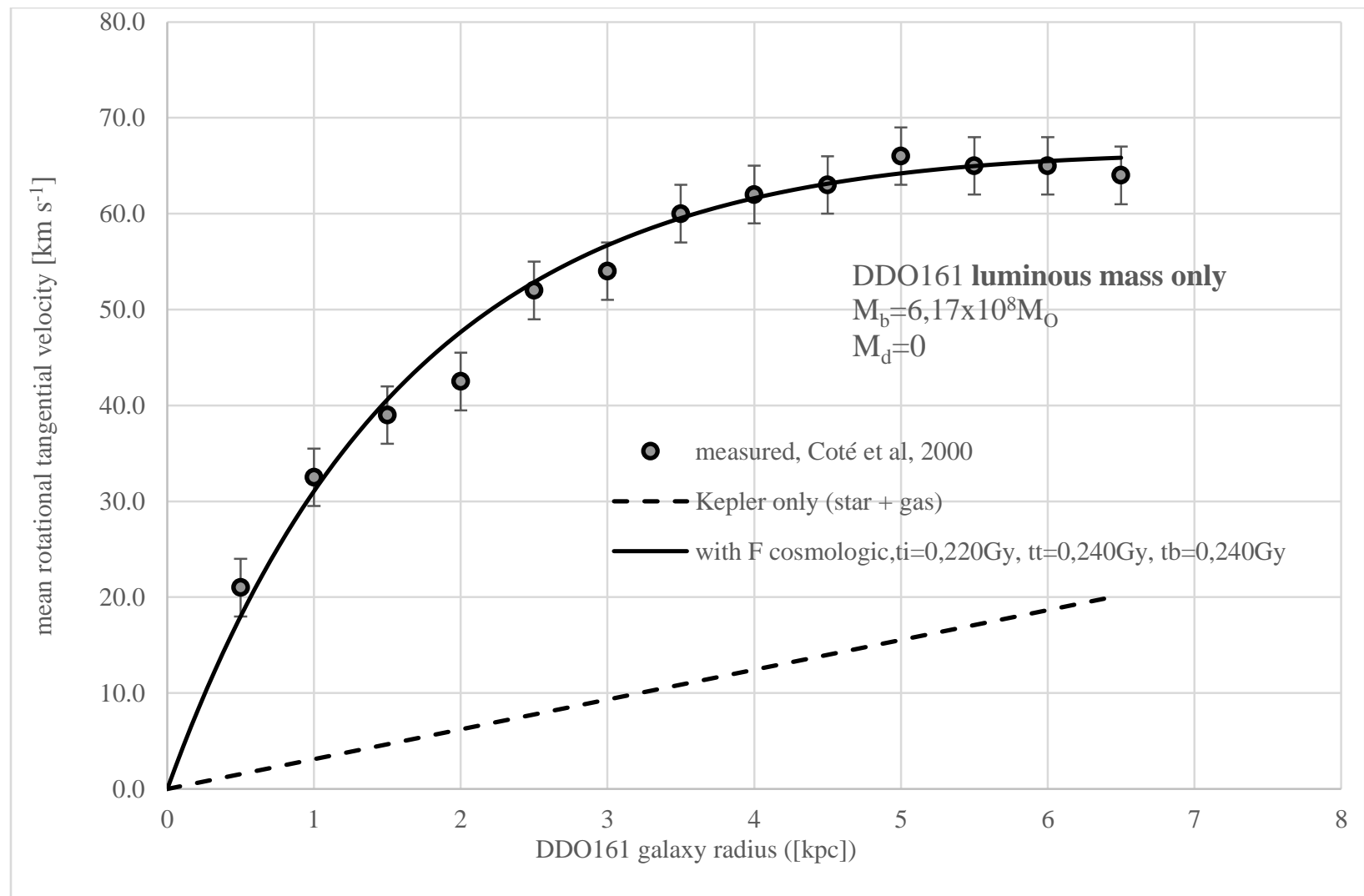

Figure 32: DDO161 rotational velocities

\section{UDG44, Dragonfly}

To demonstrate the powerful effects of the cosmological constant, UDG44, a diffuse galaxy in the Coma cluster, was studied by Van Dokkum et al. (2016), who concluded that dark matter makes up $98 \%$ of the galaxy's total mass $\left(1,9 \times 10^{10} \mathrm{M}_{\odot}\right)$, with an observable (luminous) mass of $3,8 \times 10^{8}$ $\mathrm{M}_{\odot}\left(\mathrm{r}_{1 / 2}=4,6[\mathrm{kpc}]\right)$. This galaxy is highly diffuse, although very massive according to researchers, and does not behave like the MW, being considered a "failed MW". Authors report that the galaxy's velocity profile is not structured, and that the mean speed is around $9\left[\mathrm{~km} \mathrm{~s}^{-1}\right]$ with large dispersion (unstructured), or $\sigma \sim 47\left[\mathrm{~km} \mathrm{~s}^{-1}\right]$. It is obvious that a Kepler rotation model would not apply here. However, to perceive the effects of cosmological gravity, the model can be used to see 
its effects during formation, which lasted between 1 and 5 [Gy], 3-15 times longer than the MW. Figure 33 shows the rotation speed relative to formation time, with a start time around 0,177 [Gy], around the same time as the MW.

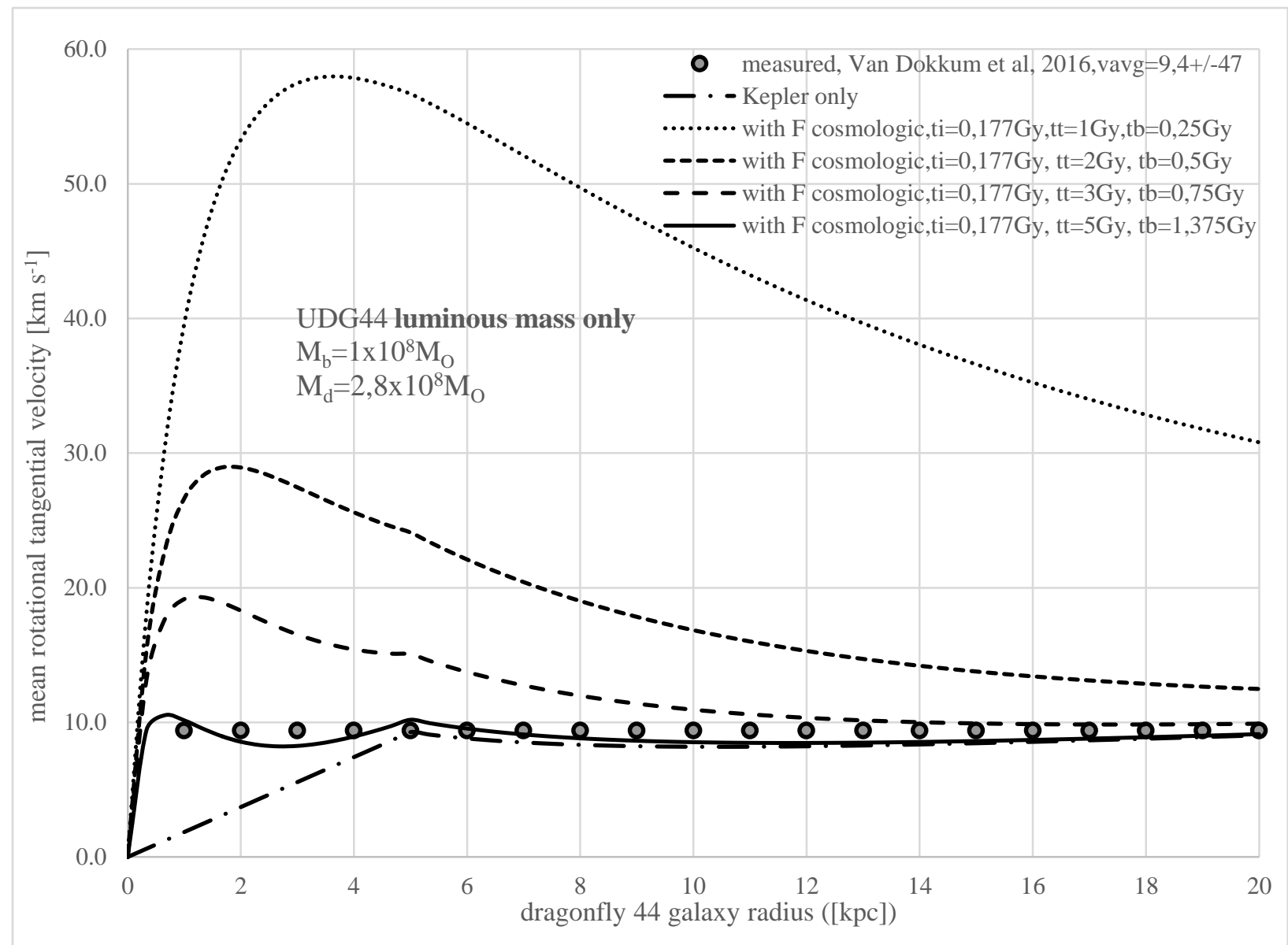

Figure 33: UDG44 rotational velocities

Note that the longer formation takes, the greater the decrease in cosmological gravity, meaning that rotation becomes almost Kepler-type, except for speeds in the smaller radii, which do not fit well with the Kepler model $(\mathrm{r}<5[\mathrm{kpc}])$. Note also the need to use cosmological gravity for the early formation of this galaxy - as a reminder, the observations made by Van Dokkum et al. (2016) showed no established rotation. Nevertheless, we know that when a galaxy takes a very long time 
to form, the effects of the cosmological gravity diminish and the velocity profile points to the Kepler model; but the Kepler model is not adequate for simulating rotation speeds for smaller radii, while cosmological gravity does so relatively well. In short, based on this model, for some unknown reason, but likely due to a lack of neighbouring matter, this galaxy seems to have taken a very long time to form. Knowing this, with the cosmological gravity getting smaller and smaller after $\sim 1[\mathrm{~Gy}]$, the galaxy never had the impetus to generate conventional rotation, making it a diffuse galaxy or, according to this model, a late-developing galaxy from the standpoint of mass accumulation. In fact, there are very many diffuse galaxies of this type in the Coma cluster $(>40)$, which tends to confirm the idea that the matter content in this area of space is rather poor, leading to the formation of diffuse galaxies.

\section{Galaxies cluster of Coma}

We have seen that the prediction model of the luminous mass rotation of a few galaxies, using the cosmological force of gravity, predicts fairly correctly the observed velocities. Of course, the nonluminous baryonic material exists but the quantities necessary to explain the rotational velocities are greatly diminished. Now it would be interesting to check whether on a larger scale (500 to 1000 times), this cosmological force of gravity can explain other mechanisms of rotation of matter. To do this, we apply the model of mass rotation at the scale of a galaxy cluster like that of the Coma cluster. Indeed, this cluster has been studied extensively since the 1930 s with among others the studies of Zwicki (1937) and thereafter those of Mayall (1960), Van Albada (1961), Omer et al. (1965), Pebbles (1970), Rood et al. (1972), Kent et al. (1982), Merrit (1987), White et al. (1993) and recently Gavazzi (2009). In summary, the various studies have all shown, to varying degrees, that the observed velocities of the $\sim 1000$ galaxies of the cluster can not be explained again by the presence of the luminous mass only estimated from the brightness-mass of galaxies ratio $\left(M_{\odot} / L_{\odot}\right)$. 
It was also with the study of this cluster that the concept of dark matter was proposed (Zwicki). The application of the rotation model is more complicated in the case of a galaxy cluster for mainly four reasons are:

- The clusters are much larger than the galaxies, with a rather spherical shape as well as a difficult boundary to determine precisely considering the surrounding objects.

- The clusters are remote and the Hubble-Lemaitre expansion effect is considerable (Hubble-Lemaître flow).

- Most galaxies and other objects in the cluster are not bright and more difficult to characterize.

- The velocities of the galaxies are observed from a line of sight that crosses the cluster in the direction of sight which causes a large variation of the observed velocities.

For the Coma cluster, for predicting the velocity of galaxies, we need to estimate the luminous mass $\mathrm{M}$, the time of the start of formation of the cluster $\mathrm{t}_{\mathrm{i}}$, the formation time of the cluster $\mathrm{t}_{\mathrm{T}}$ and the size of the cluster $r$. As an example, White et al. (1993), estimates the mass of stars and hot gas in the cluster from the observations of Godwin et al. (1977) To:

$$
\begin{gathered}
M_{\text {star }}=1 \pm 0,2 \times 10^{13} h_{70}^{-1} M_{\odot} \sim 1,43 \pm 0,3 \times 10^{13} M_{\odot} \\
M_{\text {gas }}=5,45 \pm 0,98 \times 10^{13} h_{70}^{-5 / 2} M_{\odot} \sim 13,3 \pm 2,4 \times 10^{13} M_{\odot} \\
M_{\text {star }+ \text { gas }}=1,47 \pm 0,27 \times 10^{14} M_{\odot}
\end{gathered}
$$

White estimates that the missing mass in the Coma cluster is about $90 \%$ of the total or $\sim 9$ times the luminous Mass. The following table 3 shows some values of the characteristics of the Coma cluster according to several authors and different databases. 
Table 3: Several authors of Coma cluster studies

reference radius radius, $\mathbf{r}$ (with $\mathrm{d}_{\odot} \sim 103 \mathrm{Mpc}$, $\mathrm{H}=73 \mathrm{~km} \mathrm{~s}^{-1} \mathrm{Mpc}^{-1}$ Nasa)

[은 $\quad[$ Mpc $]$

$\begin{array}{cc}\begin{array}{c}\text { Mean radial } \\ \text { velocity, } \\ <\bar{V}_{R}>\end{array} & \begin{array}{c}\text { Luminous } \\ \text { Mass } \\ M_{1}\end{array} \\ {\left[\text { km s }^{-1}\right]} & {\left[M_{\odot}\right]} \\ {[N \text { galaxies }]} & \end{array}$

Total Mass $\mathbf{M}_{\text {T }}$ (various models and $\mathbf{H}$ ) $\left[\boldsymbol{M}_{\odot}\right]$

\begin{tabular}{|c|c|c|c|c|c|}
\hline Zwicky (1937) & $\sim 2^{\circ} .25$ & $\begin{array}{l}\sim 13.8\left[H=55 \mathrm{kms}^{-1} \mathrm{Mpc}^{-1}\right] \\
\sim 10.4\left[H=73 \mathrm{kms}^{-1} \mathrm{Mpc}^{-1}\right]\end{array}$ & & - & $4,5 \times 10^{13}$ \\
\hline Mayall (1960) & $\sim 3^{\circ} .3$ & $\sim 5,9$ & $\begin{array}{c}\sim 6920 \\
47\end{array}$ & - & - \\
\hline $\begin{array}{l}\text { Van Albada } \\
(1961)\end{array}$ & $\sim 2^{\circ}$ & $\sim 3,6$ & - & - & - \\
\hline Abell (1965) & $\sim 2^{\circ} .5$ & $\sim 4,5$ & - & $6 \times 10^{12}$ & $2-5 \times 10^{14}$ \\
\hline $\begin{array}{l}\text { Omer Jr, Page et } \\
\text { al. (1965) }\end{array}$ & $\sim 1^{\circ} .65$ & $\sim 3$ & - & - & - \\
\hline Peebles (1970) & $\sim 2^{\circ} .5$ & $\sim 4,5$ & $\begin{array}{c}\sim 6925 \\
42\end{array}$ & - & $\begin{array}{c}\left(1,46 \times 10^{15} h_{50}^{-1} \mathrm{e}^{ \pm 0,3}\right) \\
2,1-3,9 \times 10^{15}\end{array}$ \\
\hline $\begin{array}{l}\text { Rood, Page et al. } \\
\qquad(1972)\end{array}$ & $\sim 3^{\circ} .7$ & $\sim 6,7$ & $\begin{array}{c}\sim 6888 \\
102\end{array}$ & $\begin{array}{c}\left(\sim \mathrm{M}_{\mathrm{t}} / 7\right) \\
3,88- \\
5,92 \times 10^{14}\end{array}$ & $2,72-4,15 \times 10^{15}$ \\
\hline $\begin{array}{l}\text { Chincarini and } \\
\text { Rood (1975) }\end{array}$ & $\sim 5^{\circ}$ & $\sim 9$ & $\begin{array}{c}0^{\circ}-1^{\circ} .67, \\
\sim 6946 \\
1^{\circ} .67-3^{\circ}, \\
\sim 7059 \\
3^{\circ}-5^{\circ}, \sim 6909\end{array}$ & - & - \\
\hline Abell (1977) & - & $\begin{array}{c}\sim 2,144 \times 10^{23} 8[\mathrm{~m}] \\
\sim 6,95\end{array}$ & $\begin{array}{l}\sim 6888 \\
\text { Rood }\end{array}$ & $\begin{array}{c}\left(\sim \mathrm{M}_{\mathrm{t}} / 3-\mathrm{M}_{\mathrm{t}} / 2\right) \\
1,6-10 \times 10^{14}\end{array}$ & $5-30 \times 10^{14}$ \\
\hline $\begin{array}{l}\text { Kent and Gunn } \\
\text { (1982) }\end{array}$ & $\begin{array}{l}\sim 4^{\circ} .7 \text { to } \\
6^{\circ} .8\end{array}$ & $\sim 8,5$ to 12,2 & - & - & $\begin{array}{c}\left(2,9 \times 10^{15} h_{50}^{-1}\right) \\
5,8 \times 10^{15}\end{array}$ \\
\hline Merritt (1987) & $\begin{array}{l}\sim 13^{\circ} \\
(\text { model })\end{array}$ & $\sim 23$ & $\begin{array}{l}\sim 6932 \\
296\end{array}$ & $\begin{array}{c}\left(\sim \mathrm{M}_{\mathrm{t}} / 3\right) \\
5,3-6,3 \times 10^{14}\end{array}$ & $\begin{array}{c}\left(1,6-1,9 \times 10^{15} h_{100}^{-1}\right) \\
1,6-1,9 \times 10^{15}\end{array}$ \\
\hline $\begin{array}{l}\text { White, Navarro } \\
\text { et al. (1993) }\end{array}$ & - & $\sim 6,95$ (Abell, 1977) & - & $1,47 \pm 0,3 \times 10^{14}$ & $1,21 \pm 0,26 \times 10^{15}$ \\
\hline $\begin{array}{l}\text { Gavazzi, Adami } \\
\text { et al. (2009) }\end{array}$ & - & - & - & - & $\begin{array}{c}\left(5,1_{-2,1}^{+4,3} \times 10^{14} h_{70}^{-1}\right) \\
0,42-1,34 \times 10^{15}\end{array}$ \\
\hline
\end{tabular}


We see that the observed and estimated characteristics are relatively variable between the authors. However, one observation is constant, i.e. the estimated observed (luminous) mass is less than 2 to 10 times compared to that estimated necessary to explain the observed rotational velocities of cluster galaxies. For the application of the model, several choices of values are possible. We arbitrarily select the values of Kent et al. (1982) which present a review of the authors data before them and the luminous mass estimated by White et al, i.e.:

$$
\begin{gathered}
r_{T} \sim 10,35[\mathrm{Mpc}] \\
M_{l} \sim 1,5 x 10^{14} M_{\odot}
\end{gathered}
$$

The following graphs show the results obtained for the Coma cluster. In order to allow for longer development and variable accretion in time of mass of a cluster compared to a galaxy, the expression of the growth of the radius $r$ (and mass) is modified slightly like this:

$$
r(t)=\alpha t^{b}=\frac{t^{b}}{t_{T}^{b}} r_{T}
$$

With $0<b<1$

For a circular rotation model, the tangential velocity is expressed as:

$$
v_{t}^{2}=G\left[\frac{\alpha^{2} t^{2 b}}{r_{b}^{3}} M_{b}+\frac{\left(\alpha^{2} t^{2 b}-r_{b}^{2}\right)}{\alpha t^{b}\left(r_{T}^{2}-r_{b}^{2}\right)} M_{d}\right]+\frac{k_{\Lambda} c^{2} \alpha^{2} t^{2 b}}{6\left(t_{i}+t\right)^{4}}
$$

We know that for a spherical geometry, mean quadratic radial velocity (line of sight) can be expressed from the quadratic velocity v, i.e.: 


$$
<\bar{V}_{R}>^{2}=3 v^{2}=3 v_{t}^{2}
$$

Three unknowns are to be determined, the time of the start of the formation of the cluster $t_{i}$, the duration of the formation of the cluster $\mathrm{t}_{\mathrm{T}}$ and the variable speed of progression of the formation of the cluster with exponant $b$. Several combinations are possible but we possess the measured values of the radial velocity of the galaxies according to the radius $\left\langle\bar{V}_{R}>\right.$ of the cluster as well as the standard deviations of the velocities $\sigma_{\mathrm{R}}$. The following 3 figures $34,35,36$ present the profile of the standard deviation $\sigma_{\mathrm{R}}$ for different plausible combinations of $\mathrm{t}_{\mathrm{i}}, \mathrm{t}_{\mathrm{T}}$ and $(\mathrm{b})$ and the measures taken by Kent (1982), Rood (1972) and Chincarini (1975). Several observations can be made:

- The standard deviations $\sigma_{\mathrm{R}}$ of $\left\langle\bar{V}_{R}>\right.$ are variable for small radii, calibration is more difficult for this area.

- $\quad$ The high values of $\mathrm{t}_{\mathrm{T}}(>3-4[G y])$ do not fit well with the values found for $\mathrm{r}>4000$ $[\mathrm{kpc}]$.

- The lower values of $\mathrm{t}_{\mathrm{i}}(<0.6[G y])$ do not fit well with the values found.

- The lower values of $\mathrm{b}(<0.5[G y])$ do not correspond well to the values found. 


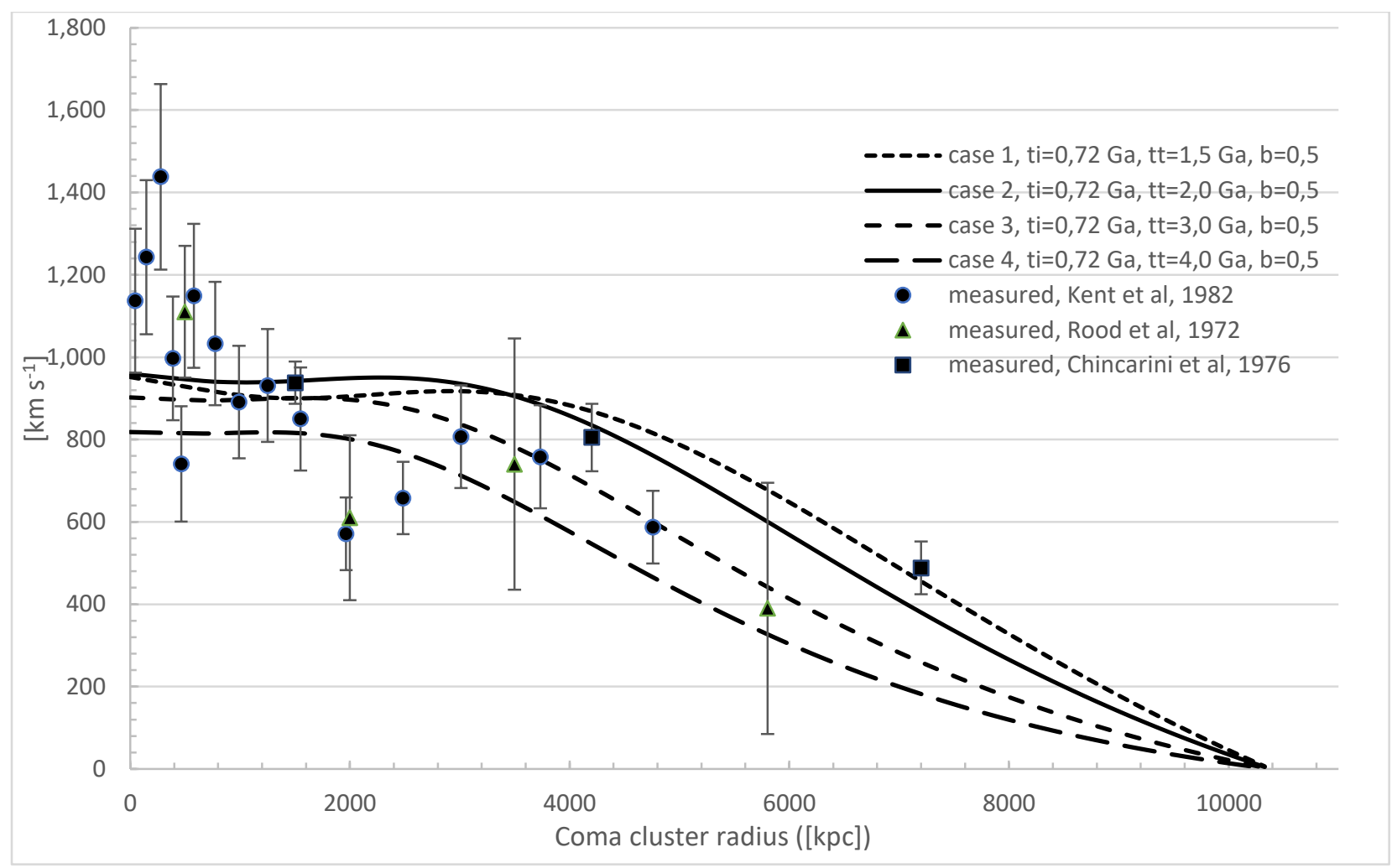

Figure 34: Coma cluster radial velocity dispersion profil

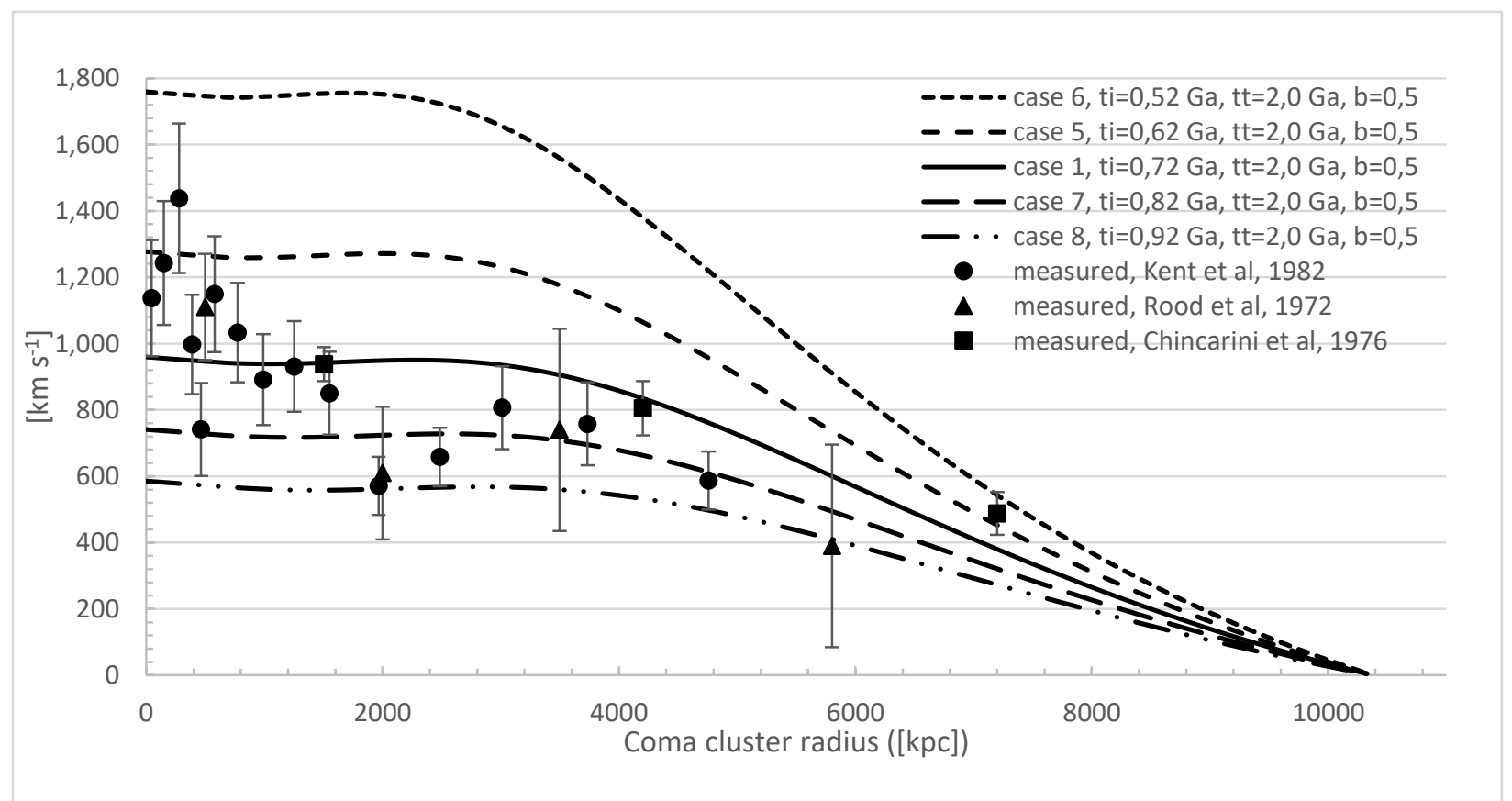

Figure 35: Coma cluster radial velocity dispersion profil 


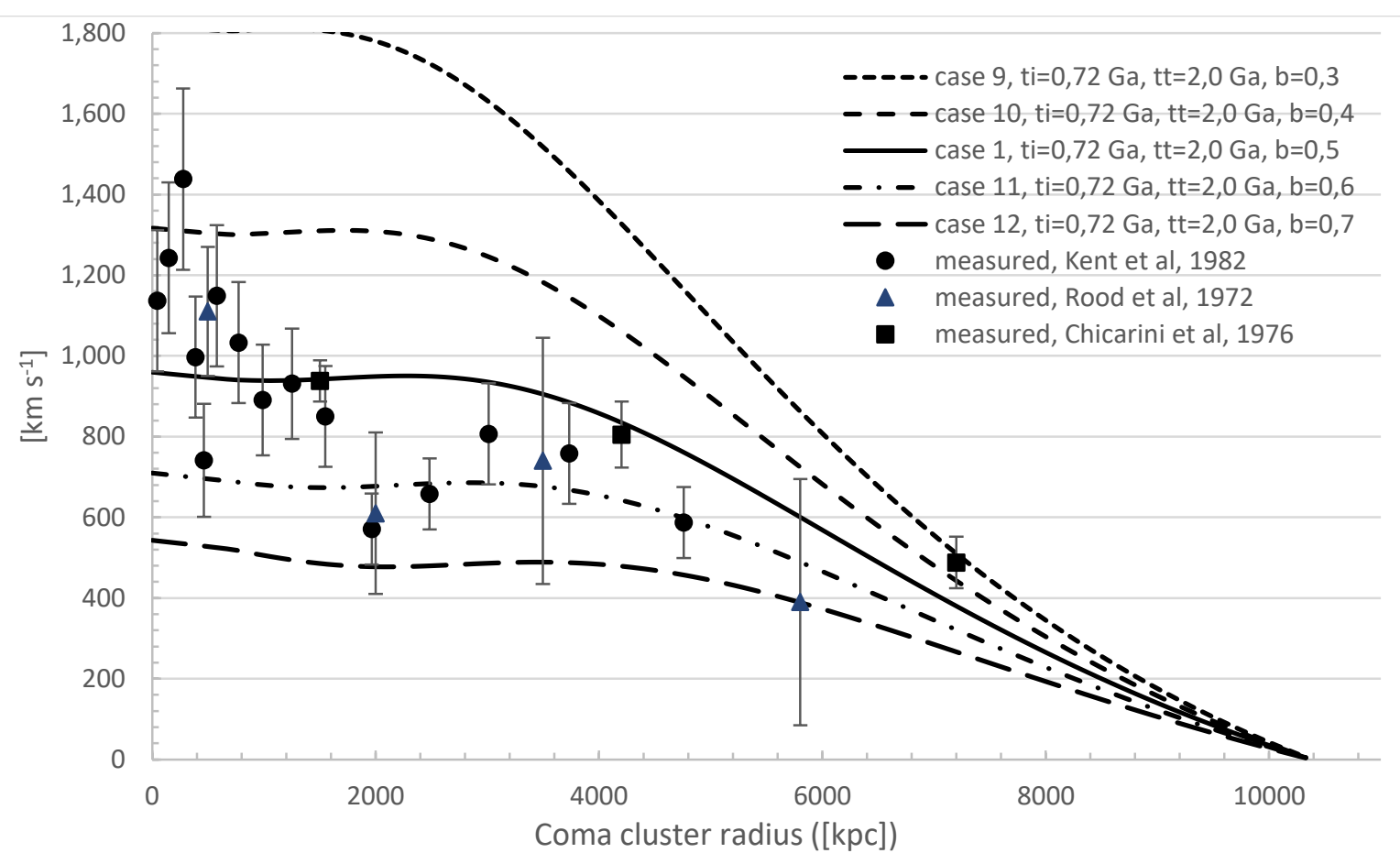

Figure 36: Coma cluster radial velocity dispersion profil

In summary, we observe that the beginning of the formation of the cluster is posterior to $\sim 0.6$ [Gy] and the duration of the formation is less than $\sim 2.5[G y]$. In addition, the growth rate of the cluster appears to be higher at the beginning $(b<1)$. If we choose the following preferred values $\left(t_{i}=\right.$ $0,7[G y], \mathrm{t}_{\mathrm{T}}=2,2[G y]$ and $\left.\mathrm{b}=0,5\right)$, we obtain the following velocity curves for the Coma cluster (Figure 37). 


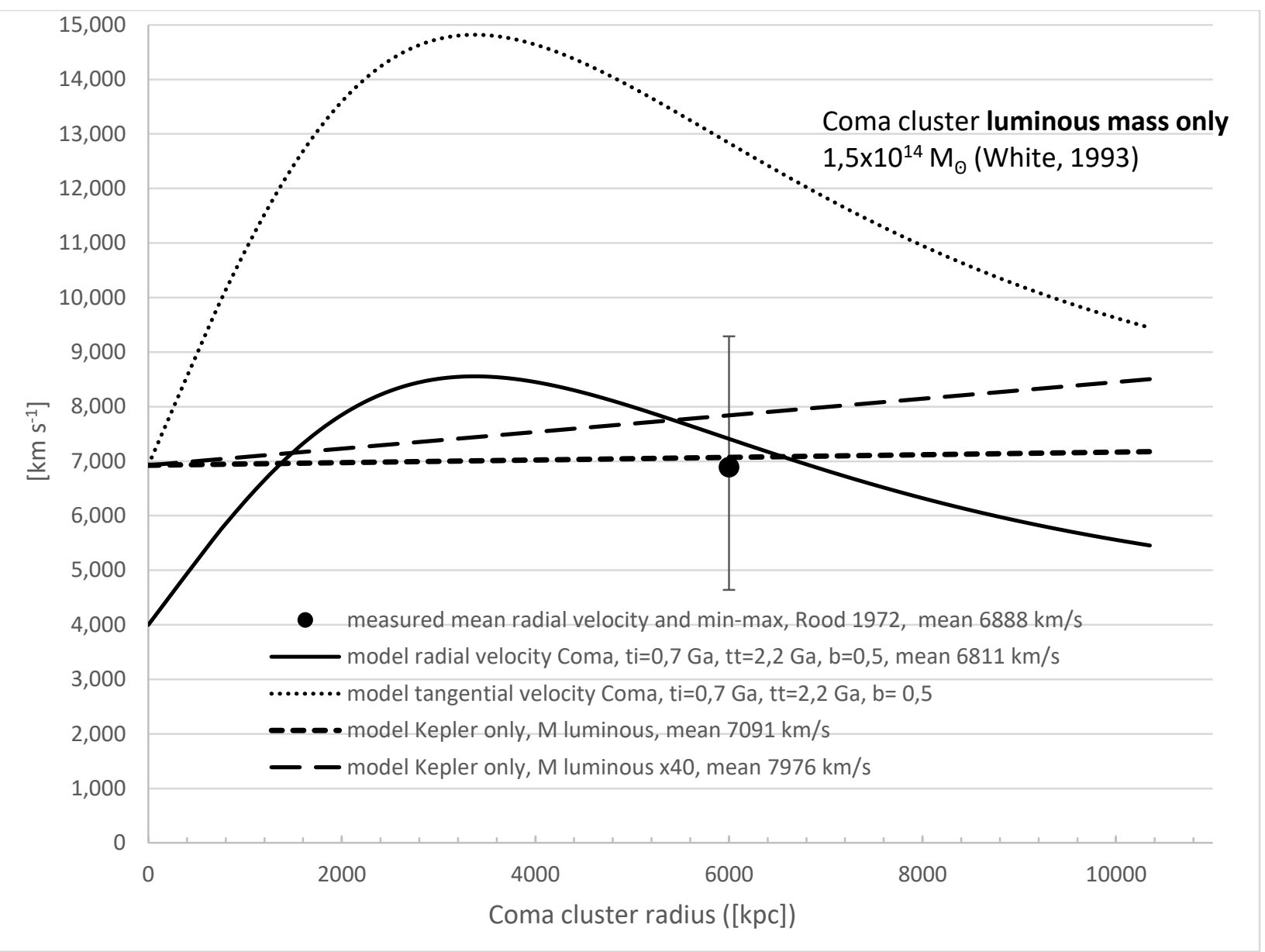

Figure 37: Coma cluster radial velocity dispersion profil

We can draw the following conclusions about the Coma cluster and the cosmological force $\mathrm{F}_{\Lambda}$.

1- Excluding this cosmological force, it is not possible to reproduce the observed radial velocities using only the luminous mass and gravitational force only. Indeed, the rotational velocities are too low. If the mass of the cluster is increased by 40 times, the observed elevated velocities can be obtained without the cosmological gravitational force.

2- The cosmological constant $\Lambda$ is to much decrease for a time of formation initial $t_{i}$ of 0.7 [Gy] compared to that of the MW of 0.18 [Gy] (228 times smaller) but, the $r$ dimension 
of the Coma cluster is $\sim 500$ times greater which allows to maintain the cosmological gravitational force in the case of a cluster of galaxies.

3- As an approximate comparison, the beginning of the formation of the Coma cluster is located $\sim 240[M y]$ later than the formation of a dwarf galaxy like DDO161 but $\sim 670$ $[M y]$ before the end of the formation of a giant galaxy such UGC2885. This suggests that the beginnings of the formation of clusters, similar to that of Coma, began when galaxies like the MW were already present but during the formation of the cluster which has duration $\sim 2.2[G y]$, the larger galaxies continued to develop and they have reached the maturity of their development before the end of the formation of the cluster.

4- If we look at Kent's (1982) database, we can find radial velocity values $<\bar{V}_{R}>$ as high as 13000-17000 $\left[\mathrm{km} \mathrm{s}^{-1}\right]$. This confirms that the values of the tangential velocity curve $\mathrm{vt}_{\mathrm{t}}$ In Figure 38 are realistic because the maximum observable speeds of $<\bar{V}_{R}>$ are precisely those of this tangential velocity when this one is practically aligned with the line of sight. However, without the cosmological gravitational force (Kepler only), it is not possible to obtain such large values of $\left\langle\bar{V}_{R}>\right.$ unless you increase the mass of the cluster by a 300x factor.

5- It is possible to estimate the evolution time of the UDG dragonfly 44 (Coma) compared to the time of formation of the Coma cluster. If we consider the angular separation of dragonfly 44 with the estimated center of the cluster, we find $\sim 1,035^{\circ}$ (Nasa Heasarc). This angular distance converted in length gives $\sim 1,86[M p c]$. This gives a time of evolution of dragonfly $44 \sim 2,2(1.86 / 10.35)$ [Gy] or $\sim 0,395$ [Gy] after the beginning of the cluster $(0,7[\mathrm{~Gy}])$ or a time of evolution of dragonfly 44 of $(1,09-0,177$ [Gy]) 0,91 [Gy] when joined to the cluster which is a lower value than the formation time of 
dragonfly 44 estimated at $\sim 5[\mathrm{~Gy}]$. This seems to indicate that the formation of galaxies is parallel to that of galaxy clusters, that is, the UDG galaxies as dragonfly 44 are probably not fully mature or formed when joined to the formation of a galaxy cluster.

In summary, with respect to the formation of a cluster of galaxies using the cosmological gravitational force, we observe that as in the case of galaxies, the formation appears faster than most estimates. However, lately, Tao Wang et al. (2016) observed a cluster called (protocluster) consisting of 17 massive galaxies and x-ray emission observations, suggests that the cluster formed rapidly from a dense nucleus of 80 [kpc] like a galaxy or even a black hole and the universe had only $2.5[G y]$. This suggests that the rapid formation of the clusters does indeed exist. Besides other clusters or protocluster seems to have been discovered according to the same authors.

\section{Summary of the galaxy rotation model}

We have seen that the five-parameter model performs relatively well for the simulation of mass velocity profiles for the seven galaxies and Coma cluster described above. The model shows mainly early formation of galaxies, which is not usually considered, although recent observations have shown that organized strucutures did exist as early as 400 [My]. However, the mass accumulation model (radius growth) is very basic, and a full-capacity accumulation model based on existing forces would be more realistic and would surely yield more accurate galactic growth rates. The model uses cosmological gravity, a major force during the initial billion years when the first galaxies were formed. Also, variation of the constant $G$ (as $G(r, t)$ ), used to adjust gravity forces for large radii, is not used in this mass rotation speed simulation model (Brownstein and Moffat, 2006). 
Observation of early galaxies is difficult due to their low brightness. Bouwens et al. (2006) reported that very few galaxies were formed before 700 [My]. However, some very old stars have been detected in the MW, which tends to confirm primitive formation of the galaxy before 700 [My]. For example, GN-Z11 is located 400 [My] after the beginning. If a fast formation time period is assumed, say 200 [My], this brings the start of formation to about 200 [My] after the beginning, which is in the order of magnitude of $t_{i}$ values yielded by this model. Galactogenesis is still a very wide-open question and delaying the start times of galaxy formation can be done by changing the accumulation rates or increasing the age of the universe, because cosmological gravity depends on the cosmological constant, which is cosmic time dependent, $t_{\Omega}$. With cosmological gravity in play, there is less of a need to turn to lacking or unobserved mass (like dark matter - existing but nonluminous baryonic matter) to explain the rotation patterns of many galaxies. In brief, from the standpoint of observable and unobservable masses of these eight galaxies, we had recourse to dark matter (non-luminous) for two of the galaxies (M33 and NGC3198). As for the other galaxies, only the observable luminous mass and cosmological gravity were used.

What is interesting with this model is the fact that it was derived from the analysis of a model of the universe that estimates the evolution of energy to calculate new dynamic parameters, such as the cosmological constant and cosmological gravity. Moreover, the mass rotation model for galaxies provides estimated formation times from early formation, $t_{i}$, as well as formation time, $t_{t}$, by adjusting these two parameters with the observed rotation curves and observed masses. In our opinion, no other model uses these dynamic parameters for the formation of galaxies with the cosmological constant. Finally, the mass accumulation model described herein is a very simple one; nevertheless, the results of rotation speed simulations are promising. Of course, a much greater number of galaxies should be studied with this rotation model to further improve and 
develop its potential. However, we can generate a graph (Fig. 38) showing the relation between total observed mass and formation times of these galaxies, with the exception of UDG44, which does not fit the model due to a significant amount of time of formation. The graph shows that formation time increases with total mass, which is quite plausible.

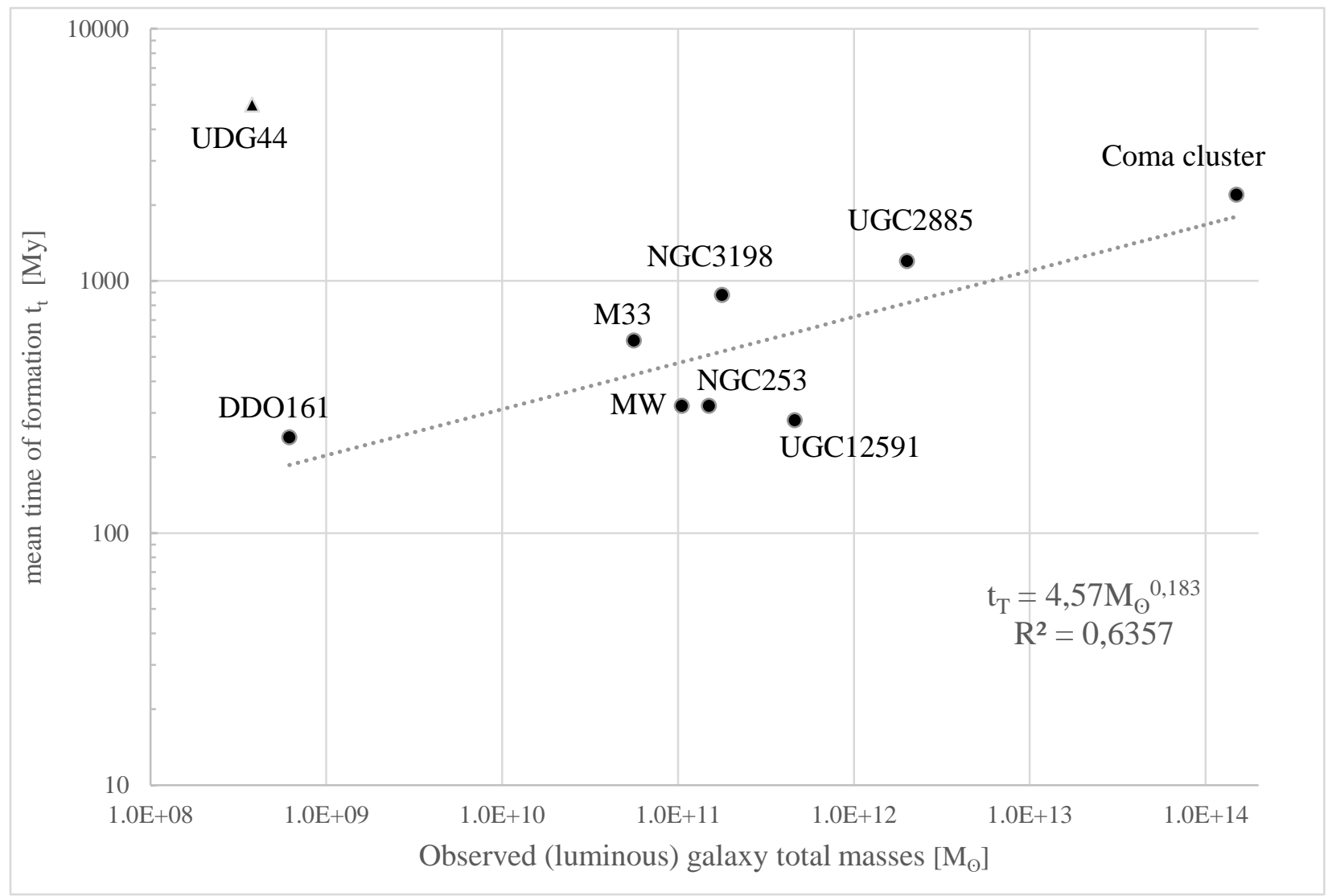

Figure 38: Galaxies tendency of main formation time fonction of luminous masses

\section{Relative position of galaxies}

While this may seem unlikely, using the matter expansion equation and estimated initial time of formation of a galaxy center $\left(\mathrm{t}_{\mathrm{i}}\right)$, we can derive an approximate cosmic time location of that galaxy relative to the MW. For example, using the estimated times to adjust rotation, we found that the formation of UGC12591 galactic center was completed around 176 [My] after the beginning (see 
table 4 below). The estimated distance of this galaxy is $\sim 394+/-133$ [My], or a maximum \pm 527 [My], which corresponds with an age limit for the formation of the center in the range of 13,27 $[\mathrm{Gy}] \leq \mathrm{t} \leq 14,33[\mathrm{~Gy}]$. With a scale factor of an initial sphere of $1[\mathrm{~Gy}]\left(\mathrm{t}_{\Omega} / \mathrm{t}=76,1[\mathrm{~Gy}] / 1[\mathrm{~Gy}]\right)$, we find that the center of this galaxy was probably formed between 174 [My] and 188 [My] $(13,27[\mathrm{~Gy}] / 76,1[\mathrm{~Gy}]$ and 14,33[Gy]/76,1[Gy]) after the beginning. Comparing the above values with the rotation curve (176 [My]), we find that this galaxy started to form around 5 [My] before the MW. Of course, these data are the result of manual adjustments of parameters $m_{b}, m_{d}, t_{i}, t_{b}$ et $t_{T}$, which leaves a fairly large margin of error. However, if the notion of this model for an approximate time of formation of the bulbe of these galaxies is accepted and we also accept that there is a characteristic or preferable time to the formation of galaxies, this could open a way to determine a preferred direction towards the beginning. This idea of a definite direction was addressed by Zhou et al. (2017). Indeed, from the study of observed acceleration variations, gobs, they determined two precise but diametrically opposed galactic directions $(1, \mathrm{~b})$ and $\left(1+180^{\circ}\right.$,b), where the accelerations of 147 galaxies show systematic differences that lead to two most likely directions. They used the MOND theory to derive these directions, along with values for $\mathrm{a}_{0}$ which, as we will see later, are fundamentally related to the cosmological constant, which depends on cosmic time, and to the formation time of the structure. Therefore, a more methodical study of the rotation of many galaxies around the galactic sphere would help to determine, with rotation curves and estimated masses, if a formation trend before or after the MW could yield a specific direction, and thus confirm or reject the notion of a possible direction towards the beginning. 
Table 4: Beginning of formation time of some galaxies

FROM OBSERVATION

\begin{tabular}{|c|c|c|c|c|c|c|}
\hline Galaxy & $\begin{array}{c}\text { de } \\
(\text { now }) \\
{[M y]}\end{array}$ & $\begin{array}{c}\boldsymbol{t}_{/} \boldsymbol{t}_{\Omega} \\
(\min \text { and max) } \\
{[-]}\end{array}$ & $\begin{array}{c}\boldsymbol{t}_{\boldsymbol{i}} \\
(\min \text { and } \mathbf{m a x}) \\
{[G y]}\end{array}$ & {$[G y]$} & {$[G y]$} & $\left(\boldsymbol{t}_{i}\right)^{m w}-\left(\boldsymbol{t}_{i}\right)$ \\
\hline MW & 0 & $13,8 / 76,1$ & 0,1814 & 0,1814 & 0,013 & 0 \\
\hline UGC12591 & $394 \pm 133$ & $13,27 / 76,1-14,33 / 76,1$ & $0,1744-0,1882$ & 0,1764 & 0,056 & $\sim 5,0$ \\
\hline UGC2885 & 310 & $13,49 / 76,1-14,11 / 76,1$ & $0,1772-0,1854$ & 0,1773 & 0,021 & $\sim 4,1$ \\
\hline NGC3198 & 47 & $13,75 / 76,1-13,85 / 76,1$ & $0,1807-0,1819$ & 0,1808 & 0,039 & $\sim 0,61$ \\
\hline M33 & 2,38 à 3,07 & $13,797 / 76,1-13,803 / 76,1$ & $0,1813-0,1814$ & 0,1814 & 0,058 & $\sim 0,03$ \\
\hline UDG44 & 330 & $13,47 / 76,1-14,13 / 76,1$ & $0,1770-0,1856$ & $\sim 0,1771$ & $\sim 1,0$ & $\sim 4,3$ \\
\hline $\mathrm{NGC} 253$ & 10,8 & $13,69 / 76.1-13,91 / 76,1$ & $0,1812-0,1815$ & 0,1815 & 0,042 & $\sim-0,1$ \\
\hline
\end{tabular}

\section{MOND theory and cosmological constant}

The cosmological constant can be used to find a possible fundamental explanation for the MOND theory. Indeed, by equalizing the expression of rotation speed for the mass of a great structure, as predicted with the MOND theory, to that obtained using conventional and cosmological gravity, we get the following equation of equality:

$$
\frac{v^{2}}{r}=a_{M O N D}=a_{G+\Lambda}
$$

Or

$$
v_{M O N D}=\sqrt[4]{G M a_{0}}=\sqrt[2]{\frac{G M}{r}+\frac{\Lambda c^{2} r^{2}}{6}}
$$


From this expression, a more fundamental expression is obtained for the constant $a_{0}$ of this theory $\left(a_{0} \sim 1,2 \times 10^{-10}\left[\mathrm{~ms}^{-2}\right]\right)$, after a few manipulations, we get:

$$
a_{0}=\frac{G M}{r^{2}}+\frac{\Lambda c^{2} r}{3}+\frac{\Lambda^{2} c^{4} r^{4}}{36 G M}
$$

Or still, with the mean density of the structure:

$$
a_{0}=\frac{4 \pi G \rho r}{3}+\frac{\Lambda c^{2} r}{3}+\frac{\Lambda^{2} c^{4} r}{48 \pi G \rho}=\frac{r}{3}\left(4 \pi G \rho+\Lambda c^{2}+\frac{\Lambda^{2} c^{4}}{16 \pi G \rho}\right)
$$

First, constant $a_{0}$ is not independent of time. Indeed, it varies with the age of the universe via the cosmological constant, radius, and mass of the structure. Hence, when the value for $\mathrm{a}_{0}$ is adjusted, or selected, those three parameters are fixed. However, we know that the value of $\Lambda$ is time dependent, so that the choice of $r$ and $M$, in particular, fix the value of $\Lambda$, or the mean formation time of the structure. Selecting a typical mass and typical radius for a galaxy is easy (e.g. $10^{10} \mathrm{M}_{\odot}$ and $\mathrm{r}=40[\mathrm{kpc}])$. For smaller structures, $\mathrm{r} \rightarrow 0$, the last two terms tend towards zero, which brings us back to Newton's theory:

$$
\begin{gathered}
\frac{a^{2}}{a_{0}}=\frac{G M}{r^{2}} \\
a=\sqrt{a_{0} \frac{G M}{r^{2}}}=\frac{G M}{r^{2}} \text { and } v=\sqrt{\frac{G M}{r}}
\end{gathered}
$$

If $\mathrm{r}$ increases, the final term becomes dominant, or:

$$
a_{0} \rightarrow \frac{\Lambda^{2} c^{4} r^{4}}{36 G M}=\frac{\Lambda^{2} c^{4} r}{48 \pi G \rho} \rightarrow 3,36 \times 10^{42} \Lambda^{2} \frac{r^{4}}{M}
$$

Whith 


$$
a=\frac{\Lambda c^{2} r}{6} \text { and } v=\frac{\sqrt{\Lambda}}{\sqrt{6}} c r
$$

By selecting a typical mass and radius, a specific value for $a_{0}$ through time can be obtained, knowing that the cosmological constant will vary. Randriamampandry et al. (Randriamampandry and Carignan, 2014) use the MOND theory for the study of the rotation of 15 galaxies and they mention the need to vary the constant $\mathrm{a}_{0}$ in order to adjust the rotation curves ( $\mathrm{a}_{0} \sim 0.34$ to $2 \times 10^{-10}$ $\left.\left[\mathrm{ms}^{-2}\right]\right)$. The figure 39 shows the values of $a_{0}$ for three typical masses, $\mathrm{M}\left(10^{9}, 10^{10}\right.$ and $10^{11}$ $\left.\mathrm{M}_{\odot}\right)$, and radii, $\mathrm{r}(20$ and $40[\mathrm{kpc}])$.

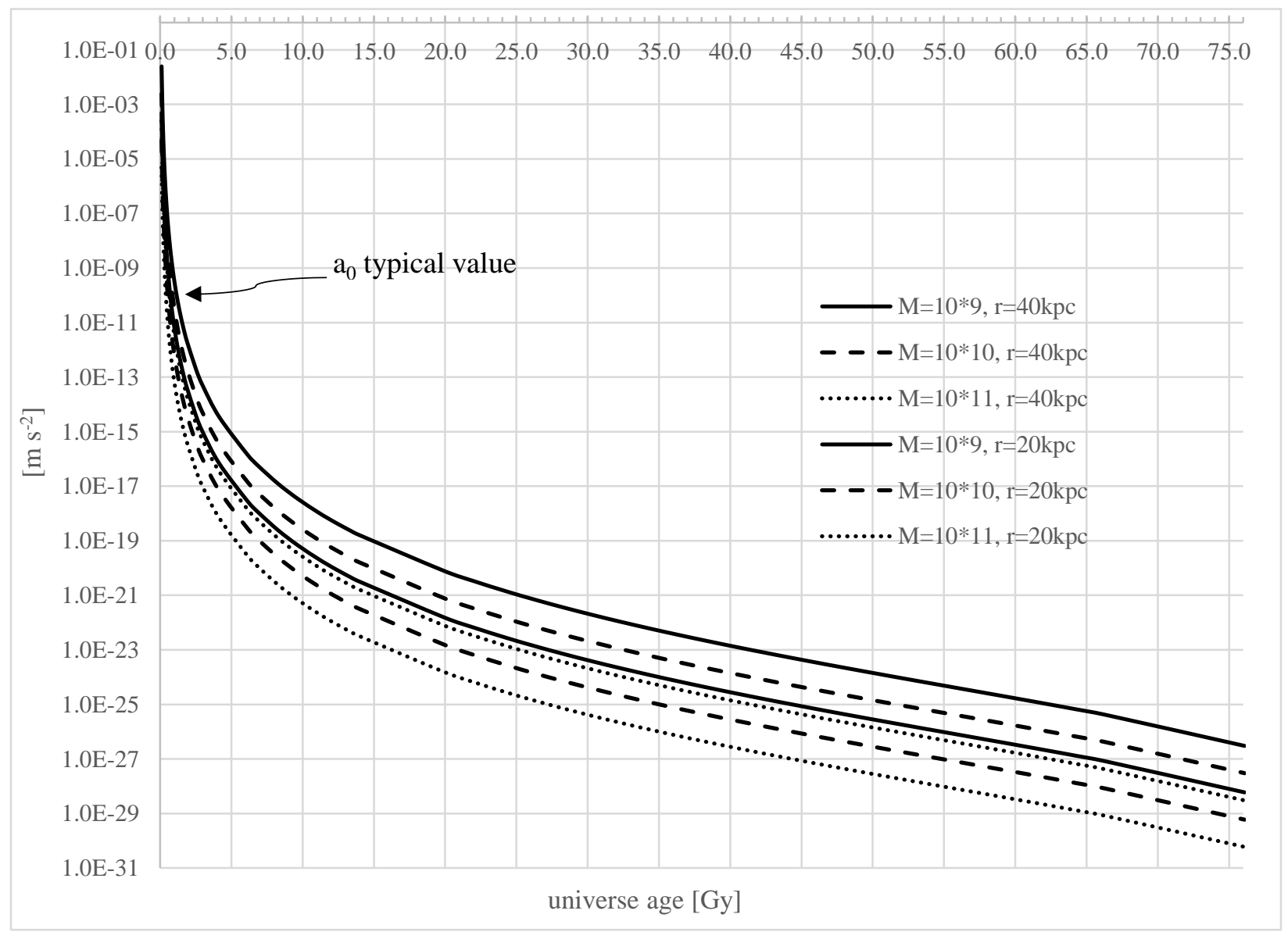

Figure 39: MOND, variation of constant $\mathrm{a}_{0}$ with time or $\Lambda(\mathrm{r}=20$ and $40[\mathrm{kpc}])$ 
Note that the selected value for $a_{0}\left(\sim 1,2 \times 10^{-10}\left[\mathrm{~ms}^{-2}\right]\right)$ corresponds to the formation periods of structures from about 0,32 [Gy] to 1 [Gy] (Fig. 40), showing that the MOND theory, in the context of this model, assumes that the galaxies were formed during that $\sim 600$ [My] period, so a short period of time also, but later than advanced in this model, in which formation starts around 200 $[M y](\mathrm{MW})$.

However, Lake (1989) and Schuberth (2006) used lower and higher values for $\mathrm{a}_{0}\left(2,5 \times 10^{-11}\left[\mathrm{~ms}^{-2}\right]\right.$, $\left.1,2 \times 10^{-8}\left[\mathrm{~ms}^{-2}\right]\right)$ in an effort to explain the rotations of six galaxies (Lake) and one globular cluster NGC4636 (Schuberth), which could mean earlier or later formation times, as early as 200 [My] and as late as 450 [My] (Fig 40). Finally, we can see that the MOND theory provides very good models of galaxy rotation, as it relies on the constant $\mathrm{a}_{0}$, which in turn relies on the cosmological constant, $\Lambda$, which represents the cosmological gravity, $F_{\Lambda}$, required to explain the rotation speeds of galaxies. However, the MOND theory does not question the value of G or Newton's gravity theory. Indeed, for a cosmological constant value of zero, the Newtonian case is obtained, regardless of mass and radius. 


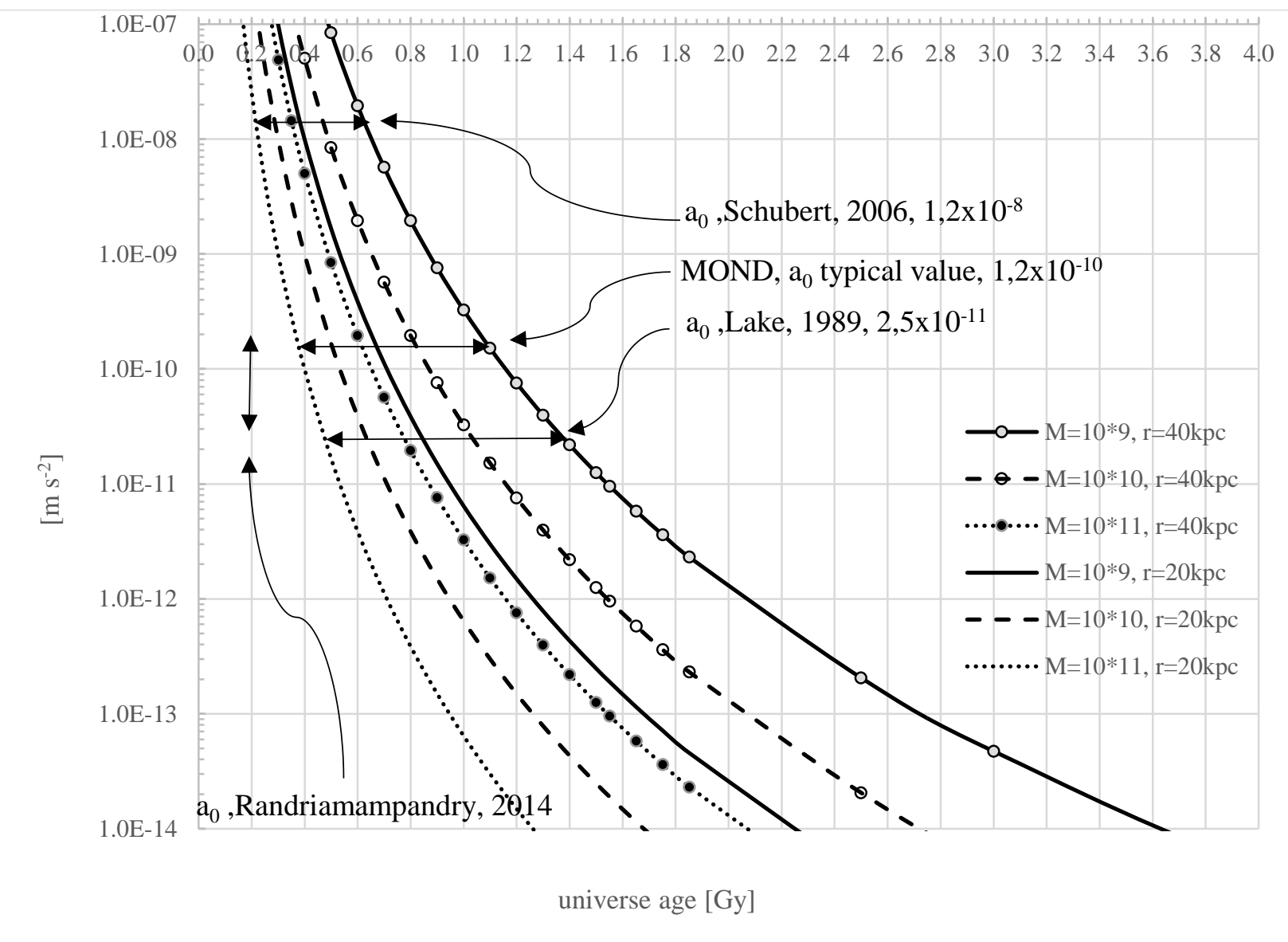

Figure 40: Variation of constant $\mathrm{a}_{0}$, MOND with time or $\Lambda\left(\mathrm{r}=20\right.$ and $40[\mathrm{kpc}]$ and $\mathrm{M}=10^{9}$ to $\left.10^{11} \mathrm{M}_{\odot}\right)$

\section{Conclusion}

The model proposed herein sheds light on the importance of the cosmological constant, $\Lambda$, which acts as a dominant gravitational force in the early universe. The model does not consider the existence of energy other than photons. In other words, the notion of dark energy, dark matter (non-baryonic) is not specifically addressed in the model, although the existence of some baryonic dark matter is accepted. The model provides a possible solution to the horizon problem with the 
concordance of photon volume with universe volume, the causality recovery period after $z=10^{26}$ and last scattering surface $\mathrm{z}=1098$. The model questions certain elements of the cosmological principle, that is the idea that there is no preferred position. The model assumes that the MW occupies a precise location (cosmic time 13,8 [Gy]), and not a central one in this universe of possible $\sim 76$ [Gy] cosmic age. Moreover, we do not have sufficient data from cosmological observations to claim with assurance that the universe is the same in all directions and, more specifically, to the high values of $z$, excluding the $\mathrm{CMB}$, which appears in the early universe before the formation of structure that we observe, which in turn is subject to a different chronology. Indeed, the observed percentage of this universe is extremely low, especially as concerns galaxies. If the number of galaxies is an estimated $\sim 2 \times 10^{12}$, less than $\sim 10^{-6}$ percent have been indexed $(90,000$ galaxies) (Vipers, 2016). The model can partially describe the rotation of certain galaxies without recourse to dark matter (halo), but rather uses the cosmological gravity effect, which has a heavy impact during the early formation period. The galaxies studied herein appear very early in the model, or within the first billion years. In fact, recent observations (Aduado et al, 2018) show the possibility that a star, J0815+4729, had already formed in the MW as early as 200 [My]. Moreover, recent observations of dwarf galaxy MACS1149-JD1 and its star population show that the galaxy already existed at $\sim 500$ [My], and that it had already started to form as early as $\sim 160-200$ [My] after the beginning (Hoag, Bradač, Brammer et al., 2018); (Hashimoto, Laporte et al., 2018). Another galaxy detected at $\mathrm{z}=6,027$ already has a population of stars aged $800[M y](\mathrm{z} \sim 18)$ or $\sim 200[M y]$ after the beginning, Richard et al. (2011). Cosmological gravity is behind such early formation, prior to the accepted normal period of a few billion years. Of course, this does not exclude the relative activity of galaxies thereafter (accumulations, collisions, amalgamations, breakups). The baryonic mass of the universe could be as much as $~ 500$ times smaller than 
formerly estimated and accepted. However, it should be noted that no one has been able to estimate that mass without the use of predictive models, meaning that such mass could be in fact lower than normally accepted values. Finally, in the context of this model, which uses the cosmological constant, the value of constant $\mathrm{a}_{0}$ of the MOND theory is more fundamentally explained, allowing to highlight the fact that the theory is an explicit form of cosmological gravity acting on the formation of galaxies. Constant $\mathrm{a}_{0}$ is not fundamentally a constant, and it does not question Newton's law of gravity for great structures. Finally, the model described herein seems interesting for several reasons, but further development is required before its foundations can be validated (complete particle generation, atoms, fusion etc.). The model is still one among many, fine tuning and improvements are to be expected.

\section{Conflicts of Interest}

The author declares that there is no conflict of interest regarding the publication of this paper.

\section{Funding Statement}

Funding for this article was supported by the University of Quebec at Chicoutimi

\section{Acknowledgement}

The author would like to thank the members of his family, especially his spouse (Danielle) who with patience to bear this work as well his childrens (Pierre-Luc, Vincent, Claudia), for their encouragement to persevere despite the more difficult periods. Also, a big thank you to Mrs. Nadia Villeneuve of UQAC who has prepared the article and references in an acceptable version. Finally, thanks to the University of Quebec at Chicoutimi and to the colleagues of the Department of Applied Sciences for their supports in the realization of this work. 


\section{References}

Abell, G.O., 1965, Annual Review of Astronomy and Astrophysics 3(1): 1.

Abell, G.O., 1977, The Astrophysical Journal 213: 327.

Amendola, L., et al., 2018, Cosmology and fundamental physics with the Euclid satellite, Springer.

Beneš, P., Faessler, A., Kovalenko, S., \& Šimkovic, F., 2005, Physical Review D 71(7): 077901.

Bernreuther, W., 2002, Lect. Notes Phys. 591: 237.

Bezrukov, F., Chudaykin, A., \& Gorbunov, D., 2018, Submitted.

Bressi, G., Carugno, G., Onofrio, R., \& Ruoso, G., 2002, Physical Review Letters 88(4): 041804.

Brownstein, J.R., \& Moffat, J.W., 2006, The Astrophysical Journal 636(2): 721.

Carmeli, M., \& Kuzmenko, T., 2001, AIP Conference Proceedings 586(1): 316.

Carvalho, J.C., 1995, International Journal of Theoretical Physics 34(12): 2507.

Cercignani, C., \& Medeiros Kremer, G., 2002, Relativistic Boltzmann Equation. in The Relativistic Boltzmann Equation: Theory and Applications, ed C. Cercignani, \& G. Medeiros Kremer (Birkhäuser Basel, Switzerland, 31).

Chincarini, G., \& Rood, H.J., 1975, Nature 257(5524): 294.

Conseil Européen pour la Recherche Nucléaire, 2018, Du vide à l'ultravide - le CERN fait le plein de technologies.

Corbelli, E., 2003, Monthly Notices of the Royal Astronomical Society 342(1): 199.

Côté, S., Carignan, C., \& Freeman, K.C., 2000, The Astronomical Journal 120(6): 3027.

Decca, R.S., López, D., Fischbach, E., et al., 2007, Physical Review D 75(7): 077101.

Dolgov, A.D., 1998, Surveys in High Energy Physics 13(1-3): 83.

Eisenstein, D.J., Zehavi, I., Hogg, D.W., et al., 2005, The Astrophysical Journal 633(2): 560.

Farnes, J.S., 2018, A\&A 620: A92.

Fixsen, D.J., 2009, The Astrophysical Journal 707(2): 916.

Frebel, A., Christlieb, N., Norris, J.E., et al., 2008, The Astrophysical Journal Draft.

Gavazzi, R., Adami, C., Durret, F., et al., 2009, Astronomy \& Astrophysics 498(2): L33.

Gentile, G., Józsa, G.I.G., Serra, P., et al., 2013, Astronomy \& Astrophysics 554: A125.

Gessner, E., 1992, Astrophysics and Space Science 194(2): 197.

Giovanelli, R., Haynes, M.P., Rubin, V.C., \& Ford, W.K., Jr, 1986, The Astrophysical Journal 301: L7.

Godwin, J.G., \& Peach, J.V., 1977, Monthly Notices of the Royal Astronomical Society 181(2): 323.

Guth, A.H., \& Steinhardt, P.J., 1984, Scientific American 250(5): 116.

Hashimoto, T., Laporte, N., Mawatari, K., et al., 2018, Nature 557(7705): 392.

Hoag, A., Bradač, M., Brammer, G., et al., 2018, The Astrophysical Journal 854(1): 39.

Karachentsev, I.D., \& Kashibadze, O.G., 2006, Astrophysics 49(1): 3.

Kawka, S., 2010, Moment de casimir: Effet du vide quantique sur l'impulsion d'un milieu bianisotrope. in Physique, (Université de Grenoble, France, 119).

Kent, S.M., \& Gunn, J.E., 1982, The Astronomical Journal 87(7): 945.

Kirilova, D., \& Panayotova, M., 2015, Advances in Astronomy 2015: 10.

Kiselev, V.V., 2003, Acceleration parameter of the Universe expansion and cosmological equation of state, (CERN, Switzerland, 20).

Klimchitskaya, G.L., Mohideen, U., \& Mostepanenko, V.M., 2009, Reviews of Modern Physics 81(4): 1827.

Kłusek-Gawenda, M., Lebiedowicz, P., \& Szczurek, A., 2016, Physical Review C 93(4): 1. 
Kraan-Korteweg, R.C., \& Lahav, O., 1998, Scientific American 279(4): 50.

Lake, G., 1989, The Astrophysical Journal 345: L17.

Langevin, P., 1913, Journal de Physique Théorique et Appliquée 3(1): 553.

Lima, J.A.S., \& Trodden, M., 1996, Physical Review D 53(8): 4280.

Lupi, A., Colpi, M., Devecchi, B., Galanti, G., \& Volonteri, M., 2014, Monthly Notices of the Royal Astronomical Society 442(4): 3616.

Martig, M., Bournaud, F., \& Teyssier, R., 2018, The Astrophysical Journal Draft.

Martin, J., 2012, Comptes Rendus Physique 13(6): 566.

Mayall, N.U., 1960, Advantages of electronic photography for extragalactic spectroscopy. in Annales d'Astrophysique, (344).

McCoy, C.D., 2014, Preprint.

Merritt, D., 1987, The Astrophysical Journal 313: 121.

Natarajan, P., Pacucci, F., Ferrara, A., et al., 2017, The Astrophysical Journal 838(2): 117.

North, P., \& Observatoire de Sauverny, 2011, ASTROPHYSIQUE III: Dynamique stellaire et galactique. (École Polytechnique Fédérale de Lausanne, Lausanne).

Omer Jr, G.C., Page, T.L., \& Wilson, A.G., 1965, The Astronomical Journal 70: 440.

Padmanabhan, T., 2003, Physics Reports 380(5): 235.

Peebles, P.J.E., 1970, The Astronomical Journal 75: 13.

Peñarrubia, J., Ma, Y.-Z., Walker, M.G., \& McConnachie, A., 2014, Monthly Notices of the Royal Astronomical Society 443(3): 2204.

Pence, W.D., 1981, The Astronomical Journal 247(1): 473.

Petrosian, V., 1974, Confrontation of Lemaitre Models and the Cosmological Constant with Observations. in Confrontation of Cosmological Theories with Observational Data, ed M. S. Longair (Springer Netherlands, Dordrecht, 31).

Planck Collaboration, Ade, P.A.R., Aghanim, N., et al., 2016, A\&A 594: A13.

Planck Collaboration, Aghanim, N., Akrami, Y., et al., 2018, Astronomy \& Astrophysics Manuscript.

Randriamampandry, T.H., \& Carignan, C., 2014, Monthly Notices of the Royal Astronomical Society 439(2): 2132.

Richard, J., Kneib, J.-P., Ebeling, H., et al., 2011, Monthly Notices of the Royal Astronomical Society: Letters 414(1): L31.

Riess, A.G., Filippenko, A.V., Challis, P., et al., 1998, The Astronomical Journal 116(3): 1009.

Rood, H.J., Page, T.L., Kintner, E.C., \& King, I.R., 1972, The Astrophysical Journal 175: 627.

Salmon, B., Coe, D., Bradley, L., et al., 2018, The Astrophysical Journal Letters 864(1): L22.

Schuberth, Y., Richtler, T., Dirsch, B., et al., 2006, A\&A 459(2): 391.

Stodolsky, L., 1988, Physics Letters B 201(3): 353.

Van Albada, G.B., 1961, The Astronomical Journal 66: 590.

Van Dokkum, P., Abraham, R., Brodie, J., et al., 2016, The Astrophysical Journal Letters 828(1): L6.

Wang, T., Elbaz, D., Daddi, E., et al., 2016, The Astrophysical Journal 828(1): 56.

White, S.D.M., Navarro, J.F., Evrard, A.E., \& Frenk, C.S., 1993, nature 366(6454): 429.

Xinyu, D., Michael, E.A., Joel, N.B., \& Jon, M.M., 2012, The Astrophysical Journal 755(2): 107.

Zhou, Y., Zhao, Z.-C., \& Chang, Z., 2017, The Astrophysical Journal 847(2): 86.

Zwicky, F., 1937, The Astrophysical Journal 86(3): 217. 